\title{
MATHEMATICAL ANALYSIS OF MULTI-SCALE MODELS OF COMPLEX FLUIDS*
}

\author{
TIEJUN LI $^{\dagger}$ AND PINGWEN ZHANG ${ }^{\ddagger}$
}

\begin{abstract}
The state of the art of the mathematical and numerical analysis of multi-scale models of complex fluids is reviewed. Issues addressed include well-posedness of the models, convergence analysis of the numerical methods, and the structure of stationary solutions of the Doi-Onsager equation.
\end{abstract}

Key words. Complex fluids, multiscale models, well-posedness, numerical analysis, Doi-Onsager equation, micro-macro models

AMS subject classifications. 65C30, 76M35, 82D60, 60H35, 37N10

\section{Introduction}

A polymer is a large molecule made up of many small and simple chemical units, joined together by chemical bonds. For example, polyethylene is a long chainlike molecule composed of ethylene molecules, and DNAs are extremely long chain molecules consists of up to $10^{9}$ nucleotides. Materials composed of macromolecules can display completely different properties other than those composed of small molecules. These polymeric materials are typically very flexible, like rubber, and usually can be easily processed into fibres, thin films, etc. In order to understand these particular properties, we are faced with an issue on how to deal with a large assemble of molecules or monomers. Thus, statistical mechanics is proved to be a very necessary tool even for one single isolated polymer. One approach to investigate one single polymer is to place it in a dilute solution such that interactions between polymers are negligible. As the concentration of the solution increases, the polymers start to overlap and entangle with each other. The limiting state of a polymer solution as concentration increases is known as the polymer melt, which is a state composed only of polymers. Polymer melts have significant industrial applications since most polymeric materials are in this melting state when they are processed into various plastic products. In addition, two or more components of different polymers are usually blended together, and it is important to understand whether and how the polymers mix or separate into different phases.

Polymeric structures in melts, blends and solutions can range from nanometer to microns, millimeters and larger scales. The corresponding time scales of dynamic processes associated with different material properties span an even wide range, from femtoseconds to milliseconds or seconds, even hours for large scale ordering processes such as phase separation in blends. No single model or simulation algorithm can fit such a wide range of length and time scales. Therefore one of the important problems in computational polymer dynamics is multiscale simulation: the bridging of different

*Received: September 29, 2006; accepted (in revised version): December 21, 2006. Communicated by Shi Jin.

${ }^{\dagger}$ LMAM and School of Mathematical Sciences, Peking University, Beijing 100871, P. R. China (tieli@pku.edu.cn).

${ }^{\ddagger}$ LMAM and School of Mathematical Sciences, Peking University, Beijing 100871, P. R. China (pzhang@pku.edu.cn). 
scales to predict macroscopic properties and behavior from fundamental molecular processes.

Polymers are often in liquid phase such as solvated states or melts. This leads to complex and very interesting behavior different from common viscous fluids, for instance, abnormal visco-elasticity. In complex fluids, we can observe phenomena such as shear thinning, rod climbing, extrude swell and tubeless siphon etc [4]. For rigid polymers, when the concentration of the polymers is higher than a critical value $\phi_{c}$ or the environmental temperature is lower than a critical $T_{c}$, we can observe the transition from isotropic phase to aligned phase, i.e. the various liquid crystal phases [13]. In polymer blends or block copolymers, phase separation [80] can occur. How to understand these abundant phenomena provides many research topics for theoretical physicists and applied mathematicians.

The modelling of polymers has a long history. Its modern development is connected with the names like S. F. Edwards, P. J. Flory, P. G. de Gennes, L. Onsager, M. Doi and many, many others. Many classical and excellent textbooks are now available $[25,26,12,13,4,5,14,15,44,24,72]$.

Recently more and more mathematicians are involved in the field of complex fluids. A publication list $[75,76,29,28,82,54,18,48,37,38,84,85,51,11,83,52,7$, $8,9,36,88]$, though incomplete, shows the increased popularity. This field is just at the very beginning, and a lot of problems are still left for mathematicians to explore.

Mathematically speaking, the dynamics of a polymer-solvent system may be modelled by a momentum transport equation for macroscopic momentum

$$
\begin{aligned}
\partial_{t} \boldsymbol{u}+\left(\boldsymbol{u} \cdot \nabla_{\boldsymbol{x}}\right) \boldsymbol{u}+\nabla_{\boldsymbol{x}} p & =\nabla_{\boldsymbol{x}} \cdot \tau, \\
\nabla_{\boldsymbol{x}} \cdot \boldsymbol{u} & =0,
\end{aligned}
$$

where $\boldsymbol{u}$ is the velocity field, $p$ is the pressure, and the stress $\tau=\tau_{s}+\tau_{p} . \tau_{s}$ is the stress due to the solvent, which can be modeled by $\tau_{s}=\eta_{s} D, D=1 / 2\left(\nabla_{\boldsymbol{x}} \boldsymbol{u}+\nabla_{\boldsymbol{x}} \boldsymbol{u}^{T}\right)$, where $\eta_{s}$ is the viscosity coefficient of the solvent. $\tau_{p}$ describes the polymer contribution to stress. It is given either by a complex constitutive equation, or through ensemble average over the configurations of the polymers by kinetic theory.

The traditional macroscopic approach to describe $\tau_{p}$ is usually through some empirical constitutive relations to express the viscoelastic properties $[4,74]$. There are different extensions of Newtonian constitutive relations in this regard. One typical extension is the so-called generalized Newtonian fluid

$$
\lambda \stackrel{\nabla}{\tau}_{p}+\tau_{p}+f\left(\tau_{p}, D\right)=\eta D
$$

where

$$
\underset{\tau_{p}}{\nabla}=\frac{\partial \tau_{p}}{\partial t}+\left(\boldsymbol{u} \cdot \nabla_{\boldsymbol{x}}\right) \tau_{p}-\kappa \cdot \tau_{p}-\tau_{p} \cdot \kappa^{T}
$$

is the upper convective derivative [4], $\lambda$ is a relaxation time, and $\kappa=\left(\nabla_{\boldsymbol{x}} \boldsymbol{u}\right)^{T}$. Here $\tau_{p}$ depends on the strain rate through a differential equation. The well-known differential models for $\tau_{p}$ are such as the Oldroyd-B model $f\left(\tau_{p}, D\right)=0$, Johnson-Segalman model $f\left(\tau_{p}, D\right)=\alpha \lambda\left(\tau_{p} \cdot D+D \cdot \tau_{p}\right)$ etc. Another simple empirical constitutive equation is

$$
\tau_{p}=\eta(D) D
$$

Here $\eta(\cdot)$ is a function of $D$ instead of a constant in Newtonian fluid, which means the viscosity coefficient depends on the strain rate tensor itself. Another typical extension 
is called integral models, for example the Maxwell model, in which

$$
\tau_{p}=\int_{-\infty}^{t} \frac{\eta_{s}}{\lambda} \exp \left(-\frac{t-s}{\lambda}\right) \dot{\gamma}(s) d s,
$$

where $\dot{\gamma}=\frac{1}{2}\left(\kappa+\kappa^{T}\right) . \frac{\eta_{s}}{\lambda} \exp \left(-\frac{t-s}{\lambda}\right)$ is called the relaxation modulus representing the memory effect in viscoelastic fluid.

Though macroscopic empirical models achieve some success, it is quite phenomenological. To predict the macroscopic behavior of material from the microscopic description is the modern viewpoint of multiscale modelling. Some pioneers have tried to develop the force field for molecular dynamical simulations of polymers from quantum mechanics, and there has been systematic study for fluids with molecular dynamics $[27,1]$. But as is well-known, molecular dynamics is very limited for its length and time scale. In order to investigate larger scale information, kinetic theory is applied. In kinetic theory, each polymer is coarse grained as a bead-spring chain or rigid rod (c.f. section 2). A stochastic differential equation for the connecting vector $\boldsymbol{Q}$ of springs or the orientation vector $\boldsymbol{m}$ of rods is thus needed, or equivalently the Fokker-Planck equation for probability density function $\psi$ is involved. Some expression for the polymeric stress relates the equation (1.1) with the kinetic equations. To discuss the well-posedness and numerical analysis of different kinds of systems will be the main task of this review. As will be seen later, the analysis known up-todate is only confined to the dumbbell model and the rod-like model. The analysis for other mathematical models in complex fluids, such as SCFT (self consistent field theory) [77], Flory-Huggins variational problem [15], Ginzburg-Landau equation [80], Reptation model [72], are almost unexplored!

\section{Derivation of the mathematical models}

Most polymers consist of long chains or branches of repeated chemical units, such as BPA-PC, Polyolefin etc. The full description of each atom in the polymer by molecular dynamics is not feasible for the huge computational effort and its limited timescale. Coarse grained models are necessary to be developed for considering macroscopic space and time properties of complex fluids. Typical models such as the bead-spring chain for flexible polymers and the rigid rod model for liquid crystalline polymers have been established by some pioneers in polymer science. The basic dynamical theory for these models are collected in the classic books by Bird et al. $[4,5]$ and Doi and Edwards [14]. From the bending rigidity and typical size of each polymer, the coarse grained models can be classified into three classes: the flexible, semi-flexible, and rigid molecules.

There are two important length scales. One is the bending persistence length of a polymer $l_{p}$, which is defined to be the correlation length for the tangent vector at different places in the polymer. The other is the length of the polymer $l$. According to the relative size of $l$ and $l_{p}$, polymers are classified as

\begin{tabular}{ccc} 
flexible & semi-flexible & rigid \\
\hline$l \gg l_{p}$ & $\quad l \sim O\left(l_{p}\right)$ & $l \ll l_{p}$
\end{tabular}

For the flexible polymers, the schematic representation is the dumbbell model, which is a long bead-spring chain where each spring between two beads represents a connecting vector between two segments in the polymer whose distance is much longer than $l_{p}$ (Figure 2.1). A suitable stretching potential may be introduced for 

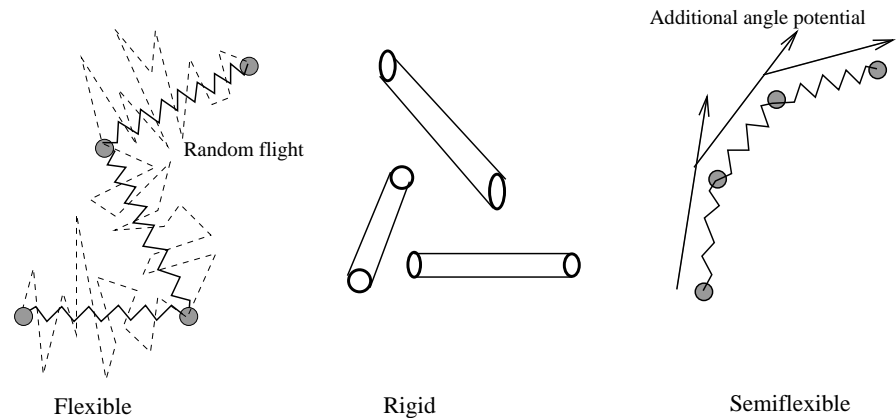

FIG. 2.1. Sketch of flexible, semi-flexible and rigid polymers.

each spring. After ignoring the excluded volume interaction, the central limit theorem and the loss of correlation between the connecting vectors suggests the random flight Gaussian chain is a good approximation. For the rigid polymers $\left(l \ll l_{p}\right)$, they are often modelled as rigid rods which cannot be bent. This type of model is very successful for liquid crystalline polymers. For the semi-flexible polymers, they can also be thought of as a bead-spring chain system, but the correlation between the end-to-end vectors will lead to an additional potential for the bending angles. In general, for simplification of analysis, this potential is taken as a quadratic form, and the spring potential are also chosen as a quadratic form. This type of model is used for biological macromolecules, such as DNA, etc.[87]. We will only consider the flexible and rigid rod case in this review. The main derivation procedures are drawn from $[14,5]$.

2.1. Dumbbell model. We only consider two-beads connected by one spring case here. Since each bead is a coarse grained particle representing several chemical units, each bead is in mesoscopic scale. It will experience four kinds of forces in the dilute case where there is no interaction among dumbbells.

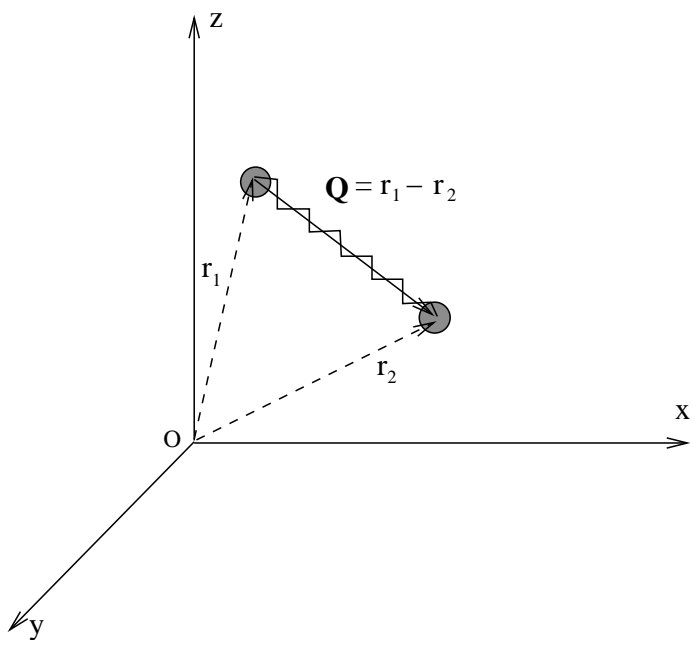

FIG. 2.2. Sketch of the dumbbell model representation. 
A. Inertial force

$$
m_{i} \ddot{\boldsymbol{r}}_{i}, \quad i=1,2,
$$

where the dot means the derivative with respect to time.

B. Frictional force

$$
-\zeta_{i}\left(\dot{\boldsymbol{r}}_{i}-\boldsymbol{u}\left(\boldsymbol{r}_{i}\right)\right), \quad i=1,2
$$

where $\zeta_{i}=6 \pi \eta_{s} a_{i}$ by Stokes' formula, which is the friction coefficient for the $i$-th bead. $a_{i}$ is the radius of the $i$-th bead.

C. Spring force

$$
\boldsymbol{F}_{i}=-\nabla_{\boldsymbol{r}_{i}} \Psi\left(\boldsymbol{r}_{1}, \boldsymbol{r}_{2}\right), \quad i=1,2 .
$$

We have $\boldsymbol{F}_{1}=-\boldsymbol{F}_{2}$ by Newton's Third Law. The choice of the potential $\Psi$ will be discussed later.

D. Brownian force

$$
\boldsymbol{F}_{W_{i}}=\sigma_{i} \dot{\boldsymbol{W}}_{i}, \quad i=1,2,
$$

where $\boldsymbol{W}_{i}$ are independent standard Brownian motions. $\sigma_{i}=\sqrt{2 k_{B} T \zeta_{i}}$ by the fluctuation-dissipation theorem, where $k_{B}$ is the Boltzmann constant, and $T$ is the temperature

From the force balance condition we have the Langevin equation for two beads:

$$
\begin{aligned}
& m_{1} \ddot{\boldsymbol{r}}_{1}=-\zeta_{1}\left(\dot{\boldsymbol{r}}_{1}-\boldsymbol{u}\left(\boldsymbol{r}_{1}\right)\right)+\boldsymbol{F}_{1}+\sigma_{1} \dot{\boldsymbol{W}}_{1}, \\
& m_{2} \ddot{\boldsymbol{r}_{1}}=-\zeta_{2}\left(\dot{\boldsymbol{r}}_{2}-\boldsymbol{u}\left(\boldsymbol{r}_{2}\right)\right)+\boldsymbol{F}_{2}+\sigma_{2} \dot{\boldsymbol{W}}_{2} .
\end{aligned}
$$

In the high friction regime $\left(\zeta_{i} \gg 1\right)$, we can adopt the overdamped Brownian dynamics approximation [71]:

$$
\begin{aligned}
\zeta_{1}\left(\dot{\boldsymbol{r}}_{1}-\boldsymbol{u}\left(\boldsymbol{r}_{1}\right)\right) & =\boldsymbol{F}_{1}+\sigma_{1} \dot{\boldsymbol{W}}_{1}, \\
\zeta_{2}\left(\dot{\boldsymbol{r}}_{2}-\boldsymbol{u}\left(\boldsymbol{r}_{2}\right)\right) & =\boldsymbol{F}_{2}+\sigma_{2} \dot{\boldsymbol{W}}_{2} .
\end{aligned}
$$

Thus we have an equation for the center of resistance,

$$
\mathbb{E} \boldsymbol{r}_{c}=\frac{\zeta_{1} \boldsymbol{u}\left(\boldsymbol{r}_{1}\right)+\zeta_{2} \boldsymbol{u}\left(\boldsymbol{r}_{2}\right)}{\zeta_{1}+\zeta_{2}} \approx \boldsymbol{u}\left(\boldsymbol{r}_{c}\right)
$$

where

$$
\boldsymbol{r}_{c}=\frac{\zeta_{1} \boldsymbol{r}_{1}+\zeta_{2} \boldsymbol{r}_{2}}{\zeta_{1}+\zeta_{2}}=\frac{1}{2}\left(\boldsymbol{r}_{1}+\boldsymbol{r}_{2}\right)
$$

under the assumption $\zeta_{1}=\zeta_{2}=\zeta$. The approximation in (2.5) utilizes the equation $\boldsymbol{u}(\boldsymbol{r}) \approx \boldsymbol{u}\left(\boldsymbol{r}_{c}\right)+\kappa \cdot\left(\boldsymbol{r}-\boldsymbol{r}_{c}\right)$, where $\kappa=\left(\nabla_{\boldsymbol{x}} \boldsymbol{u}\right)^{T}$ is the strain rate.

Define the end-to-end vector $\boldsymbol{Q}=\boldsymbol{r}_{2}-\boldsymbol{r}_{1}$, it satisfies the equation

$$
\dot{\boldsymbol{Q}}=\kappa \cdot \boldsymbol{Q}-\frac{2}{\zeta} \boldsymbol{F}_{1}+\sqrt{\frac{2 k_{B} T}{\zeta}}\left(\dot{\boldsymbol{W}}_{2}-\dot{\boldsymbol{W}}_{1}\right)
$$


Noticing that $\frac{1}{\sqrt{2}}\left(\boldsymbol{W}_{2}-\boldsymbol{W}_{1}\right)$ is also a standard Brownian motion, we have

$$
\dot{\boldsymbol{Q}}=\kappa \cdot \boldsymbol{Q}-\frac{2}{\zeta} \boldsymbol{F}_{1}+\sqrt{\frac{4 k_{B} T}{\zeta}} \dot{\boldsymbol{W}}
$$

Thus we obtain the stochastic differential equations (SDE) for the two-beads spring system is

$$
\begin{aligned}
\dot{\boldsymbol{r}}_{c} & =\boldsymbol{u}\left(\boldsymbol{r}_{c}\right) \\
\dot{\boldsymbol{Q}} & =\kappa \cdot \boldsymbol{Q}-\frac{2}{\zeta} \boldsymbol{F}(\boldsymbol{Q})+\sqrt{\frac{4 k_{B} T}{\zeta}} \dot{\boldsymbol{W}} .
\end{aligned}
$$

Note that we have made an ad-hoc approximation in (2.5).

Now we consider the choice of spring potential. Two choices are commonly used:

$$
\text { Force } \boldsymbol{F}(\boldsymbol{Q}) \quad \text { Potential } \Psi(\boldsymbol{Q})
$$

\begin{tabular}{lcc}
\hline Hookean spring & $H \boldsymbol{Q}$ & $\frac{1}{2} H Q^{2}$ \\
FENE spring & $\frac{H \boldsymbol{Q}}{1-\left(Q / Q_{0}\right)^{2}}$ & $-\frac{1}{2} H Q_{0}^{2} \ln \left(1-\left(Q / Q_{0}\right)^{2}\right)$
\end{tabular}

where FENE means "Finitely Extendable Nonlinear Elastic", $H$ is the elastic spring constant, and $Q_{0}$ is the maximal extension of the FENE spring.

From stochastic analysis $[72,69]$ the probability density function (pdf) $\psi(\boldsymbol{x}, \boldsymbol{Q}, t)$ satisfies the Fokker-Planck equations, which is usually called the Smoluchowski equations in the polymer science,

$$
\partial_{t} \psi+\nabla_{\boldsymbol{x}} \cdot(\boldsymbol{u} \psi)+\nabla_{\boldsymbol{Q}} \cdot\left[\left(\kappa \boldsymbol{Q}-\frac{2}{\zeta} \boldsymbol{F}\right) \psi\right]=\frac{2 k_{B} T}{\zeta} \Delta_{\boldsymbol{Q}} \psi
$$

After knowing the dynamical equation for the dumbbells, the next step is to understand the contribution of these dumbbells to the macroscopic flow. This is described by the polymeric stress tensor $\tau_{p}$ in (1.1), which comes from two aspects:

1. An arbitrary plane in the suspension may at any moment be straddled by the two beads of the dumbbell, and there will be a force of tension or compression transmitted though the connector. This part will be labelled as $\tau_{p}^{(c)}$.

2. The beads themselves may cross the arbitrary plane and bring with them a certain amount of momentum. This part will be labelled as $\tau_{p}^{(b)}$.

We will assume that the number density of polymers is $n$, and the stress tensor $\tau_{p}=\tau_{p}^{(c)}+\tau_{p}^{(b)}$. Each term is obtained as follows [5]:

A. Expression for $\tau_{p}^{(c)}$ :

Let us consider a cube of volume $\frac{1}{n}$, one of whose faces with orientation given by a unit normal vector $\boldsymbol{n}$. In general, there will be only one dumbbell in the cube. If we toss one dumbbell with orientation $Q$ into this cubical region, the probability that the dumbbell cuts the shaded plane is $(\boldsymbol{Q} \cdot \boldsymbol{n}) /(1 / n)^{1 / 3}$. The force transmitted by the connector is $\boldsymbol{F}^{(c)}(\boldsymbol{Q})$. Taking the expectation of the force we obtain

$$
\int_{\mathbb{R}^{3}} \frac{\boldsymbol{Q} \cdot \boldsymbol{n}}{(1 / n)^{1 / 3}} \boldsymbol{F}^{(c)}(\boldsymbol{Q}) \psi(\boldsymbol{Q}, t) d \boldsymbol{Q}
$$




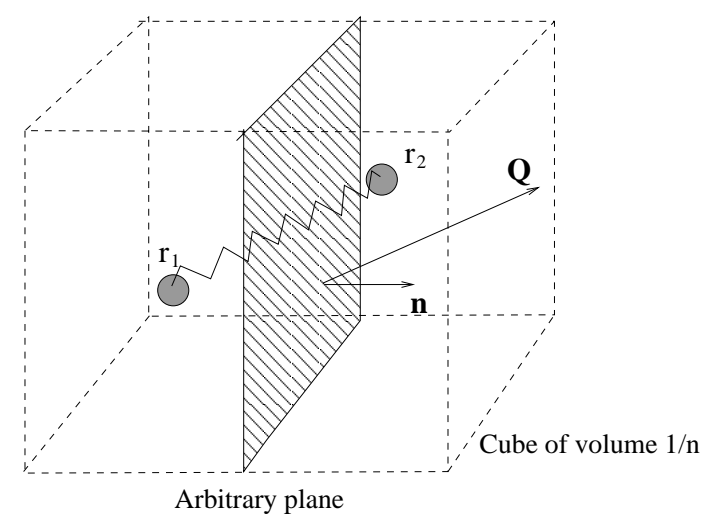

FIG. 2.3. Sketch of the derivation of stress $\tau_{p}^{(c)}$ which comes from the connector $\boldsymbol{Q}$ intersecting with the control surface.

The force per area is $n\left\langle\boldsymbol{Q} \cdot \boldsymbol{n} \boldsymbol{F}^{(c)}(\boldsymbol{Q})\right\rangle=\boldsymbol{n} \cdot \tau_{p}^{(c)}$, thus the stress is

$$
\tau_{p}^{(c)}=n\left\langle\boldsymbol{Q} \otimes \boldsymbol{F}^{(c)}(\boldsymbol{Q})\right\rangle,
$$

where $\langle\cdot\rangle$ means the ensemble average in $\boldsymbol{Q}$ space.

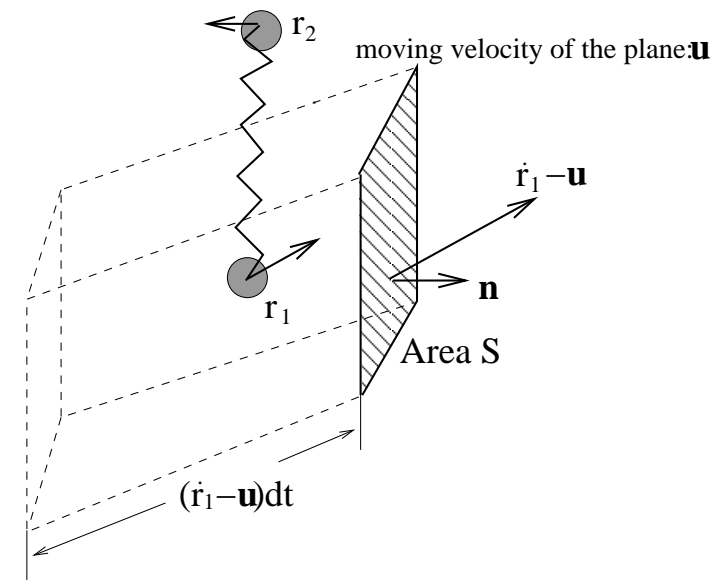

FIG. 2.4. Sketch of the derivation of stress $\tau_{p}^{(b)}$ which comes from the transportation of the momentum by the dumbbells.

B. Expression for $\tau_{p}^{(b)}$ :

Consider a moving plane with velocity $\boldsymbol{u}$, the number of bead 1 with velocity $\dot{\boldsymbol{r}}_{1}$ crossing the surface with area $S$ per unit time will be $-n\left(\dot{\boldsymbol{r}_{1}}-\boldsymbol{u}\right) \cdot \boldsymbol{n} S$, and correspondingly the momentum transported is

$$
-n\left(\left(\dot{\boldsymbol{r}_{1}}-\boldsymbol{u}\right) \cdot \boldsymbol{n} S\right) m_{1}\left(\dot{\boldsymbol{r}}_{1}-\boldsymbol{u}\right) .
$$

A similar procedure may be taken for bead 2, and the mean transported momentum will be

$$
-\iint \sum_{i=1}^{2} n\left(\left(\dot{\boldsymbol{r}_{i}}-\boldsymbol{u}\right) \cdot \boldsymbol{n} S\right) m_{i}\left(\dot{\boldsymbol{r}_{i}}-\boldsymbol{u}\right) \Psi\left(\dot{\boldsymbol{r}_{1}}, \dot{\boldsymbol{r}_{2}}\right) d \dot{\boldsymbol{r}_{1}} d \dot{\boldsymbol{r}_{2}}
$$


At equilibrium, the Maxwellian is assumed for $\Psi\left(\dot{\boldsymbol{r}_{1}}, \dot{\boldsymbol{r}_{2}}\right)$,

$$
\Psi\left(\dot{\boldsymbol{r}_{1}}, \dot{\boldsymbol{r}_{2}}\right)=\frac{1}{Z} \exp \left(-\left(m_{1}\left(\dot{\boldsymbol{r}_{1}}-\boldsymbol{u}\right)^{2}+m_{2}\left(\dot{\boldsymbol{r}_{2}}-\boldsymbol{u}\right)^{2}\right) / 2 k_{B} T\right)
$$

where $Z=\iint \exp \left(-\left(m_{1}\left(\dot{\boldsymbol{r}_{1}}-\boldsymbol{u}\right)^{2}+m_{2}\left(\dot{\boldsymbol{r}_{2}}-\boldsymbol{u}\right)^{2}\right) / 2 k_{B} T\right) d \dot{\boldsymbol{r}_{1}} d \dot{\boldsymbol{r}_{2}}$. It is not difficult to show that the mean transported momentum equals $-2 n k_{B} T \boldsymbol{I} \cdot \boldsymbol{n} S=S \boldsymbol{n} \cdot \tau_{p}^{(b)}$, and hence $\tau_{p}^{(b)}=-2 n k_{B} T \boldsymbol{I}$. At equilibrium $-n k_{B} T \boldsymbol{I}$ will contribute to the pressure, and thus we have

$$
\tau_{p}^{(b)}=-n k_{B} T \boldsymbol{I} .
$$

Combining (2.13) and (2.17) together, we get the Kramers' expression of the polymeric stress for the dumbbell model,

$$
\tau_{p}=-n k_{B} T \boldsymbol{I}+n\langle\boldsymbol{F}(\boldsymbol{Q}) \otimes \boldsymbol{Q}\rangle .
$$

It is often convenient to drop the term $-n k_{B} T \boldsymbol{I}$ because it does not contribute to the stress after differentiation.

Standard scaling analysis will introduce the non-dimensional parameters:

$$
D e=\frac{T_{r}}{T_{c}}, \quad R e=\frac{\rho U_{0} L_{0}}{\eta}, \quad \gamma=\frac{\eta_{s}}{\eta},
$$

where $T_{r}=\zeta /(4 H)$ is the mesoscopic relaxation time scale of the spring, $T_{c}=L_{0} / U_{0}$ is the macroscopic convective time scale, $\rho$ is the density and $\eta$ is the total viscosity of the fluid. De is called Deborah number, which is the most important parameter in non-Newtonian fluids. Re and $\gamma$ are the Reynolds number and viscosity ratio, respectively.

The non-dimensionalized system becomes:

$$
\begin{aligned}
\partial_{t} \boldsymbol{u}+\left(\boldsymbol{u} \cdot \nabla_{\boldsymbol{x}}\right) \boldsymbol{u}+\nabla_{\boldsymbol{x}} p & =\frac{\gamma}{R e} \Delta_{\boldsymbol{x}} \boldsymbol{u}+\frac{1-\gamma}{R e D e} \nabla_{\boldsymbol{x}} \cdot \tau_{p}, \\
\nabla_{\boldsymbol{x}} \cdot \boldsymbol{u} & =0, \\
\tau_{p} & =\langle\boldsymbol{F}(\boldsymbol{Q}) \otimes \boldsymbol{Q}\rangle, \\
\frac{d \boldsymbol{x}}{d t} & =\boldsymbol{u}(\boldsymbol{x}), \\
\frac{d \boldsymbol{Q}}{d t} & =\kappa \cdot \boldsymbol{Q}-\frac{1}{2 D e} \boldsymbol{F}(\boldsymbol{Q})+\frac{1}{\sqrt{D e}} \dot{\boldsymbol{W}}(t) .
\end{aligned}
$$

The equations (2.23) and (2.24) can also be written in Eulerian form, which forms a stochastic PDE

$$
\partial_{t} \boldsymbol{Q}+\left(\boldsymbol{u} \cdot \nabla_{\boldsymbol{x}}\right) \boldsymbol{Q}=\kappa \cdot \boldsymbol{Q}-\frac{1}{2 D e} \boldsymbol{F}(\boldsymbol{Q})+\frac{1}{\sqrt{D e}} \dot{\boldsymbol{W}}(t),
$$

and the Smoluchowski equation is:

$$
\partial_{t} \psi+\nabla_{\boldsymbol{x}} \cdot(\boldsymbol{u} \psi)+\nabla_{\boldsymbol{Q}} \cdot\left[\left(\kappa \boldsymbol{Q}-\frac{1}{2 D e} \boldsymbol{F}(\boldsymbol{Q})\right) \psi\right]=\frac{1}{2 D e} \Delta_{\boldsymbol{Q}} \psi .
$$

Recently, Barret and Süli [3] considered a more refined formulation for the dumbbell model, the main derivation procedure could be found in the paper [3]. They take 
less approximations in deriving equations (2.9) and (2.10). Formally, the SDEs of their model in non-dimensionalized form are

$$
\begin{aligned}
\frac{d \boldsymbol{x}}{d t} & =\boldsymbol{u}(\boldsymbol{x})+\frac{\varepsilon}{2 \sqrt{D e}} \dot{\boldsymbol{W}}^{\boldsymbol{x}}(t), \\
\frac{d \boldsymbol{Q}}{d t} & =\left(\nabla_{\boldsymbol{x}} \mathcal{A}_{\varepsilon}^{\boldsymbol{x}} \boldsymbol{u}\right)^{T} \cdot \boldsymbol{Q}-\frac{1}{2 D e} \boldsymbol{F}(\boldsymbol{Q})+\frac{1}{\sqrt{D e}} \dot{\boldsymbol{W}}^{\boldsymbol{Q}}(t),
\end{aligned}
$$

where $\varepsilon=\sqrt{\frac{k_{B} T}{H}} / L_{0}$ is the non-dimensionalized micro-macro size ratio. $\boldsymbol{W}^{\boldsymbol{x}}(t)$ and $\boldsymbol{W}^{\boldsymbol{Q}}(t)$ are independent standard Wiener process with respect to space variable $\boldsymbol{x}$ and configuration variable $\boldsymbol{Q}$. The mollifier operator $\mathcal{A}_{\varepsilon}^{x}$ is defined as

$$
\mathcal{A}_{\varepsilon}^{\boldsymbol{x}} \boldsymbol{u}=\frac{1}{\varepsilon} \int_{-\frac{\varepsilon}{2}}^{\frac{\varepsilon}{2}} \boldsymbol{u}(\boldsymbol{x}+\mu \boldsymbol{Q}) d \mu .
$$

Correspondingly, the equation for $\psi$ takes the form

$$
\partial_{t} \psi+\nabla_{\boldsymbol{x}} \cdot(\boldsymbol{u} \psi)+\nabla_{\boldsymbol{Q}} \cdot\left[\left(\left(\nabla_{\boldsymbol{x}} \mathcal{A}_{\varepsilon}^{\boldsymbol{x}} \boldsymbol{u}\right)^{T} \cdot \boldsymbol{Q}-\frac{1}{2 D e} \boldsymbol{F}(\boldsymbol{Q})\right) \psi\right]=\frac{1}{2 D e} \Delta_{\boldsymbol{Q}} \psi+\frac{\varepsilon^{2}}{8 D e} \Delta_{\boldsymbol{x}} \psi
$$

Though $\varepsilon$ is very small with the order about $10^{-4}$, theoretically the nature of Smoluchowski equation is totally changed. The polymeric stress $\tau_{p}$ is also changed by replacing $\psi$ with a mollified $\psi$. Barret and Süli take advantage of these properties to perform well-posedness analysis.

2.2. Rod-like model. For the description of a liquid crystal, it is more convenient to describe the conformation of rigid rods by its pdf $\psi(\boldsymbol{x}, \boldsymbol{m}, t)$. Here $\boldsymbol{x}$ is the center of mass of the rod, $|\boldsymbol{m}|=1$ is a unit vector in the spherical surface $\mathbb{S}^{2}$ which represents the orientation of the rod. The pdf has the properties $\psi(\boldsymbol{x}, \boldsymbol{m}, t) \geq 0$ and $\int_{\mathbb{R}^{3}} \int_{\mathbb{S}^{2}} \psi(\boldsymbol{x}, \boldsymbol{m}, t) d \boldsymbol{m} d \boldsymbol{x}=1$. We will only consider the homogeneous case here, i.e., the positional distribution is uniform or the concentration gradient is 0 , namely

$$
\nabla_{\boldsymbol{x}} \int_{\mathbb{S}^{2}} \psi(\boldsymbol{x}, \boldsymbol{m}, t) d \boldsymbol{m}=0 .
$$

With this assumption, we can simplify the normalization condition to

$$
\int_{\mathbb{S}^{2}} \psi(\boldsymbol{x}, \boldsymbol{m}, t) d \boldsymbol{m}=1 .
$$

In general, the free energy of the homogeneous system can be expressed as

$$
\mathcal{F}[\psi]=n \int_{\mathbb{S}^{2}}\left(k_{B} T \psi \ln \psi+U \psi\right) d \boldsymbol{m} .
$$

Here $n$ is the number density of polymers, $U$ is an external field or a mean field interaction potential for polymers in the concentrated case which will be described more clearly later. (If $U$ is a mean field potential, there would be a coefficient $1 / 2$ before the potential energy term. The difference would appear when taking variation with respect to $\psi$.)

From the continuity equation of probability density

$$
\partial_{t} \psi=-\nabla_{\boldsymbol{m}} \cdot(\boldsymbol{v} \psi),
$$




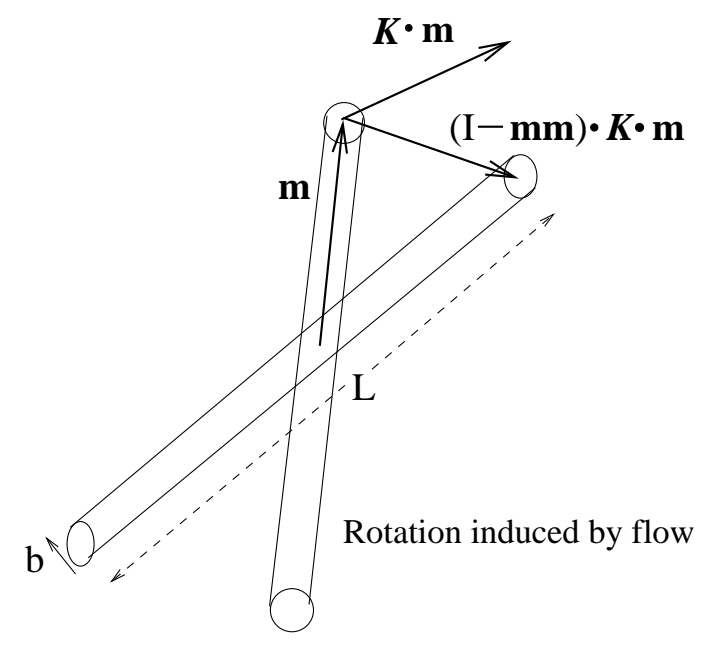

FIG. 2.5. Sketch of the rotational diffusion of the rods.

where $\boldsymbol{v}$ is the velocity of the rod in $\boldsymbol{m}$ space. For the rotation of the rod,

$$
\boldsymbol{v}=\omega \times \boldsymbol{m},
$$

where $\omega$ is the angular velocity. It comes from three aspects.

A. Angular velocity induced by macroscopic velocity gradient:

$$
\omega_{1}=\boldsymbol{m} \times \dot{\boldsymbol{m}}=\boldsymbol{m} \times[(\boldsymbol{I}-\boldsymbol{m} \otimes \boldsymbol{m}) \cdot \kappa \cdot \boldsymbol{m}],
$$

where $\dot{\boldsymbol{m}}$ is the projection of velocity difference for the two ends of the rod to $\boldsymbol{m}$ direction. $\kappa \cdot \boldsymbol{m} \approx \boldsymbol{u}\left(\boldsymbol{x}_{2}\right)-\boldsymbol{u}\left(\boldsymbol{x}_{1}\right)$. An approximation similar to that in equation (2.5) is taken.

B. Angular velocity induced by the external field: (a linear response system is assumed)

$$
\omega_{2}=\boldsymbol{m} \times\left[(\boldsymbol{I}-\boldsymbol{m} \otimes \boldsymbol{m}) \cdot\left(-\frac{1}{\zeta_{r}} \nabla_{\boldsymbol{m}} U\right)\right],
$$

where $\zeta_{r}$ is the rotational friction coefficient, and may be deduced by Stokes' formula combined with the Shish-Kebab model or more delicate calculations [15]. A common choice for $\zeta_{r}$ is

$$
\zeta_{r}=\frac{\pi \eta_{s} L^{3}}{3\left(\ln \frac{L}{b}-c\right)},
$$

where $L$ is the length of the rod, $b$ is the diameter of the cylinder and $c$ is some constant.

C. Angular velocity induced by Brownian force:

$$
\omega_{3}=\boldsymbol{m} \times\left[(\boldsymbol{I}-\boldsymbol{m} \otimes \boldsymbol{m}) \cdot\left(-\frac{1}{\zeta_{r}} \nabla_{\boldsymbol{m}}\left(k_{B} T \ln \psi\right)\right)\right] .
$$

A simple calculation shows for an arbitrary vector $\boldsymbol{f}$ that

$$
\boldsymbol{m} \times[(\boldsymbol{I}-\boldsymbol{m} \otimes \boldsymbol{m}) \cdot \boldsymbol{f}]=\boldsymbol{m} \times \boldsymbol{f} .
$$


Define the rotational operator $\mathcal{R}=\boldsymbol{m} \times \nabla_{\boldsymbol{m}}$. Then we have

$$
\begin{aligned}
\partial_{t} \psi & =-\nabla_{\boldsymbol{m}} \cdot(\boldsymbol{v} \psi)=-\left(\boldsymbol{m} \times \nabla_{\boldsymbol{m}}\right) \cdot(\omega \psi) \\
& =-\mathcal{R} \cdot(\omega \psi)=-\mathcal{R} \cdot\left(\left(\omega_{1}+\omega_{2}+\omega_{3}\right) \psi\right) \\
& =\mathcal{R} \cdot\left[\frac{1}{\zeta_{r}}\left(k_{B} T \mathcal{R} \psi+\psi \mathcal{R} U\right)\right]-\mathcal{R} \cdot(\boldsymbol{m} \times \kappa \cdot \boldsymbol{m} \psi) \\
& =D_{r} \mathcal{R} \cdot\left[\left(\mathcal{R} \psi+\frac{\psi}{k_{B} T} \mathcal{R} U\right)\right]-\mathcal{R} \cdot(\boldsymbol{m} \times \kappa \cdot \boldsymbol{m} \psi),
\end{aligned}
$$

where $D_{r}=k_{B} T / \zeta_{r}$ is the rotational diffusion coefficient.

We will only consider the concentrated case in this paper, which is the most relevant case in industrial applications. Each rod is regarded as located in a background mean field enforced by surrounding rigid rods. Because each rod takes up some space, it prevents other rods from rotating and moving. This will induce an excluded volume potential, first proposed by L. Onsager in his seminal work [70].

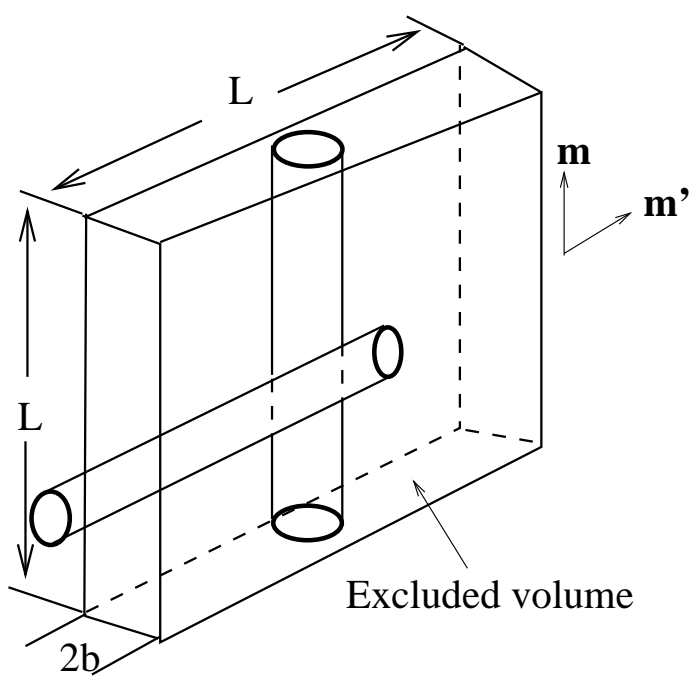

FIG. 2.6. Sketch of the excluded volume potential of the rods

The excluded volume between the rods with the direction $\boldsymbol{m}$ and $\boldsymbol{m}^{\prime}$ can be calculated easily from the Figure 2.6 as

$$
V_{e v}\left(\boldsymbol{m}, \boldsymbol{m}^{\prime}\right)=2 b L^{2}|\sin \theta|=2 b L^{2}\left|\boldsymbol{m} \times \boldsymbol{m}^{\prime}\right|,
$$

so the mean field potential can be taken as

$$
U_{\text {Onsager }}(\boldsymbol{m})=\int_{\mathbb{S}^{2}} V_{e v}\left(\boldsymbol{m}, \boldsymbol{m}^{\prime}\right) \psi\left(\boldsymbol{m}^{\prime}\right) d \boldsymbol{m}^{\prime}=2 b L^{2} \int_{\mathbb{S}^{2}}\left|\boldsymbol{m} \times \boldsymbol{m}^{\prime}\right| \psi\left(\boldsymbol{m}^{\prime}\right) d \boldsymbol{m}^{\prime}
$$

A commonly used approximation is the Maier-Saupe [62] potential which is more convenient for its differentiability

$$
U_{\mathrm{MS}}(\boldsymbol{m})=2 b L^{2} \int_{\mathbb{S}^{2}}\left|\boldsymbol{m} \times \boldsymbol{m}^{\prime}\right|^{2} \psi\left(\boldsymbol{m}^{\prime}\right) d \boldsymbol{m}^{\prime} .
$$


The physical implication of the Maier-Saupe [62] potential is different from that of Onsagers [70]. In the original presentation of Maier-Saupe [62] it was assumed that the intensity is due to van der Waals forces and is temperature-independent. In realistic situations contributions from the steric repulsions may be non-negligible and they do depend on the temperature [13].

As the mesoscopic equation (2.39) for rods has been found, the polymeric stress $\tau_{p}$ can be obtained. For liquid crystal, it is composed of two parts [15],

$$
\tau_{p}=\tau_{p}^{(E)}+\tau_{p}^{(V)}
$$

Here $\tau_{p}^{(E)}$ is the elastic stress which comes from the virtual work principle, and $\tau_{p}^{(V)}$ is the viscous stress which is related to the energy dissipation function.

For $\tau_{p}^{(E)}$, the virtual elastic energy $\delta \mathcal{F}$ induced by the virtual deformation $\delta \epsilon_{\alpha \beta}$ has form $\delta \mathcal{F}=\left(\tau_{p}^{(E)}\right)_{\alpha \beta} \delta \epsilon_{\alpha \beta}$, where Einstein's summation convention is assumed and

$$
\delta \mathcal{F}=n \int_{\mathbb{S}^{2}} d \boldsymbol{m}\left(k_{B} T \delta \psi \ln \psi+k_{B} T \delta \psi+\delta \psi U\right)
$$

by (2.33). The change $\delta \psi$ is obtained from the Smoluchowski equation (2.39). For the instantaneous deformation, the strain $\delta \epsilon=\kappa \delta t$ is dominated. Thus,

$$
\delta \psi=-\mathcal{R} \cdot(\boldsymbol{m} \times \kappa \cdot \boldsymbol{m} \psi) \delta t=-\mathcal{R} \cdot(\boldsymbol{m} \times \delta \epsilon \cdot \boldsymbol{m} \psi),
$$

and so

$$
\delta \mathcal{F}=n \int_{\mathbb{S}^{2}} d \boldsymbol{m}\left(-k_{B} T \mathcal{R} \cdot(\boldsymbol{m} \times \delta \epsilon \cdot \boldsymbol{m})+(\boldsymbol{m} \times \delta \epsilon \cdot \boldsymbol{m}) \cdot \mathcal{R} U\right) \psi
$$

by integrating by parts for the operator $\mathcal{R}$. Direct calculation shows that $\mathcal{R} \cdot(\boldsymbol{m} \times \delta \epsilon$. $\boldsymbol{m})=-3 \delta \epsilon_{\alpha \beta}\left(m_{\alpha} m_{\beta}-\frac{1}{3} \delta_{\alpha \beta}\right)$. Hence

$$
\delta \mathcal{F}=n \delta \epsilon_{\alpha \beta}\left(3 k_{B} T\left\langle m_{\alpha} m_{\beta}-\frac{1}{3} \delta_{\alpha \beta}\right\rangle-\left\langle(\boldsymbol{m} \times \mathcal{R} U)_{\alpha} m_{\beta}\right\rangle\right) .
$$

Define the orientational tensor $\boldsymbol{S}=\left\langle\boldsymbol{m} \otimes \boldsymbol{m}-\frac{1}{3} \boldsymbol{I}\right\rangle$. Then

$$
\tau_{p}^{(E)}=3 n k_{B} T \boldsymbol{S}-n\langle(\boldsymbol{m} \times \mathcal{R} U) \otimes \boldsymbol{m}\rangle .
$$

The viscous stress $\tau_{p}^{(V)}$ is related to the energy dissipation function $W=n \sum_{i}\left\langle\boldsymbol{F}_{i}\right.$. $\left.\boldsymbol{v}_{i}\right\rangle$ by $W=\kappa_{\alpha \beta}\left(\tau_{p}^{(V)}\right)_{\alpha \beta}$. Detailed calculation [15] shows that

$$
W=n \zeta_{s t r}\left\langle(\kappa: \boldsymbol{m} \boldsymbol{m})^{2}\right\rangle
$$

where $\zeta_{s t r}=\frac{\zeta_{r}}{2}$. Then

$$
\left(\tau_{p}^{(V)}\right)_{\alpha \beta}=n \zeta_{s t r} \kappa_{\mu \gamma}\left\langle m_{\mu} m_{\gamma} m_{\alpha} m_{\beta}\right\rangle=\frac{n}{2} \zeta_{r} \kappa_{\mu \gamma}\left\langle m_{\mu} m_{\gamma} m_{\alpha} m_{\beta}\right\rangle
$$

Finally the polymeric stress is given by

$$
\tau_{p}=3 n k_{B} T \boldsymbol{S}-n\langle(\boldsymbol{m} \times \mathcal{R} U) \otimes \boldsymbol{m}\rangle+\frac{n}{2} \zeta_{r} \kappa:\langle\boldsymbol{m} \otimes \boldsymbol{m} \otimes \boldsymbol{m} \otimes \boldsymbol{m}\rangle .
$$


Similar to the dumbbell model, the non-dimensionalization introduces the nondimensional parameter

$$
D e=\frac{T_{r}}{T_{c}},
$$

where $T_{r}=\zeta_{r} /\left(k_{B} T\right)$ is the mesoscopic relaxation time scale, $T_{c}$ is the macroscopic convective time scale which is the same as that in the dumbbell model. The nondimensionalized form for $\psi$ is:

$$
\partial_{t} \psi=\frac{1}{D e} \mathcal{R} \cdot(\mathcal{R} \psi+\psi \mathcal{R} U)-\mathcal{R} \cdot(\boldsymbol{m} \times \kappa \cdot \boldsymbol{m} \psi) .
$$

The polymeric stress has the form

$$
\tau_{p}=3 \boldsymbol{S}-\langle(\boldsymbol{m} \times \mathcal{R} U) \otimes \boldsymbol{m}\rangle+\frac{D e}{2} \kappa:\langle\boldsymbol{m} \otimes \boldsymbol{m} \otimes \boldsymbol{m} \otimes \boldsymbol{m}\rangle .
$$

The corresponding stochastic version of $(2.53)$ is

$$
\frac{\partial \boldsymbol{m}}{\partial t}+\left(\boldsymbol{u} \cdot \nabla_{\boldsymbol{x}}\right) \boldsymbol{m}=(\boldsymbol{I}-\boldsymbol{m} \otimes \boldsymbol{m}) \circ\left(-\frac{1}{D e} \mathcal{R} U+\boldsymbol{m} \times \kappa \cdot \boldsymbol{m}+\sqrt{\frac{2}{D e}} \dot{\boldsymbol{W}}(t)\right) .
$$

where "०" means the Stratonovich integral because the Brownian motion is on the sphere $\mathbb{S}^{2}[32]$.

\section{Mathematical analysis of the models}

3.1. The structure of stationary solutions of the Doi-Onsager equation.

The basic object in the Doi-Onsager equation (3.4) is the single molecule positionorientation distribution function. Interactions between molecules are modelled by a mean-field potential. Therefore, the Doi-Onsager equation can be regarded as a meanfield kinetic theory. Besides interacting with other rods, the rods are also interacting with the flow and are subject to Brownian forces. If the interaction is sufficiently strong compared with the Brownian forces, or if the rod concentration is sufficiently high, the system prefers to be in nematic phase so that the rods tend to line up with each other. Otherwise the system is in isotropic phase where the orientation of the rods is completely random.

The Doi-Onsager equation is known to exhibit nontrivial nonlinear features, e.g., $[8,33]$ and its study has recently attracted great attention, e.g., [21, 61, 63]. The general Doi-Onsager equation describes the rotation and translation of polymeric molecules convected with the flow. A basic feature of this model is its ability to describe both the isotropic and nematic phases, e.g., [13, 14, 70]. The complex dynamical properties are amplified considerably when the phase transition occurs. Such a remarkable phase transition phenomenon in rigid rod-like polymers has been observed in both experiments and numerical simulations, e.g., [21, 28, 61, 63].

We focus our attention on the Doi-Onsager equation on the sphere with the MaierSaupe potential. We study the structure and phase transitions to stationary solutions of the Doi-Onsager equation. Such a phase transition problem was first described by Onsager in 1949 [70], using a variational approach. He used the free energy (3.2), by restricting $\psi$ to be of the form

$$
\psi(\boldsymbol{m})=\frac{\beta}{4 \pi \sinh \beta} \cosh (\beta \boldsymbol{m} \cdot \boldsymbol{n}),
$$


where $\boldsymbol{n} \in \mathbb{S}^{2}$ is a director parameter and $\beta$ is a parameter to be determined by minimizing the free energy. Here $\beta$ represents the degree of ordering: $\beta=0$ corresponds to the isotropic state where $\psi=$ const, and $\beta=\infty$ the completely ordered state where $\psi$ is a Delta function. Onsager was able to argue that in the limit of high concentration, one has a transition from the isotropic uniform distribution to an ordered prolate distribution $[8,70]$.

In the last few years, the Doi-Onsager model has attracted a great deal of attention in the mathematics community $[7,8,9,10,22,23,60,56,88]$. In particular, concerning the structure of stationary solutions of the Doi-Onsager model, Constantin et al. [8] classified these solutions in the high concentration limit. They also proved that the isotropic state is the only possible solution at sufficiently low concentration. The situation is much better understood in the two-dimensional case when the orientation variable lies on the circle. Constantin et al. [9] established a bound on the number of stationary solutions, and at the same time, gave a sharp estimate on the region of stability for the isotropic solution. Luo et al. [60] gave a detailed study of the structure of the stationary solutions, proving that there are only two possible solutions, one corresponding to isotropic state, the other corresponding to the nematic state. Their proof was further simplified in [10, 22].

For the Doi-Onsager model on the sphere with the Maier-Saupe potential, Liu et al. [56] prove that all stationary solutions are axially symmetric, and gave sharp estimates on the region of stability for the isotropic and nematic solutions. Taken together, these results give a rather complete understanding of the Doi-Onsager model on the sphere with Maier-Saupe potential. The axial symmetry was also proved independently by Fatkullin et al. [23].

We consider the equation (2.53) in the absence of flow and under the assumption $D e=1$ :

$$
\frac{\partial \psi}{\partial t}=\mathcal{R} \cdot(\mathcal{R} \psi+\psi \mathcal{R} U)
$$

We recall that Onsager's argument is based on the free energy

$$
A(\psi)=\int_{\mathbb{S}^{2}}\left[\psi(\boldsymbol{m}) \ln \psi(\boldsymbol{m})+\frac{1}{2} \psi(\boldsymbol{m}) U(\boldsymbol{m})\right] d \boldsymbol{m} .
$$

Its Euler-Lagrange equation,

$$
\ln \psi(\boldsymbol{m})+U(\boldsymbol{m})=\text { const. },
$$

can be obtained by integrating the time-independent Doi-Onsager equation

$$
\mathcal{R} \cdot(\mathcal{R} \psi+\psi \mathcal{R} U)=0 .
$$

The Maier-Saupe potential is defined as in equation $(2.42)$, with the parameter $2 b L^{2}$ later replaced by $\alpha$.

The Doi-Onsager equation restricted on a circle is much simpler and is well understood because of recent efforts $[9,10,22,60,56]$. For the two-dimensional case, the Doi-Onsager equation (3.4) with (2.42) reduces to the following:

$$
\begin{aligned}
& \psi_{\theta \theta}+\left(\psi U_{\theta}\right)_{\theta}=0, \quad \theta \in[0,2 \pi], \\
& U(\theta)=\alpha \int_{0}^{2 \pi} \sin ^{2}\left(\theta-\theta^{\prime}\right) \psi\left(\theta^{\prime}\right) d \theta^{\prime},
\end{aligned}
$$


with the normalization

$$
\int_{0}^{2 \pi} \psi(\theta) d \theta=1
$$

Therefore we obtain a decoupled equation (3.8) for $U$,

$$
U_{\theta \theta}+4 U=2 \alpha
$$

Its general solution is

$$
U=\frac{\alpha}{2}+\eta \cos 2\left(\theta-\theta_{0}\right)
$$

where $\eta$ and $\theta_{0}$ are two arbitrary parameters. Without loss of generality we assume $\eta \geq 0$ because a sign change can be always made by simply shifting $\theta_{0}$ to $\theta_{0}+\pi$. With this explicit formula $U$ at hand, we proceed to solve $\psi$ in terms of $U$. Using (3.9) we have

$$
\psi=C e^{-\eta \cos 2\left(\theta-\theta_{0}\right)},
$$

The use of relation (3.7) gives

$$
C(\eta)=\left[\int_{0}^{2 \pi} e^{-\eta \cos 2 \theta} d \theta\right]^{-1}
$$

Combining this with the nonlocal constraint (3.6) we obtain the relation

$$
\frac{\int_{0}^{2 \pi} \sin ^{2}\left(\theta-\theta^{\prime}\right) e^{-\eta \cos 2\left(\theta^{\prime}-\theta_{0}\right)} d \theta^{\prime}}{\int_{0}^{2 \pi} e^{-\eta \cos 2\left(\theta-\theta_{0}\right)} d \theta}=\frac{1}{2}+\frac{\eta}{\alpha} \cos 2\left(\theta-\theta_{0}\right),
$$

which can be further simplified as

$$
\frac{\int_{0}^{2 \pi} \cos 2 \theta e^{-\eta \cos 2 \theta} d \theta}{\int_{0}^{2 \pi} e^{-\eta \cos 2 \theta} d \theta}+\frac{2 \eta}{\alpha}=0 .
$$

It is now clear that the determination of the number of solutions $\psi$ is equivalent to the determination of the number of $\eta$ in terms $\alpha$ since $C$ is uniquely determined by $\eta$ from (3.11). Therefore we just need to determine the number of zeros of $B(\eta, \alpha)$, which is defined by

$$
B(\eta, \alpha)=\frac{\int_{0}^{2 \pi} \cos 2 \theta e^{-\eta \cos 2 \theta} d \theta}{\int_{0}^{2 \pi} e^{-\eta \cos 2 \theta} d \theta}+\frac{2 \eta}{\alpha} .
$$

It is easy to see that $\eta=0$ is always a zero of $B$ for any $\alpha$.

We now state the result on critical intensities of phase transitions and all explicit stationary solutions of the Doi-Onsager equation on the circle.

THEOREM 3.1. The number of stationary solutions to (3.5)-(3.7) depends on whether the intensity crosses the critical value $\alpha=4$. More precisely,

(i) If $\alpha \leq 4$, then the only stationary solution is the constant $f=1 / 2 \pi$. 
(ii) If $\alpha>4$, then besides the constant solution $f=1 / 2 \pi$, all other equilibrium solutions are given by

$$
\psi(\theta)=\frac{e^{-\eta^{*} \cos 2\left(\theta-\theta_{0}\right)}}{\int_{0}^{2 \pi} e^{-\eta^{*} \cos 2 \theta} d \theta},
$$

where $\theta_{0}$ is arbitrary, $\eta^{*}$ is uniquely determined by

$$
\frac{\int_{0}^{2 \pi} \cos 2 \theta e^{-\eta^{*} \cos 2 \theta} d \theta}{\int_{0}^{2 \pi} e^{-\eta^{*} \cos 2 \theta} d \theta}+\frac{2 \eta^{*}}{\alpha}=0 .
$$

Now we consider the Doi-Onsager equation on the sphere with Maier-Saupe potential:

$$
\begin{aligned}
& \mathcal{R} \cdot \mathcal{R} \psi+\mathcal{R} \cdot(\psi \mathcal{R} U)=0, \quad \boldsymbol{m} \in \mathbb{S}^{2}, \\
& U(\boldsymbol{m})=\alpha \int_{\mathbb{S}^{2}}\left|\boldsymbol{m} \times \boldsymbol{m}^{\prime}\right|^{2} \psi\left(\boldsymbol{m}^{\prime}\right) d \boldsymbol{m}^{\prime}
\end{aligned}
$$

with the normalization

$$
\int_{\mathbb{S}^{2}} \psi(\boldsymbol{m}) d \boldsymbol{m}=1
$$

The solution of (3.16) can be expressed as

$$
\psi=C e^{-U}
$$

where $C=\left[\int_{\mathbb{S}^{2}} e^{-U} d \boldsymbol{m}\right]^{-1}$. In order to identify all solutions of $\psi$, we just need to find all solutions of $U$. To do so, we first obtain a decoupled linear equation for $U$. The mean-field interaction potential $U$ defined in (3.17) satisfies a decoupled equation,

$$
\mathcal{R} \cdot \mathcal{R} U+6 U=4 \alpha .
$$

The potential $U$ also has the following important properties. $U(\boldsymbol{m})$ is a solution of (3.17) if and only if it satisfies

$$
U(\boldsymbol{m})=\alpha \int_{\mathbb{S}^{2}}\left|\boldsymbol{m} \times \boldsymbol{m}^{\prime}\right|^{2} e^{-U\left(\boldsymbol{m}^{\prime}\right)} d \boldsymbol{m}^{\prime}\left[\int_{\mathbb{S}^{2}} e^{-U\left(\boldsymbol{m}^{\prime}\right)} d \boldsymbol{m}^{\prime}\right]^{-1} .
$$

Moreover, if $U(\boldsymbol{m})$ is a solution to $(3.21), U(R \boldsymbol{m})$ is also a solution, where $R$ is an arbitrary rotation matrix in $\mathbb{R}^{3}$.

First, it is easy to verify $U=2 \alpha / 3$ is a particular solution of (3.20). In order to obtain the general solution of (3.20) we only need to solve the linear homogenous equation

$$
\mathcal{R} \cdot \mathcal{R} V+6 V=0
$$

where $V=U-\frac{2 \alpha}{3}$. Using the rotational invariance we can always choose an operator $R$ such that $V(\boldsymbol{m})$ can be simplified as

$$
V(T \boldsymbol{m})=\alpha\left(\frac{1}{3}-\sum_{i=1}^{3} q_{i} m_{i}^{2}\right)
$$


where

$$
q_{i} \triangleq q_{i}(V)=\int_{\mathbb{S}^{2}} m_{i}^{2} e^{-V(\boldsymbol{m})} d \boldsymbol{m}\left[\int_{\mathbb{S}^{2}} e^{-V(\boldsymbol{m})} d \boldsymbol{m}\right]^{-1}
$$

is a functional of $V$, up to a rotation.

Since $\mathcal{R} \cdot \mathcal{R}$ is the Laplace-Beltrami operator on the sphere, its eigenfunctions are the spherical harmonics. The space spanned by the eigenfunctions with the eigenvalue -6 has dimension five. Then we have

$$
V(\boldsymbol{m})=a_{1}\left(m_{1}^{2}-m_{2}^{2}\right)+a_{2} m_{1} m_{2}+a_{3} m_{1} m_{3}+a_{4} m_{2} m_{3}+a_{5}\left(m_{3}^{2}-1 / 3\right)
$$

with $a_{1}, a_{2}, a_{3}, a_{4}, a_{5}$ determined by

$$
V(\boldsymbol{m})=\alpha\left(\frac{1}{3}-\sum_{i=1}^{3} q_{i} m_{i}^{2}\right) \quad \text { for } \quad \forall \boldsymbol{m} \in \mathbb{S}^{2} .
$$

Setting $\boldsymbol{m}=(0,0,1)$ in $(3.25)$ with $(3.26)$ we obtain

$$
\alpha\left(\frac{1}{3}-q_{3}\right)=\frac{2 a_{5}}{3} .
$$

Setting $\boldsymbol{m}=\left(\frac{1}{\sqrt{2}}, \frac{1}{\sqrt{2}}, 0\right),(1,0,0)$ and $(0,1,0)$, we have

$$
\begin{aligned}
\frac{a_{2}}{2}-\frac{a_{5}}{3} & =\alpha\left(\frac{1}{3}-\frac{1}{2}\left(q_{1}+q_{2}\right)\right), \\
a_{1}-\frac{a_{5}}{3} & =\alpha\left(\frac{1}{3}-q_{1}\right), \\
-a_{1}-\frac{a_{5}}{3} & =\alpha\left(\frac{1}{3}-q_{2}\right),
\end{aligned}
$$

respectively. These relations lead to $a_{2}=0$. Similarly we can show that $a_{3}=a_{4}=0$. Thus (3.25) and (3.26) reduce to

$$
a_{1}\left(m_{1}^{2}-m_{2}^{2}\right)+a_{5}\left(m_{3}^{2}-\frac{1}{3}\right)=\alpha\left(\frac{1}{3}-\sum_{i=1}^{3} q_{i} m_{i}^{2}\right) .
$$

From (3.27), (3.29) and (3.30) it follows that

$$
\begin{aligned}
& \alpha\left(q_{2}-q_{1}\right)-2 a_{1}=0 \\
& \alpha\left(1-3 q_{3}\right)-2 a_{5}=0
\end{aligned}
$$

and this leads to

$$
\begin{aligned}
& q_{1}=\frac{1}{3}+\frac{1}{\alpha}\left(\frac{a_{5}}{3}-a_{1}\right) \\
& q_{2}=\frac{1}{3}+\frac{1}{\alpha}\left(\frac{a_{5}}{3}+a_{1}\right) \\
& q_{3}=\frac{1}{3}-\frac{2 a_{5}}{3 \alpha} .
\end{aligned}
$$


Using (3.32) and (3.33), we can obtain an $\alpha$-independent equation, namely

$$
a_{1}\left(1-3 q_{3}\right)-a_{5}\left(q_{2}-q_{1}\right)=0 .
$$

It is equivalent to

$$
\begin{aligned}
F\left(a_{1}, a_{5}\right)= & {\left[\int_{\mathbb{S}^{2}}\left[a_{1}\left(1-3 m_{3}^{2}\right)-a_{5}\left(m_{2}^{2}-m_{1}^{2}\right)\right] e^{-a_{1}\left(m_{1}^{2}-m_{2}^{2}\right)-a_{5}\left(m_{3}^{2}-1 / 3\right)} d \boldsymbol{m}\right] } \\
= & 2 \int_{0}^{1} \int_{0}^{2 \pi}\left[a_{1}\left(1-3 z^{2}\right)-a_{5}\left(z^{2}-1\right) \cos 2 \varphi\right] . \\
& \cdot e^{a_{1}\left(z^{2}-1\right) \cos 2 \varphi+a_{5}\left(1 / 3-z^{2}\right)} d \varphi d z \\
= & 0 .
\end{aligned}
$$

It is straightforward to see that

$$
F\left(0, a_{5}\right)=0, \quad F\left(a_{5}, a_{5}\right)=0, \quad F\left(-a_{5}, a_{5}\right)=0 \quad \forall \quad a_{5} \in \mathbb{R} .
$$

The rest of the proof $[56,23]$ boils down to establishing the elementary fact that there are no other zeros of the function $F\left(a_{1}, a_{5}\right)$ besides $a_{1}=0, \pm a_{5}$, whose contour lines are depicted in Figure 7.

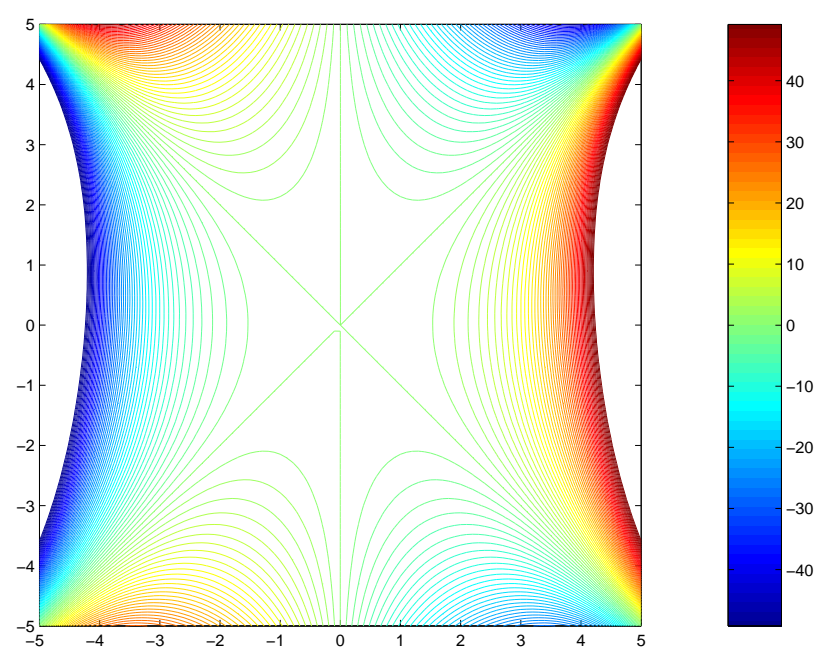

FIG. 3.1. The contour lines of function $F\left(a_{1}, a_{5}\right)$.

Based on this observation, the first result is about the axial symmetry and explicit representations of the potential $U$.

THEOREM 3.2. Consider the Doi-Onsager equation (3.16) with the normalization (3.18). Let $U$ be the potential defined by (3.17). Then such a potential is necessarily invariant with respect to rotations around a director $\boldsymbol{n} \in \mathbb{S}^{2}$, i.e., it is axially symmetric. Moreover, this potential must have the form

$$
U=\frac{2 \alpha}{3}-\eta\left(|\boldsymbol{m} \times \boldsymbol{n}|^{2}-\frac{2}{3}\right),
$$


where $\eta \in \mathbb{R}$ is a parameter.

As the axial symmetry of stationary solutions of the Doi-Onsager equation is obtained, the solutions of (3.16) can be then expressed by

$$
\psi(\boldsymbol{m})=k e^{-\eta(\boldsymbol{m} \cdot \boldsymbol{n})^{2}},
$$

where $\eta, k$ depend on $\alpha$ through the relations:

$$
\begin{aligned}
& \frac{3 e^{-\eta}}{\int_{0}^{1} e^{-\eta z^{2}} d z}-\left(3-2 \eta+\frac{4 \eta^{2}}{\alpha}\right)=0, \\
& k=\left[4 \pi \int_{0}^{1} e^{-\eta z^{2}} d z\right]^{-1} .
\end{aligned}
$$

In order to determine the number of solutions of (3.16), it suffices to determine the number of zeros of $B(\eta, \alpha)$ in terms of $\alpha$, where

$$
B(\eta, \alpha) \triangleq \frac{3 e^{-\eta}}{\int_{0}^{1} e^{-\eta z^{2}} d z}-\left(3-2 \eta+\frac{4 \eta^{2}}{\alpha}\right) .
$$

It can be also written as

$$
\alpha=\frac{2 \eta \int_{0}^{1} e^{-\eta z^{2}} d z}{\int_{0}^{1}\left(1-3 z^{2}\right) e^{-\eta z^{2}} d z}=\frac{\int_{0}^{1} e^{-\eta z^{2}} d z}{\int_{0}^{1}\left(z^{2}-z^{4}\right) e^{-\eta z^{2}} d z},
$$

from which a relation between $\alpha$ and $-\eta$, besides the isotropic case $\eta=0$, can be visualized in Figure 8 . The critical intensity $\alpha^{*}$ can be expressed as

$$
\alpha^{*}=\min _{\eta} \frac{\int_{0}^{1} e^{-\eta z^{2}} d z}{\int_{0}^{1}\left(z^{2}-z^{4}\right) e^{-\eta z^{2}} d z},
$$

which is about $\alpha^{*} \approx 6.731393$ from our numerical calculation. We now state our second result on critical intensities of phase transitions and all explicit stationary distributions, which is proved by Liu et al. [56].

THEOREM 3.3. The number of stationary solutions of the Doi-Onsager equation on the sphere (3.16) with (3.17), (3.18) hinges on whether the intensity $\alpha$ crosses two critical values: $\alpha^{*} \approx 6.731393$ and 7.5 . All solutions are given explicitly by

$$
\psi=k e^{-\eta(\boldsymbol{m} \cdot \boldsymbol{n})^{2}},
$$

where $\boldsymbol{n} \in \mathbb{S}^{2}$ is a parameter, $\eta=\eta(\alpha)$ and $k=\left[4 \pi \int_{0}^{1} e^{-\eta z^{2}} d z\right]^{-1}$ are determined by $\alpha$ through

$$
\frac{3 e^{-\eta}}{\int_{0}^{1} e^{-\eta z^{2}} d z}-\left(3-2 \eta+\frac{4 \eta^{2}}{\alpha}\right)=0 .
$$

More precisely,

(i). If $0<\alpha<\alpha^{*}$, there exists one solution $\psi_{0}=1 / 4 \pi$. $\eta_{1}<0$.

(ii). If $\alpha=\alpha^{*}$, there exist two distinct solutions $\psi_{0}=1 / 4 \pi$ and $\psi_{1}=k_{1} e^{-\eta_{1}(\boldsymbol{m} \cdot \boldsymbol{n})^{2}}$, 


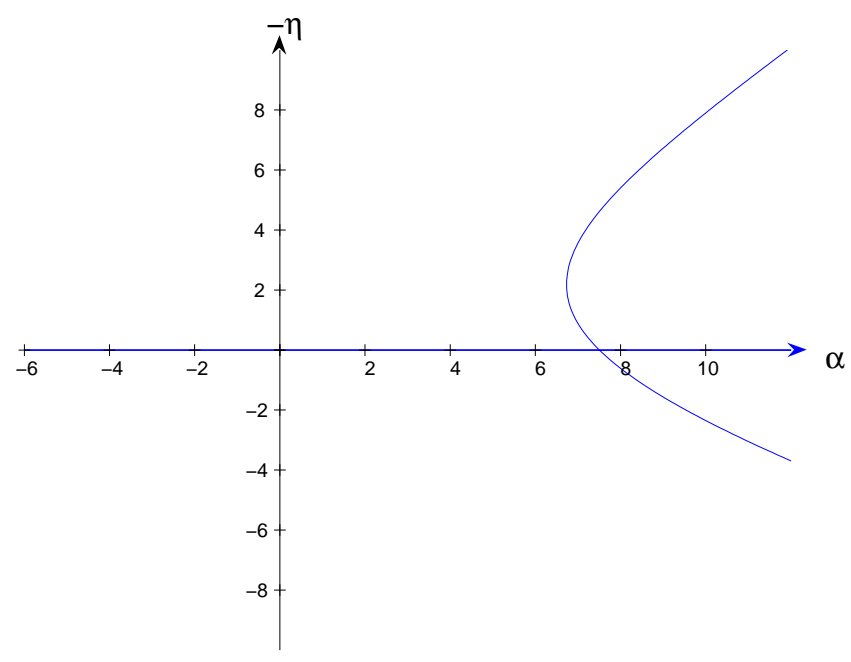

FIG. 3.2. The relation between $\alpha$ and $\eta$.

(iii). If $\alpha^{*}<\alpha<7.5$, there exist three distinct solutions $\psi_{0}=1 / 4 \pi$ and $\psi_{i}=$ $k_{i} e^{-\eta_{i}(\boldsymbol{m} \cdot \boldsymbol{n})^{2}}, \eta_{i}<0(i=1,2)$.

(iv). If $\alpha=7.5$, there exist two distinct solutions $\psi_{0}=1 / 4 \pi$ and $\psi_{1}=$ $k_{1} e^{-\eta_{1}(\boldsymbol{m} \cdot \boldsymbol{n})^{2}}, \eta_{1}<0$.

(v). If $\alpha>7.5$, there exist three distinct solutions $\psi_{0}=1 / 4 \pi$ and $\psi_{i}=k_{i} e^{-\eta_{i}(\boldsymbol{m} \cdot \boldsymbol{n})^{2}}$ $(i=1,2), \eta_{1}<0, \eta_{2}>0$.

In physical terms, an isotropic phase corresponds to the case where the distribution function is $\psi=1 / 4 \pi$ and a nematic phase corresponds to the case when $\psi$ is concentrated at some particular director, which includes the prolate and oblate states. For example, $\psi_{0}=\frac{1}{4 \pi}$ is an isotropic distribution; while the distribution function $\psi_{1}=k_{1} e^{-\eta_{1}(\boldsymbol{m} \cdot \boldsymbol{n})^{2}}\left(\eta_{1}<0\right)$ is concentrated in the direction $\pm \boldsymbol{n}$ (called prolate state) and $\psi_{2}=k_{2} e^{-\eta_{2}(\boldsymbol{m} \cdot \boldsymbol{n})^{2}}\left(\eta_{2}>0\right)$ is concentrated on the equator perpendicular to $\boldsymbol{n}$ (called oblate state).

\subsection{Deterministic approach for the coupled micro-macro model.}

The well-posedness analysis for the macroscopic models of the Oldroyd type maybe originated from Guillope et al. [29]. A local existence and uniqueness for general data and global result for small data is obtained by Schauder's fixed point theorem. A similar result, but based on the equation of the deformation tensor $F$ is obtained in [52]. Lions et al. [54] show the existence of global-in-time weak solutions to the Oldroyd model in a simplified case, which assumes the drag term is corotational, while it is not known if an similar global result also holds in the absence of the corotational assumption.

For the analysis of the coupled micro-macro model, the pioneer Renardy [75] gives a local existence and uniqueness result to a class of general dumbbell models. He considered a slightly different form for incompressible hyper-elastic material which is a hyperbolic system. The basic framework is nice, but some details are missed. It is 
supplemented by Li et al. in [48]. This result does not contain the practically relevant FENE model. Upon smoothing of the convective velocity field in the Fokker-Planck equation, and in some circumstances of the extra-stress tensor, Barrett et al. [2] established the existence of global-in-time weak solutions to the regularised dumbbell model for a general class of spring force potentials including even the FENE model with Dirichlet boundary condition. Liu et al. [53] has recently proved the existence of global classical solutions when the initial data is near the equilibrium configurations for the flow system coupled with the Fokker-Planck equation with Hookean potential. Zhang et al. [85] established well-posedness results of the coupled macro-micro model with the FENE potential under restrictive assumptions on the maximum extension rate, the boundary condition of the FENE-type Fokker-Planck equation is proved to be unnecessary by the singularity on the boundary. Liu et al. [55] show that any boundary condition will become redundant once $H b>2$, where $\sqrt{b}$ is the maximum dumbbell extension. Moreover, the trace of the pdf on the sphere $|\boldsymbol{Q}|=\sqrt{b}$ is necessarily zero when $H b>2$ for any solutions. Du et al. [11] proved the finite time existence of the solution to the FENE-type Fokker-Planck equation with natural boundary condition for general velocity gradients and global existence of the solution in the case where the velocity gradients are close to being purely symmetric or anti-symmetric. We will present the main ideas of these proofs briefly in the next section.

\section{Hookean and general nonlinear dumbbell model}

The simplest spring potential is given by the Hookean law $\Psi(\boldsymbol{Q})=-H Q^{2} / 2$, where $Q=|\boldsymbol{Q}|$ and $H$ is the elastic constant. In this case, one can obtain a closure equation for $\tau_{p}$, which is exactly the Oldroyd-B model. In general, the spring force law must be a nonlinear vector function. Assume the nonlinear force

$$
\boldsymbol{F}(\boldsymbol{Q})=\gamma\left(Q^{2}\right) \boldsymbol{Q}
$$

where $\gamma$ satisfies the following condition:

Assumption: The function $\gamma$ is $C^{\infty}$-smooth from $[0, \infty)$ to $(0, \infty)$, and there exist numbers $\sigma \geq 0$ and $k>0$ such that $\lim _{|\boldsymbol{Q}| \rightarrow \infty} \gamma\left(Q^{2}\right) /|\boldsymbol{Q}|^{\sigma}=k$. Moreover,

$$
\lim \sup _{|\boldsymbol{Q}| \rightarrow \infty}\left|\gamma^{\prime}\left(Q^{2}\right)\right| /|\boldsymbol{Q}|^{\sigma-2} \leq C
$$

and higher derivatives of $\gamma$ have at most polynomial growth as $|\boldsymbol{Q}| \rightarrow \infty$.

This assumption on the spring forces admits a fairly large class of models, including the Hookean dumbbell model, but it excludes the FENE-type models.

The basic idea in $[76,48]$ is to apply an iteration procedure to linearize the nonlinear system, i.e.

$$
\begin{gathered}
\boldsymbol{u}_{t}^{m+1}+\left(\boldsymbol{u}^{m} \cdot \nabla_{\boldsymbol{x}}\right) \boldsymbol{u}^{m+1}+\nabla_{\boldsymbol{x}} p^{m+1}=\Delta_{\boldsymbol{x}} \boldsymbol{u}^{m+1}+\nabla_{\boldsymbol{x}} \cdot \tau^{m} \\
\nabla_{\boldsymbol{x}} \cdot \boldsymbol{u}^{m+1}=0
\end{gathered}
$$

with suitable initial condition and boundary condition, where

$$
\tau^{m}=\int_{\mathbb{R}^{3}} \boldsymbol{F}(\boldsymbol{Q}) \otimes \boldsymbol{Q} \psi^{m} d \boldsymbol{Q}
$$


Meanwhile, for given $\mathbf{u}^{m}$, we determine $\psi^{m+1}$ from

$$
\psi_{t}^{m+1}+\left(\boldsymbol{u}^{m} \cdot \nabla_{\boldsymbol{x}}\right) \psi^{m+1}=-\nabla_{\boldsymbol{Q}} \cdot\left[\left(\kappa^{m} \cdot \boldsymbol{Q}-\boldsymbol{F}(\boldsymbol{Q})\right) \psi^{m+1}\right]+\Delta_{\boldsymbol{Q}} \psi^{m+1} .
$$

The a priori estimate is accomplished with the assumption of spring forces.

The estimate of $\psi$ is composed of two steps:

Step 1: Estimate of $\psi$ w.r.t. Lagrangian variable $\alpha$;

Step 2: Estimate of $\psi$ w.r.t. Eulerian variable $\boldsymbol{x}$.

After linearization, the estimate of $\boldsymbol{u}$ is standard as NSE from the estimate of $\tau$. Note that

$$
\tau=\int_{\mathbb{R}^{3}} \boldsymbol{F}(\boldsymbol{Q}) \otimes \boldsymbol{Q} \psi d \boldsymbol{Q}, \quad \psi \geq 0
$$

The most natural choice of the function space for $\psi$ is a weighted $L^{1}$-space. Then $\psi$ is taken in $\chi_{k}=\cap_{n=0}^{\infty} \chi_{n, k}$, where

$$
\chi_{n, k}=\left\{\psi: \mathbb{R}^{3} \rightarrow \mathbb{R}\left|\int_{\mathbb{R}^{3}}\left(1+|Q|^{n}\right) \sum_{m=0}^{k}\right| \nabla_{\boldsymbol{Q}}^{m} \psi \mid d \boldsymbol{Q}<+\infty\right\}
$$

To derive the estimate of $\psi$ w.r.t. Lagrangian variables, we introduce the flow map

$$
\frac{\partial}{\partial t} \boldsymbol{x}(\boldsymbol{\alpha}, t)=\boldsymbol{u}(\boldsymbol{x}(\boldsymbol{\alpha}, t), t), \quad \boldsymbol{x}(\boldsymbol{\alpha}, 0)=\boldsymbol{\alpha}
$$

and define $\phi(\boldsymbol{\alpha}, \boldsymbol{Q}, t)=\psi(\boldsymbol{x}(\boldsymbol{\alpha}, t), \boldsymbol{Q}, t)$. Thus

$$
\frac{\partial}{\partial t} \phi(\boldsymbol{\alpha}, \boldsymbol{Q}, t)=-\nabla_{\boldsymbol{Q}} \cdot[(\kappa \cdot \boldsymbol{Q}-\boldsymbol{F}(\boldsymbol{Q})) \phi]+\Delta_{\boldsymbol{Q}} \phi
$$

Because the coefficients $\boldsymbol{Q}, \boldsymbol{F}(\boldsymbol{Q})$ are not bounded, standard existence results for parabolic equations cannot be used directly. To overcome this issue, a cut-off function $\chi_{N}(\boldsymbol{Q})$ is applied, for the a priori estimate, it is almost the same without $\chi_{N}(\boldsymbol{Q})$.

Step 1.1: Estimate of $\phi$.

Multiplying both sides of the equation for $\phi$ by $|\boldsymbol{Q}|^{2 n}$ and integrating by parts one obtains

$$
\begin{aligned}
& \frac{\partial}{\partial t} \int_{R^{3}}|\boldsymbol{Q}|^{2 n} \phi d \boldsymbol{Q}=\left(4 n^{2}+2 n\right) \int_{\mathbb{R}^{3}}|\boldsymbol{Q}|^{2 n-2} \phi d \boldsymbol{Q} \\
& \quad+2 n \int_{\mathbb{R}^{3}}|\boldsymbol{Q}|^{2 n-2}\{\boldsymbol{Q} \cdot \kappa \cdot \boldsymbol{Q}-\boldsymbol{Q} \cdot \boldsymbol{F}(\boldsymbol{Q})\} \phi d \boldsymbol{Q}
\end{aligned}
$$

Hence,

$$
\frac{\partial}{\partial t} \int_{\mathbb{R}^{3}}|\boldsymbol{Q}|^{2 n} \phi d \boldsymbol{Q} \leq\left(4 n^{2}+2 n\right) \int_{\mathbb{R}^{3}}|\boldsymbol{Q}|^{2 n-2} \phi d \boldsymbol{Q}+2 n|\kappa| \int_{\mathbb{R}^{3}}|\boldsymbol{Q}|^{2 n} \phi d \boldsymbol{Q} .
$$

Application of Gronwall's inequality yields $\psi \in L^{\infty}\left([0, T], L^{\infty}\left(\Omega, \chi_{0}\right)\right)$.

Step 1.2: Estimate of $\nabla_{\boldsymbol{Q}}^{m} \phi$. 
Notice that $\nabla_{Q}^{m} \phi$ may change sign. Thus, to obtain the $\chi_{n, k}$ bounds, one should consider the positive part and negative part separately. Define $\phi_{+}^{(i)}=\partial_{Q_{i}} \phi \vee 0, \phi_{-}^{(i)}=$ $-\left(\partial_{Q_{i}} \phi \wedge 0\right)$. Then $\phi^{(i)}=\phi_{+}^{(i)}-\phi_{-}^{(i)}$ and

$$
\begin{gathered}
\partial_{t} \phi_{+}^{(i)}=-\nabla_{\mathbf{Q}} \cdot\left[(\kappa \cdot \boldsymbol{Q}-\boldsymbol{F}(\boldsymbol{Q})) \phi_{+}^{(i)}\right]+\Delta_{\boldsymbol{Q}} \phi_{+}^{(i)}+\Theta_{i}^{+} \\
\partial_{t} \phi_{-}^{(i)}=-\nabla_{\mathbf{Q}} \cdot\left[(\kappa \cdot \boldsymbol{Q}-\boldsymbol{F}(\boldsymbol{Q})) \phi_{-}^{(i)}\right]+\Delta_{\boldsymbol{Q}} \phi_{-}^{(i)}+\Theta_{i}^{-},
\end{gathered}
$$

where

$$
\Theta_{i}=-\left(\kappa_{j i}-\partial_{Q_{i}} F_{j}\right) \phi^{(j)}-\nabla_{\boldsymbol{Q}} \cdot\left(\partial_{Q_{i}}(\kappa \boldsymbol{Q}-\boldsymbol{F}(\boldsymbol{Q}))\right) \phi=\Theta_{i}^{+}-\Theta_{i}^{-} .
$$

Here, $\Theta_{i}^{+}$and $\Theta_{i}^{-}$are the positive and negative parts of $\Theta_{i}$ respectively. We have the weighted $L^{1}$ estimate for $\phi_{+}^{(i)}$,

$$
\begin{array}{r}
\frac{\partial}{\partial t} \int_{\mathbb{R}^{3}}|\boldsymbol{Q}|^{2 n} \phi_{+}^{(i)} d \boldsymbol{Q}=\left(4 n^{2}+2 n\right) \int_{\mathbb{R}^{3}}|\boldsymbol{Q}|^{2 n-2} \phi_{+}^{(i)} d \boldsymbol{Q} \\
+2 n \int_{\mathbb{R}^{3}}|\boldsymbol{Q}|^{2 n-2}\{\boldsymbol{Q} \cdot \kappa \cdot \boldsymbol{Q}-\boldsymbol{Q} \cdot \boldsymbol{F}(\boldsymbol{Q})\} \phi_{+}^{(i)} d \boldsymbol{Q}+\int_{\mathbb{R}^{3}}|\boldsymbol{Q}|^{2 n} \Theta_{i}^{+} d \boldsymbol{Q},
\end{array}
$$

where

$$
\begin{aligned}
\int_{\mathbb{R}^{3}}|\boldsymbol{Q}|^{2 n} \Theta_{i}^{+} d \boldsymbol{Q} & \leq \int_{\mathbb{R}^{3}}|\boldsymbol{Q}|^{2 n} \phi\left|\nabla_{\boldsymbol{Q}} \cdot \partial_{Q_{i}}(\kappa \cdot \boldsymbol{Q}-\boldsymbol{F}(\boldsymbol{Q}))\right| d \boldsymbol{Q} \\
& +\int_{\mathbb{R}^{3}}|\boldsymbol{Q}|^{2 n}\left(\phi_{+}^{(j)}+\phi_{-}^{(j)}\right)\left(\left|\kappa_{j i}\right|+\left|\partial_{Q_{i}} F_{j}(\boldsymbol{Q})\right|\right) d \boldsymbol{Q} .
\end{aligned}
$$

Assuming $\|\kappa\|_{L^{\infty}([0, T] \times \Omega)} \leq M$ (which will be justified by estimate of $\boldsymbol{u}$ ), the first and second term in (3.58) may be controlled by a Gronwall type inequality. The first term in (3.59) may be controlled by the estimates in Step 1.1. The only vague term is $\partial_{Q_{i}}\left(F_{j}(\boldsymbol{Q})\right)$ in the second term. One may calculate

$$
\partial_{Q_{i}}\left(F_{j}(\boldsymbol{Q})\right)=\partial_{Q_{i}}\left(\gamma\left(Q^{2}\right) Q_{j}\right)=\gamma^{\prime}\left(Q^{2}\right) 2 Q_{i} Q_{j}+\gamma\left(Q^{2}\right) \delta_{i j} .
$$

Then the negative contribution from the second term in (3.58) gives

$$
-2 n|\boldsymbol{Q}|^{2 n-2} \boldsymbol{Q} \cdot \boldsymbol{F}(\boldsymbol{Q})=-2 n|\boldsymbol{Q}|^{2 n} \gamma\left(Q^{2}\right) .
$$

From the assumption on the force $\boldsymbol{F}$, one has

$$
\gamma\left(Q^{2}\right) \sim k Q^{\sigma}, \quad \gamma^{\prime}\left(Q^{2}\right) \leq C Q^{\sigma-2},
$$

and so

$$
\gamma^{\prime}\left(Q^{2}\right) Q^{2} \leq C \frac{\gamma\left(Q^{2}\right)}{k}, \quad Q \rightarrow+\infty .
$$

Hence the term (3.61) may control the $\partial_{Q_{i}}\left(F_{j}(\boldsymbol{Q})\right)$ term when $n$ is large enough.

The higher order estimate for $\left|\nabla_{Q}^{m} \phi\right|$ is very similar to the above procedure. The estimate w.r.t. Eulerian variable requires the estimate of the flow map.

Step 2: Estimate of flow map.

Notice that

$$
\frac{\partial}{\partial t}\left(\nabla_{\boldsymbol{\alpha}} \boldsymbol{x}\right)=\nabla_{\boldsymbol{x}} \boldsymbol{u} \cdot \nabla_{\boldsymbol{\alpha}} \boldsymbol{x}
$$


and

$$
\frac{\partial}{\partial t} \nabla_{x} \boldsymbol{\alpha}=-\nabla_{\boldsymbol{x}} \boldsymbol{\alpha} \cdot \frac{\partial}{\partial t}\left(\nabla_{\boldsymbol{\alpha}} \boldsymbol{x}\right) \cdot\left(\nabla_{\boldsymbol{\alpha}} \boldsymbol{x}\right)^{-1}
$$

Then one obtains

$$
\frac{\partial}{\partial t} \nabla_{x} \boldsymbol{\alpha}=-\nabla_{\boldsymbol{x}} \boldsymbol{\alpha} \cdot \nabla_{\boldsymbol{x}} \boldsymbol{u}
$$

So if $\kappa \in L^{\infty}([0, T] \times \Omega)$, then $\nabla \boldsymbol{\alpha} \in L^{\infty}([0, T] \times \Omega)$. A similar analysis establishes higher order estimate $\nabla^{2} \boldsymbol{\alpha}, \nabla^{3} \boldsymbol{\alpha} \in L^{\infty}\left([0, T] ; L^{2}(\Omega)\right)$. Combining these results and the estimate of $\phi$ gives the estimate of $\psi$.

\section{FENE dumbbell model}

The techniques introduced by Renardy [76] and refined by Li et al. [48] on dumbbell models cannot be used directly for the FENE problem. Zhang et al. [85] extended the FENE-typed Fokker-Planck equation to the general nonlinear problem with regularized parameter $\varepsilon$. They got some of the estimates independent of the parameter $\varepsilon$ with respect to weighted $L^{1}$ spaces, and showed the well-posedness of the micro-macro models. The boundary condition of the FENE-type Fokker-Planck equation is proved to be unnecessary by the singularity of the FENE potential. Local-in-time existence and uniqueness of a strong solution to Cauchy problem is proven under restrictive assumptions on the maximum extension rate.

After defining $\boldsymbol{Q}=\sqrt{b} \boldsymbol{Q}^{\prime}$ and changing to Lagrangian coordinates $\boldsymbol{\alpha}$, one transforms the equation (2.26) to a equation of $\psi\left(\boldsymbol{\alpha}, \boldsymbol{Q}^{\prime}, t\right)$ (we still denote $\boldsymbol{Q}^{\prime}$ as $\boldsymbol{Q}$ for notational convenience):

$$
\frac{\partial}{\partial t} \psi(\boldsymbol{\alpha}, \boldsymbol{Q}, t)=-\nabla_{\boldsymbol{Q}} \cdot\left[\left(\kappa \cdot \boldsymbol{Q}-\frac{1}{2 D e} \boldsymbol{F}(\boldsymbol{Q})\right) \psi\right]+\frac{1}{2 b D e} \Delta_{\boldsymbol{Q}} \psi,
$$

where $\boldsymbol{F}(\boldsymbol{Q})=\gamma\left(Q^{2}\right) \boldsymbol{Q}$, and $\gamma\left(Q^{2}\right)=\frac{1}{1-Q^{2}}$ is the FENE force.

Firstly, let $\eta$ be a $C^{\infty}$-function $[0,1] \rightarrow[0,1]$ such that $\eta(s)=1$ in a neighborhood of 0 and $\eta(s)=0$ in a neighborhood of 1 , and let $\eta_{\varepsilon}(\boldsymbol{Q})=\eta\left(\frac{2}{\varepsilon}[|\boldsymbol{Q}|-(1-\varepsilon)]\right)$ for $0<\varepsilon<$ $1 / 2$. Then we define

$$
\gamma_{\varepsilon}\left(|\boldsymbol{Q}|^{2}\right)= \begin{cases}\gamma\left(|\boldsymbol{Q}|^{2}\right), & |\boldsymbol{Q}| \leq 1-\varepsilon \\ \gamma\left((1-\varepsilon / 2)^{2}\right), & |\boldsymbol{Q}| \geq 1-\varepsilon / 2 \\ \eta_{\varepsilon}(\boldsymbol{Q}) f\left(|\boldsymbol{Q}|^{2}\right)+\left(1-\eta_{\varepsilon}(\boldsymbol{Q})\right) \gamma\left(\left(1-\frac{\varepsilon}{2}\right)^{2}\right), & \text { otherwise }\end{cases}
$$

Now we set

$$
\boldsymbol{F}_{\varepsilon}(\boldsymbol{Q})=\gamma_{\varepsilon}\left(|\boldsymbol{Q}|^{2}\right) \boldsymbol{Q},
$$

and the extension of $\psi_{0}(\boldsymbol{x}, \boldsymbol{Q})$ is given by

$$
\psi_{0}(\boldsymbol{x}, \boldsymbol{Q})= \begin{cases}\psi_{0}(\boldsymbol{x}, \boldsymbol{Q}), & \text { if }|\boldsymbol{Q}| \leq 1, \\ 0, & \text { if }|\boldsymbol{Q}|>1\end{cases}
$$

Next we will solve the following Cauchy problem:

$$
\begin{aligned}
& \frac{\partial}{\partial t} \phi^{\varepsilon}(\boldsymbol{\alpha}, \boldsymbol{Q}, t)=-\nabla_{\mathbf{Q}} \cdot\left[\left(\kappa \cdot \mathbf{Q}-\frac{1}{2 D e} \boldsymbol{F}_{\varepsilon}(\mathbf{Q})\right) \phi^{\varepsilon}\right]+\frac{1}{2 b D e} \triangle_{\mathbf{Q}} \phi^{\varepsilon} \\
& \phi^{\varepsilon}(\boldsymbol{\alpha}, \boldsymbol{Q}, 0)=\psi_{0}(\boldsymbol{\alpha}, \boldsymbol{Q}) .
\end{aligned}
$$


Define a family of spaces:

$$
\mathcal{X}_{n, i}^{\varepsilon}=\left\{\phi_{\varepsilon}: \mathbb{R} \rightarrow \mathbb{R} \mid \sum_{k=0}^{i}\left[\int_{\mathbb{R}}\left(\sum_{j=0}^{n+5-k} w_{\varepsilon}^{j}\right)\left|\nabla_{\boldsymbol{Q}}^{k} \phi_{\varepsilon}(\boldsymbol{Q})\right| d \boldsymbol{Q}\right]<\infty, n \in \mathbb{N}\right\}
$$

with the norm

$$
\left\|\phi_{\varepsilon}\right\|_{\mathcal{X}_{n, i}^{\varepsilon}}=\sum_{k=0}^{i}\left[\int_{\mathbb{R}}\left(\sum_{j=0}^{n+5-k} w_{\varepsilon}^{j}\right)\left|\nabla_{\boldsymbol{Q}}^{k} \phi_{\varepsilon}(\boldsymbol{Q})\right| d \boldsymbol{Q}\right]
$$

where

$$
w_{\varepsilon}(\boldsymbol{Q})=\left\{\begin{array}{l}
\gamma\left(|\boldsymbol{Q}|^{2}\right)=\frac{1}{1-|\boldsymbol{Q}|^{2}}, \text { if }|\boldsymbol{Q}| \leq 1-\varepsilon \\
A_{1}+A_{2}[|\boldsymbol{Q}|-(1-\varepsilon)], \text { if }|\boldsymbol{Q}|>1-\varepsilon
\end{array}\right.
$$

In (3.73), $A_{1}=\frac{1}{\varepsilon(2-\varepsilon)}$ and $A_{2}=\frac{2(1-\varepsilon)}{\varepsilon^{2}(2-\varepsilon)^{2}}$. The function $w_{\varepsilon}$ and its gradient are continuous at $|\boldsymbol{Q}|=1-\varepsilon$, and $w_{\varepsilon}$ has linear growth at infinity. By the definition of $w_{\varepsilon}(\boldsymbol{Q})$, we can obtain the following result.

We will present here the a priori estimate formally for the essence of the proof. Multiplying equation (3.66) with $\left(\gamma\left(Q^{2}\right)\right)^{n}$ on both sides and integrating in $B_{1}$ one obtains

$$
\begin{aligned}
\frac{\partial}{\partial t} \int_{B_{1}} \gamma^{n} \psi d \boldsymbol{Q}= & D_{2} \int_{B_{1}}\left(4 n(n+1) Q^{2} \gamma^{n+2}+2 n d \gamma^{n+1}\right) \psi d \boldsymbol{Q} \\
& +\int_{B_{1}}\left(\kappa \cdot \boldsymbol{Q}-D_{1} \gamma \boldsymbol{Q}\right) \cdot \boldsymbol{Q} 2 n \gamma^{n+1} \psi d \boldsymbol{Q},
\end{aligned}
$$

where $d$ is the dimension, $D_{2}=1 /(2 b D e)$ and $D_{1}=1 /(2 D e)=b D_{2}$. Collecting the terms appropriately one has

$$
\begin{aligned}
& \frac{\partial}{\partial t} \int_{B_{1}} \gamma^{n} \psi d \boldsymbol{Q}+2 n D_{1}\left(1-\frac{2(n+1)}{b}\right) \int_{B_{1}} Q^{2} \gamma^{n+2} \psi d \boldsymbol{Q} \\
\leq & \left(2 D_{2} n d+2 n|\kappa|\right) \int_{B_{1}} \gamma^{n+1} \psi d \boldsymbol{Q} .
\end{aligned}
$$

In order to control the terms $\int_{B_{1}} \gamma\left(Q^{2}\right)^{n+1} \psi d \boldsymbol{Q}$, we consider the following inequality for the nonnegative $\phi$ :

$$
\begin{aligned}
\int_{B_{1}}\left(\frac{1}{1-Q^{2}}\right)^{n+1} \psi d \boldsymbol{Q} & =\int_{B_{1-\epsilon}}\left(\frac{1}{1-Q^{2}}\right)^{n+1} \psi d \boldsymbol{Q} \\
& +\int_{B_{1} \backslash B_{1-\epsilon}}\left(\frac{1}{1-Q^{2}}\right)^{n+1} \psi d \boldsymbol{Q} \\
& =P_{1}+P_{2} .
\end{aligned}
$$

If $Q \leq 1-\epsilon$, then $\frac{1}{1-Q^{2}} \leq \frac{1}{1-(1-\epsilon)^{2}}=\frac{1}{\epsilon(2-\epsilon)}$; if $Q \geq 1-\epsilon$, then $1-Q^{2} \leq 1-(1-\epsilon)^{2}=$ $\epsilon(2-\epsilon)$. We have

$$
P_{1} \leq C_{1} \int_{B_{1}}\left(\frac{1}{1-Q^{2}}\right)^{n} \psi d \boldsymbol{Q}
$$


and

$$
P_{2} \leq C_{2} \int_{B_{1}} Q^{2}\left(\frac{1}{1-Q^{2}}\right)^{n+2} \psi d \boldsymbol{Q},
$$

where $C_{1}=\frac{1}{\epsilon(2-\epsilon)}, C_{2}=\frac{\epsilon(2-\epsilon)}{(1-\epsilon)^{2}}$. It is easy to find $C_{2} \rightarrow 0$ as $\epsilon \rightarrow 0$. Thus equation (3.75) becomes

$$
\begin{gathered}
\frac{\partial}{\partial t} \int_{B_{1}} \gamma^{n} \psi d \boldsymbol{Q}+\left[2 n D_{1}\left(1-\frac{2(n+1)}{b}\right)-C_{2}\left(2 D_{2} n d+2 n|\kappa|\right)\right] . \\
\cdot \int_{B_{1}} Q^{2} \gamma^{n+2} \psi d \boldsymbol{Q} \leq C_{1}\left(2 D_{2} n d+2 n|\kappa|\right) \int_{B_{1}} \gamma^{n} \psi d \boldsymbol{Q} .
\end{gathered}
$$

One may assume $b>2(n+1)$, and choose $\epsilon$ sufficiently small so that

$$
2 n D_{1}\left(1-\frac{2(n+1)}{b}\right)-C_{2}\left(2 D_{2} n d+2 n|\kappa|\right) \geq 0 .
$$

Then the estimate of $\int_{B} \gamma^{n} \psi d \boldsymbol{Q}$ is a simple application of Gronwall's inequality. Higher order estimates will be similar.

We can get the same estimates independent of the parameter $\varepsilon$ for the extended nonlinear Fokker-Planck equation (3.70).

The complexity with the FENE potential lies with the singularity of the equation at the boundary. Liu et al. [55] discusses the boundary condition for the FokkerPlanck equation (3.81) alone, with the fluid velocity being steady and homogeneous. The velocity gradient is treated as a constant matrix. The key to their approach is to rewrite the equation into a second order equation having standard nonnegative characteristic form, for which they apply the Fichera function criterion to check when boundary conditions are unnecessary. They further investigate the trace of the PDF on the sphere $|\boldsymbol{Q}|=\sqrt{b}$ where no data is pre-imposed. Their approach is to convert the equation by a delicate transformation in such a way that the resulting equation supports a maximum principle.

The well-posedness of the FENE model with natural boundary condition is established by Du et al. [11] under the influence of a steady flow field using energy estimates in configuration space. They constrain the model by studying the FokkerPlanck equation alone decoupled from flow calculation.

The FENE potential that takes into account a finite-extensibility constraint,

$$
\Psi(\boldsymbol{Q})=-\left(H Q_{0}^{2} / 2\right) \log \left(1-\left(Q / Q_{0}\right)^{2}\right),
$$

there does not exist an exact macroscopic constitutive equation for $\tau_{p}$, and thus the FENE model represents a truly multiscale model. Here $Q_{0}$ is the maximum dumbbell extension. The FENE spring force law reads

$$
\boldsymbol{F}(\boldsymbol{Q})=\nabla_{\boldsymbol{Q}} \Psi=\frac{H \boldsymbol{Q}}{1-\left(Q / Q_{0}\right)^{2}} .
$$

Upon applying a standard scaling, the Fokker-Planck equation reduces to

$$
\frac{\partial \psi}{\partial t}+\nabla_{\boldsymbol{Q}} \cdot(\kappa \boldsymbol{Q} \psi)=\frac{1}{2}\left(\nabla_{\boldsymbol{Q}} \cdot\left(\nabla_{\boldsymbol{Q}} \Psi \psi\right)+\Delta_{\boldsymbol{Q}} \psi\right)
$$


where the FENE potential is

$$
\nabla_{Q} \Psi=\frac{H Q}{1-Q^{2} / b}
$$

The Fokker-Planck equation is defined on the open ball $\Omega=\left\{\boldsymbol{Q} \in \mathcal{R}^{n}: Q^{2}<b\right\}$. The equation (3.81) needs to be conservative and the major difficulty is to assign a meaning to the formal natural boundary condition

$$
\left.\left(\frac{1}{2}\left(\nabla_{\boldsymbol{Q}} \Psi \psi+\nabla_{\boldsymbol{Q}} \psi\right)-\kappa \boldsymbol{Q} \psi\right) \cdot \boldsymbol{n}\right|_{\partial \Omega}=0
$$

The spring force is singular at the boundary of $\partial \Omega$. To circumvent this problem, we introduce the following transformation,

$$
\psi(\boldsymbol{Q}, t)=e^{-\Psi(\boldsymbol{Q}) / 2} g(\boldsymbol{Q}, t)
$$

It is easy to check that $g(\boldsymbol{Q}, t)$ satisfies

$$
\frac{\partial g}{\partial t}+\nabla_{\boldsymbol{Q}} \cdot(\kappa \boldsymbol{Q} g)-\frac{\nabla_{\boldsymbol{Q}} \Psi \cdot \kappa \boldsymbol{Q}}{2} g=\frac{1}{4}\left(\Delta_{\boldsymbol{Q}} \Psi-\frac{1}{2}\left|\nabla_{\boldsymbol{Q}} \Psi\right|^{2}\right) g+\frac{1}{2} \Delta_{\boldsymbol{Q}} g .
$$

Terms involving $\nabla_{\boldsymbol{Q}} \Psi \cdot \nabla_{\boldsymbol{Q}} g$ have been cancelled, and the cancellation makes it easier to define the proper space in which the existence of the solution to (3.83) is proved.

In terms of the new function $g$ and the no flux boundary condition (3.82), the singularity caused by $\nabla_{\boldsymbol{Q}} \Psi$ has been removed as long as $b>4$ since $e^{-\frac{\Psi}{2}}=\left(1-Q^{2} / b\right)^{b / 4}$. This constraint on $b$ is not optimal mathematically; it is known that the solution to the stochastic differential equation associated with (3.81) exists and has trajectorial uniqueness if and only if $b>2$. Through the transformation, the solution of the Fokker-Planck equation (3.81) automatically satisfies the natural boundary condition, given certain regularity properties on $g$ near the domain boundary. In particular, to prevent boundary terms from arising when carrying out integration by parts, they impose an extra homogeneous Dirichlet boundary condition on $g$. This does not affect the quality of the result because they are establishing the existence of the solution under more restrictive conditions.

Let $V=\left\{g: \int_{\Omega}\left|\frac{g}{1-Q^{2} / b}\right|^{2} d \boldsymbol{Q}<\infty\right\}$, which is equipped with the norm

$$
\|g\|_{V}=\left(\int_{\Omega}\left|\frac{g}{1-Q^{2} / b}\right|^{2} d \boldsymbol{Q}\right)^{\frac{1}{2}} .
$$

The function space $H_{0}^{1}(\Omega) \cap V$ has the norm $\|g\|_{H^{1}}+\|g\|_{V}$, and we define the space $X=L^{\infty}\left([0, T] ; L^{2}(\Omega)\right) \cap L^{2}\left([0, T] ; H_{0}^{1}(\Omega) \cap V\right) . g \in X$ is a weak solution to (3.81) if and only if $\forall h \in H_{0}^{1}(\Omega) \cap V$, in the sense of distribution,

$$
\begin{aligned}
\frac{d}{d t}(g, h)= & \int_{\Omega} \frac{1}{4}\left(\Delta_{\boldsymbol{Q}} \Psi-\frac{1}{2}\left|\nabla_{\boldsymbol{Q}} \Psi\right|^{2}\right) g h d \boldsymbol{Q}-\frac{1}{2} \int_{\Omega} \nabla_{\boldsymbol{Q}} g \cdot \nabla_{\boldsymbol{Q}} h d \boldsymbol{Q} \\
& +\int_{\Omega}\left(\frac{1}{2} \nabla_{\boldsymbol{Q}} \Psi g-\nabla_{\boldsymbol{Q}} g\right) \cdot \kappa \boldsymbol{Q} h d \boldsymbol{Q},
\end{aligned}
$$

and $g$ satisfies the initial condition

$$
g(0, \boldsymbol{Q})=g_{0}(\boldsymbol{Q})
$$


A priori energy estimates:

$$
\frac{d}{d t}\|g\|_{L^{2}}^{2}=-\frac{1}{2} \int_{\Omega}\left|\frac{1}{2} \nabla_{\boldsymbol{Q}} \Psi g+\nabla_{\boldsymbol{Q}} g\right|^{2} d \boldsymbol{Q}+\int_{\Omega}\left(\frac{1}{2} \nabla_{\boldsymbol{Q}} \Psi g+\nabla_{\boldsymbol{Q}} g\right) \cdot \kappa \boldsymbol{Q} g d \boldsymbol{Q}
$$

can be established by using the fact that $\int_{\Omega} \nabla_{\boldsymbol{Q}} g \cdot \kappa \boldsymbol{Q} g d \boldsymbol{Q}=0$ because $\kappa$ is traceless and $g$ satisfies the zero boundary conditions. Thus, $g \in L^{\infty}\left([0, T] ; L^{2}(\Omega)\right)$ and

$$
\int_{0}^{T} \int_{\Omega}\left|\frac{1}{2} \nabla_{\boldsymbol{Q}} \Psi g+\nabla_{\boldsymbol{Q}} g\right|^{2} d \boldsymbol{Q} d t
$$

is bounded. Using a detailed analysis near the boundary of the domain, we can show that both

$$
\int_{0}^{T} \int_{\Omega}\left|\frac{g}{1-Q^{2} / b}\right|^{2} d \boldsymbol{Q} d t
$$

and

$$
\int_{0}^{T} \int_{\Omega}\left|\nabla_{\boldsymbol{Q}} g\right|^{2} d \boldsymbol{Q} d t
$$

are bounded, as is $g \in L^{2}\left([0, T], H_{0}^{1}(\Omega) \cap V\right)$.

Using the Galerkin method and the a priori energy estimates, it is easy to get the well-posedness of the Fokker-Planck equation (3.81).

Barrett et al. [2] established the existence of global-in-time weak solutions to the coupled microscopic-macroscopic model (2.20)-(2.22) and (2.26). Their hypotheses on the potential $\Psi$ admit a fairly large class of models, including the Hookean dumbbell model as well as general FENE-type models. They do not assume that the flow is corotational in the FENE case; however, in order to complete their existence proofs, the velocity field appearing in the drift-term of the Fokker-Planck equation (2.26) had to be suitably mollified in the case of corotational microscopic-macroscopic models, and in the case of general, non-corotational models, the extra-stress tensor $\tau_{p}$ on the right hand side of (2.20) had to be mollified also.

The velocity $\boldsymbol{v}=S_{\alpha} \boldsymbol{u}$ is a smoothing of $\boldsymbol{u}$ defined as the solution of the following Helmholtz-Stokes problem,

$$
\begin{aligned}
\boldsymbol{v}-\alpha \Delta_{\boldsymbol{x}} \boldsymbol{v}+\nabla_{\boldsymbol{x}} \pi & =\boldsymbol{u} \\
\nabla_{\boldsymbol{x}} \cdot \boldsymbol{v} & =0 \\
\left.\boldsymbol{v}\right|_{\partial \Omega} & =0 .
\end{aligned}
$$

where $\pi$ is a pressure-like auxiliary variable. $\pi$ does not have physical meaning; it is best thought of as a Lagrange multiplier whose role is to enforce incompressibility.

The probability density function $\psi$ satisfies the Fokker-Planck equation together with suitable boundary and initial condition

$$
\frac{\partial \psi}{\partial t}+\boldsymbol{u} \cdot \nabla_{\boldsymbol{x}} \psi+\nabla_{\boldsymbol{Q}} \cdot(\sigma(\boldsymbol{u}) \boldsymbol{Q} \psi)=\frac{1}{2 D e} \nabla_{\boldsymbol{Q}} \cdot\left(\nabla_{\boldsymbol{Q}} \psi+\boldsymbol{F}(\boldsymbol{Q}) \psi\right)
$$

The $\sigma$ is related to $\nabla \boldsymbol{u}$; possible choices are
(i) $\sigma(\boldsymbol{u})=\kappa=\left(\nabla_{\boldsymbol{x}} \boldsymbol{u}\right)^{T}$,
(ii) $\sigma(\boldsymbol{u})=\omega(\boldsymbol{u})$,
(iii) $\sigma(\boldsymbol{u})=\left(\nabla_{\boldsymbol{x}} \boldsymbol{v}\right)^{T}$ 
where

$$
\nabla_{\boldsymbol{x}} \boldsymbol{u}=D(\boldsymbol{u})+\omega(\boldsymbol{u}), \quad D(\boldsymbol{u})=\frac{1}{2}\left(\nabla_{\boldsymbol{x}} \boldsymbol{u}+\left(\nabla_{\boldsymbol{x}} \boldsymbol{u}\right)^{T}\right), \quad \omega(\boldsymbol{u})=\frac{1}{2}\left[\nabla_{\boldsymbol{x}} \boldsymbol{u}-\left(\nabla_{\boldsymbol{x}} \boldsymbol{u}\right)^{T}\right]
$$

On introducing the normalised Maxwellian

$$
M(\boldsymbol{Q})=\frac{e^{-\Psi(\boldsymbol{Q})}}{\int_{\Omega} e^{-\Psi} d \boldsymbol{Q}}
$$

the Fokker-Planck equation can be written as

$$
\frac{\partial \psi}{\partial t}+\boldsymbol{u} \cdot \nabla_{\boldsymbol{x}} \psi+\nabla_{\boldsymbol{Q}} \cdot(\sigma(\boldsymbol{u}) \boldsymbol{Q} \psi)=\frac{1}{2 D e} \nabla_{\boldsymbol{Q}} \cdot\left(M \nabla_{\boldsymbol{Q}}\left(\frac{\psi}{M}\right)\right)
$$

The usual functional spaces for viscous incompressible flow are defined as follows:

$$
\begin{aligned}
H & =\left\{\boldsymbol{v} \in L^{2}(D): \nabla_{\boldsymbol{x}} \cdot \boldsymbol{v}=0\right\}, \\
V & =\left\{\boldsymbol{v} \in H_{0}^{1}(D): \nabla_{\boldsymbol{x}} \cdot \boldsymbol{v}=0\right\}, \\
L_{0}^{2}(D) & =\left\{v \in L^{2}(D): \int_{D} v d \boldsymbol{x}=0\right\} .
\end{aligned}
$$

In addition, we introduce the following function space for $\psi$ :

$$
K=\left\{\phi \in L_{l o c}^{1}(D \times \Omega): \int_{D \times \Omega}\left[\frac{|\phi|^{2}}{M}+M\left|\nabla_{\boldsymbol{Q}}\left(\frac{\phi}{M}\right)\right|^{2}\right] d \boldsymbol{Q} d \boldsymbol{x}<\infty\right\} .
$$

Barrett et al. [2] proved the existence of a global-in-time solution to each of the following weak formulations of these "smoothed" corotational and non-corotational models for any fixed regularization parameter $\alpha>0$ under the following assumptions on data:

$$
\partial D \in C^{2}, \quad \boldsymbol{u}_{0} \in H
$$

and

$$
M^{-\frac{1}{2}} \psi_{0} \in L^{2}(D \times \Omega)
$$

Corotational models: Given $T>0$, and $D, \quad \boldsymbol{u}_{0}$ and $\psi_{0}$, find $\boldsymbol{u} \in$ $L^{\infty}\left([0, T], L^{2}(D)\right) \cap L^{2}([0, T], V) \cap W^{1, \frac{4}{d}}\left([0, T], V^{\prime}\right)$ and $\psi \in L^{2}([0, T], K)$, with $S_{\alpha} \boldsymbol{u} \in$ $L^{2}\left([0, T], W^{1, \infty}(D)\right), M^{-\frac{1}{2}} \psi \in L^{\infty}\left([0, T], L^{2}(D \times \Omega)\right)$ and $\tau_{p} \in L^{\infty}\left([0, T], L^{2}(D)\right)$, such that $\boldsymbol{u}(\cdot, 0)=\boldsymbol{u}_{0}(\cdot)$, and

$$
\begin{aligned}
& \int_{0}^{T} \int_{D}\left[\frac{\partial \boldsymbol{u}}{\partial t} \cdot \boldsymbol{w}+\left(\boldsymbol{u} \cdot \nabla_{\boldsymbol{x}} \boldsymbol{u}\right) \cdot \boldsymbol{w}+\nabla_{\boldsymbol{x}} \boldsymbol{u}: \nabla_{\boldsymbol{x}} \boldsymbol{w}\right] d \boldsymbol{x} d t \\
= & -\int_{0}^{T} \int_{D} \tau_{p}: \nabla_{\boldsymbol{x}} \boldsymbol{w} d \boldsymbol{x} d t, \quad \forall \boldsymbol{w} \in L^{\frac{4}{4-d}}([0, T], V) ; \\
& -\int_{0}^{T} \int_{D \times \Omega} \frac{\psi}{M}\left[\frac{\partial \phi}{\partial t}+\boldsymbol{u} \cdot \nabla_{\boldsymbol{x}} \phi\right] d \boldsymbol{Q} d \boldsymbol{x} d t-\int_{D \times \Omega} \frac{\psi_{0}(\boldsymbol{x}, \boldsymbol{Q})}{M} \phi(\boldsymbol{x}, \boldsymbol{Q}, 0) d \boldsymbol{Q} d \boldsymbol{x} \\
& +\int_{0}^{T} \int_{D \times \Omega}\left[\frac{M}{2 D e} \nabla_{\boldsymbol{Q}}\left[\frac{\psi}{M}-\omega\left(S_{\alpha} \boldsymbol{u}\right) \boldsymbol{Q} \psi\right] \cdot \nabla_{\boldsymbol{Q}}\left(\frac{\phi}{M}\right) d \boldsymbol{Q} d \boldsymbol{x} d t=0, \quad \forall \phi\right.
\end{aligned}
$$


The only difference between (3.91), (3.92) and the corresponding weak formulation of the original corotational model, with $\sigma(\boldsymbol{u})=\omega(\boldsymbol{u})$, is that $\boldsymbol{u}$ has been replaced by $S_{\alpha} \boldsymbol{u}$ in (3.92).

Non-corotational models: Given $T>0$, and $D, \boldsymbol{u}_{0}$ and $\psi_{0}$, find $\boldsymbol{u} \in$ $L^{\infty}\left([0, T], L^{2}(D)\right) \cap L^{2}([0, T], V) \cap W^{1, \frac{4}{d}}\left([0, T], V^{\prime}\right)$ and $\psi \in L^{2}([0, T], K)$, with $S_{\alpha} \boldsymbol{u} \in$ $L^{2}\left([0, T], W^{1, \infty}(D)\right), M^{-\frac{1}{2}} \psi \in L^{\infty}\left([0, T], L^{2}(D \times \Omega)\right)$ and $\tau_{p} \in L^{\infty}\left([0, T], L^{2}(D)\right)$, such that $\boldsymbol{u}(\cdot, 0)=\boldsymbol{u}_{0}(\cdot)$, and

$$
\begin{aligned}
& \int_{0}^{T} \int_{D}\left[\frac{\partial \boldsymbol{u}}{\partial t} \cdot \boldsymbol{w}+\left(\boldsymbol{u} \cdot \nabla_{\boldsymbol{x}} \boldsymbol{u}\right) \cdot \boldsymbol{w}+\nabla_{\boldsymbol{x}} \boldsymbol{u}: \nabla_{\boldsymbol{x}} \boldsymbol{w}\right] d \boldsymbol{x} d t \\
= & -\int_{0}^{T} \int_{D} \tau_{p}: \nabla_{\boldsymbol{x}}\left(S_{\alpha} \boldsymbol{w}\right) d \boldsymbol{x} d t, \quad \forall \boldsymbol{w} \in L^{\frac{4}{4-d}}([0, T], V) \\
& -\int_{0}^{T} \int_{D \times \Omega} \frac{\psi}{M}\left[\frac{\partial \phi}{\partial t}+\left(S_{\alpha} \boldsymbol{u}\right) \cdot \nabla_{\boldsymbol{x}} \phi\right] d \boldsymbol{Q} d \boldsymbol{x} d t-\int_{D \times \Omega} \frac{\psi_{0}(\boldsymbol{x}, \boldsymbol{Q})}{M} \phi(\boldsymbol{x}, \boldsymbol{Q}, 0) d \boldsymbol{Q} d \boldsymbol{x} \\
& +\int_{0}^{T} \int_{D \times \Omega}\left[\frac{M}{2 D e} \nabla_{\boldsymbol{Q}}\left[\frac{\psi}{M}-\nabla_{\boldsymbol{x}}\left(S_{\alpha} \boldsymbol{u}\right) \boldsymbol{Q} \psi\right] \cdot \nabla_{\boldsymbol{Q}}\left(\frac{\phi}{M}\right) d \boldsymbol{Q} d \boldsymbol{x} d t=0, \forall \phi .\right.
\end{aligned}
$$

The only difference between (3.91), (3.92) and (3.93), (3.94) is that the corotational tensor $\omega\left(S_{\alpha} \boldsymbol{u}\right)$ in (3.92) is replaced by the more physical noncorotational tensor $\nabla\left(S_{\alpha} \boldsymbol{u}\right)$ in (3.94) and we applied smoothing on the right-hand side of (3.93) and the corresponding weak formulation of the original noncorotational model, with $\sigma(\boldsymbol{u})=\nabla \boldsymbol{u}$, differ only to the extent that $\boldsymbol{u}$ has been replaced by $S_{\alpha} \boldsymbol{u}$ in (3.92) and we applied smoothing on the right-hand side of (3.93).

This idea and approach are further proceeded in [3]. In their new refined model, the finite size effect of the dumbbells are kept. As a result, the equation for $\psi$ has an extra diffusion term with respect to $\boldsymbol{x}$, and the strain rate is replaced by the differential of the mollifier of $\boldsymbol{u}$ (see equation (2.30)). In [3], they separate the mollifier parameter $\alpha$ in $\mathcal{A}_{\alpha}^{\boldsymbol{x}}$ with the micro-macro size ratio $\varepsilon$. Interestingly, they proved the global existence of weak solution to this complete model when $\alpha>0$ and $\varepsilon>0$. In particular, they rigorously pass to the limit $\varepsilon \rightarrow 0+$ in both the corotational and the general noncorotational case; in the later case only the physically bounded domain problem is proved. By passing to the limit $\alpha \rightarrow 0+$, they prove the wellposedness only in the corotational case. The justification of the simultaneous model reduction $(\alpha, \varepsilon)=(0,0)$ still remains an open problem.

3.3. Stochastic approach for the coupled micro-macro model. The simplest model to account for non-interacting flexible polymer chains in dilute limit is the dumbbell model. The governing equations are a coupled system of incompressible Navier-Stokes equations and stochastic differential equations (2.20)-(2.25). This new type of system raises many interesting problems to mathematicians. As a first step, E et al. [17] and Jourdain et al. [37] analyzed the Hookean dumbbell model in a simple shear flow. The global-in-time existence and uniqueness for the the Cauchy problem has been proved, and the corresponding numerical analysis is done. Jourdain et al. [38] studied the existence of solutions to the FENE model in the case of a simple shear flow. By using some tools in stochastic analysis, they established the local-in-time existence and uniqueness of the solution to the FENE model. In the high-dimensional case, the local-in-time existence and uniqueness is proved by $\mathrm{E}$ et al. in [18] under the polynomial growth condition of the spring force and its derivatives.

For the stochastic model of liquid crystals in the concentrated case, it is closely related to the weakly interacting particle system in probability theory because of 
its mean field character. As a first step, it is proved that the local well-posedness and numerical convergence under shear flow by Li et al. [48]. It was also found by Jourdain et al. [34] that the discretized FENE-P model is also a weakly interacting particle system. Here "-P" means Peterlin's pre-averaging closure technique to the FENE model, in which the FENE force is replaced by

$$
\boldsymbol{F}=\frac{H Q}{1-\left\langle Q^{2}\right\rangle / b} .
$$

The convergence of the stress $\tau_{p}$ with stochastic discretization is obtained.

The well-posedness analysis for the coupled micro-macro system is the main focus of this section. We will present the key ingredients in these proofs one by one.

\section{Hookean dumbbell model under shear flow}

The analysis of shear flow for Hookean dumbbell polymers was first performed in [37]. Now the equations read:

$$
\begin{aligned}
\partial_{t} u & =u_{y y}+\tau_{y}+f_{\text {ext }}, \\
\tau & =\left\langle P_{t} Q_{t}\right\rangle, \\
d P_{t} & =\left(u_{y} Q_{t}-\frac{P_{t}}{2}\right) d t+d V_{t}, \\
d Q_{t} & =-\frac{Q_{t}}{2} d t+d W_{t}
\end{aligned}
$$

with suitable initial and boundary conditions such as

$$
u(0, t)=u(1, t)=0,\left.\quad u\right|_{t=0}=u_{0}(y),\left.\quad P_{t}\right|_{t=0}=P_{0},\left.\quad Q_{t}\right|_{t=0}=Q_{0} .
$$

Here we adopt the conventional subscript $t$ to denote stochastic variables. And $\boldsymbol{u}=$ $(u, 0), \boldsymbol{Q}=\left(P_{t}, Q_{t}\right), \tau=\left(\tau_{p}\right)_{12}$ as in equations (2.20), (2.22) and (2.25).

It is a standard procedure to prove the existence by a Galerkin method. Let us assume $f_{\text {ext }}=0$ and show the formal a priori energy estimate for the essence of the proof. Notice that here the proof is relatively trivial since $P_{t}, Q_{t}$ can be obtained analytically in closed form because of the linearity of SDE.

The first energy estimate may be obtained by multiplying both sides of (3.95) by $u(y, t)$ and taking the integral in space and time:

$$
\frac{1}{2} \frac{d}{d t}\|u\|_{L_{y}^{2}}^{2}+\left\|u_{y}\right\|_{L_{y}^{2}}^{2}=-\int_{0}^{1} \tau u_{y} d y .
$$

By Ito's formula we have

$$
d P_{t}^{2}=2 P_{t} d P_{t}+d t=\left(2 u_{y} P_{t} Q_{t}-P_{t}^{2}\right) d t+2 P_{t} d V_{t}+d t .
$$

Taking expectation and integrating over $[0,1]$ shows that

$$
\frac{1}{2} \frac{d}{d t}\|u\|_{L_{y}^{2}}^{2}+\left\|u_{y}\right\|_{L_{y}^{2}}^{2}+\frac{1}{2} \frac{d}{d t}\left\|P_{t}\right\|_{L_{y}^{2} L_{\omega}^{2}}^{2}+\frac{1}{2}\left\|P_{t}\right\|_{L_{y}^{2} L_{\omega}^{2}}^{2}=\frac{t}{2} .
$$

Thus,

$$
u \in L_{t}^{\infty}\left(L_{y}^{2}\right) \cap L_{t}^{2}\left(H_{y}^{1}\right) .
$$


Differentiating (3.95) w.r.t. space and using a similar energy estimate shows

$$
\frac{d}{d t}\left\|u_{y}\right\|_{L_{y}^{2}}^{2}+\left\|u_{y y}\right\|_{L_{y}^{2}}^{2} \leq\left\|\tau_{y}\right\|_{L_{y}^{2}}^{2} .
$$

Again Ito's formula shows that

$$
d\left(P_{t} Q_{t}\right)=P_{t} d Q_{t}+Q_{t} d P_{t}=\left(u_{y} Q_{t}^{2}-P_{t} Q_{t}\right) d t+Q_{t} d V_{t}+P_{t} d W_{t}
$$

Taking expectation and a standard application of Grownwall's Lemma yields

$$
\left\|\tau_{y}\right\|_{L_{y}^{2}}^{2}=\left\|\left\langle P_{t} Q_{t}\right\rangle_{y}\right\|_{L_{y}^{2}}^{2} \leq \int_{0}^{t}\left\|u_{y y}\right\|_{L_{y}^{2}}^{2} d s .
$$

Substituting this inequality into (3.103), and applying Grownwall's Lemma again we obtain the second energy estimate.

\section{FENE model under shear flow}

After the analysis of shear flow for the Hookean dumbbell, a natural extension is the FENE model. The well-posedness analysis of shear flow for FENE was first considered in [38]. A very nice argument to consider the non-explosive behavior of $\boldsymbol{Q}_{t}$ is presented.

The equations read:

$$
\begin{aligned}
\partial_{t} u & =u_{y y}+\tau_{y} \\
\tau & =\left\langle\frac{P_{t}^{u} Q_{t}^{u}}{1-\frac{\left(P_{t}^{u}\right)^{2}+\left(Q_{t}^{u}\right)^{2}}{b}}\right\rangle, \\
d P_{t}^{u} & =\left(-\frac{1}{2} \frac{P_{t}^{u}}{1-\frac{\left(P_{t}^{u}\right)^{2}+\left(Q_{t}^{u}\right)^{2}}{b}}+u_{y} Q_{t}^{u}\right) d t+d V_{t}, \\
d Q_{t}^{u} & =\left(-\frac{1}{2} \frac{Q_{t}^{u}}{1-\frac{\left(P_{t}^{u}\right)^{2}+\left(Q_{t}^{u}\right)^{2}}{b}}\right) d t+d W_{t},
\end{aligned}
$$

with suitable initial and boundary conditions. Here $b=\left|\boldsymbol{Q}_{0}\right|^{2}$.

The key idea in [38] is to apply Girsanov's transformation to eliminate the $u_{y}$ dependence in $P_{t}^{u}$ 's equation.

For the stress term, we have from Girsanov's transformation:

$$
\mathbb{E}\left(\frac{P_{t}^{u} Q_{t}^{u}}{1-\frac{\left(P_{t}^{u}\right)^{2}+\left(Q_{t}^{u}\right)^{2}}{b}}\right)=\mathbb{E}\left(\frac{P_{t} Q_{t}}{1-\frac{\left(P_{t}\right)^{2}+\left(Q_{t}\right)^{2}}{b}} \mathcal{E}\right)
$$

where $P_{t}, Q_{t}$ denotes $P_{t}^{0}, Q_{t}^{0}\left(u_{y}=0\right.$ case $)$ and $\mathcal{E}$ is the exponential martingale,

$$
\mathcal{E}=\exp \left(\int_{0}^{t} u_{y}(s) Q_{s} d V_{s}-\frac{1}{2} \int_{0}^{t}\left(u_{y}(s) Q_{s}\right)^{2} d s\right),
$$

which means if we have a suitable estimate for $P_{t}, Q_{t}$, we will obtain the estimate for $\mathcal{E}$, and thus for the stress.

Their proof is composed of three steps:

Step 1: the well-posedness for the $P_{t}, Q_{t}$ equation;

Step 2: the well-posedness for the $P_{t}^{u}, Q_{t}^{u}$ equation; 
Step 3: the well-posedness for the coupled system.

In step 1 , the key-point is to prove that the solution vector $\left(P_{t}, Q_{t}\right)$ does not touch the boundary of the ball $B=\left\{(p, q), p^{2}+q^{2}<b\right\}$. This is verified by a stopping time argument and Feller's test for explosion[40] under the condition $b>2$.

Define the stopping time

$$
\tau_{n}=\inf \left\{t:\left(P_{t}^{n}\right)^{2}+\left(Q_{t}^{n}\right)^{2} \geq b\left(1-\frac{1}{n}\right)\right\}
$$

and

$$
\begin{aligned}
& d P_{t}^{n}=\left(-\frac{1}{2} \frac{P_{t}^{n}}{\max \left(1-\frac{\left(P_{t}^{n}\right)^{2}+\left(Q_{t}^{n}\right)^{2}}{b}, \frac{1}{n}\right)}\right) d t+d V_{t}, \\
& d Q_{t}^{n}=\left(-\frac{1}{2} \frac{Q_{t}^{u}}{\max \left(1-\frac{\left(P_{t}^{u}\right)^{2}+\left(Q_{t}^{u}\right)^{2}}{b}, \frac{1}{n}\right)}\right) d t+d W_{t} .
\end{aligned}
$$

The existence of the strong solution is standard by the Lipschitz property, and the equation for $R_{t}=\left(P_{t}\right)^{2}+\left(Q_{t}\right)^{2}$ is

$$
d R_{t}=\left(-\frac{R_{t}}{1-\frac{R_{t}}{b}}+2\right) d t+2 \sqrt{R_{t}} d B_{t}
$$

Feller's test for explosion shows that $\mathbb{P}\left(\lim \tau_{n}=+\infty\right)=1$. In addition, the $L^{p}$ bound for stress may be obtained:

$$
\mathbb{E}\left(\left|\frac{P_{t}^{u} Q_{t}^{u}}{1-\frac{\left(P_{t}^{u}\right)^{2}+\left(Q_{t}^{u}\right)^{2}}{b}}\right|^{p}\right)^{\frac{1}{p}} \leq c_{1} \exp \left(c_{2} \int_{0}^{1}\left|u_{y}(s)\right|^{2} d s\right) .
$$

In step 2, the well-posedness is established by showing the pathwise uniqueness and by the Yamada-Watanabe theorem, which says that "weak solution + pathwise uniqueness = strong solution" [40].

A standard Galerkin approach is applied in step 3. The a priori estimate is the central problem. For the first energy estimate the result is global:

$$
\frac{1}{2} \frac{d}{d t}\|u\|_{L^{2}}^{2}+\left\|u_{y}\right\|_{L^{2}}^{2}=-\int_{0}^{1} \tau u_{y} d y .
$$

Ito's formula for FENE potential,

$$
\Pi(p, q)=-\frac{b}{2} \ln \left(1-\frac{p^{2}+q^{2}}{b}\right),
$$

is

$$
\begin{aligned}
d \Pi\left(P_{t}^{u}, Q_{t}^{u}\right)= & -\frac{1}{2} \frac{\left(P_{t}^{u}\right)^{2}+\left(Q_{t}^{u}\right)^{2}}{\left(1-\frac{\left(P_{t}^{u}\right)^{2}+\left(Q_{t}^{u}\right)^{2}}{b}\right)^{2}} d t+u_{y} \frac{P_{t}^{u} Q_{t}^{u}}{\left(1-\frac{\left(P_{t}^{u}\right)^{2}+\left(Q_{t}^{u}\right)^{2}}{b}\right)^{2}} d t \\
& +\frac{1}{\left(1-\frac{\left(P_{t}^{u}\right)^{2}+\left(Q_{t}^{u}\right)^{2}}{b}\right)^{2}} d t+\frac{P_{t}^{u}}{\left(1-\frac{\left(P_{t}^{u}\right)^{2}+\left(Q_{t}^{u}\right)^{2}}{b}\right)^{2}} d V_{t} \\
& +\frac{Q_{t}^{u}}{\left(1-\frac{\left(P_{t}^{u}\right)^{2}+\left(Q_{t}^{u}\right)^{2}}{b}\right)^{2}} d W_{t},
\end{aligned}
$$


and hence

$$
\begin{aligned}
& \frac{1}{2} \frac{d}{d t}\|u\|_{L_{y}^{2}}^{2}+\frac{d}{d t} \int_{0}^{1} \mathbb{E} \Pi d y+\left\|u_{y}\right\|_{L_{y}^{2}}^{2}+\frac{1}{2} \int_{0}^{1} \mathbb{E}\left(\frac{\left(P_{t}^{u}\right)^{2}+\left(Q_{t}^{u}\right)^{2}}{\left(1-\frac{\left(P_{t}^{u}\right)^{2}+\left(Q_{t}^{u}\right)^{2}}{b}\right)^{2}}\right) d y \\
& -\int_{0}^{1} \mathbb{E}\left(\frac{1}{\left(1-\frac{\left(P_{t}^{u}\right)^{2}+\left(Q_{t}^{u}\right)^{2}}{b}\right)^{2}}\right) d y=0
\end{aligned}
$$

It can be proved that the fourth term in (3.118) can be bounded above by the third term, and thus the first energy estimate is obtained.

The second energy estimate is as follows:

$$
\frac{d}{d t}\left\|u_{y}\right\|_{L^{2}}^{2}+\left\|u_{y y}\right\|_{L^{2}}^{2} \leq \int_{0}^{1}\left|\tau_{y}\right|\left|u_{y y}\right| d y
$$

Direct calculation shows that

$$
\tau_{y}=\mathbb{E}\left(\frac{P_{t} Q_{t}}{\left(1-\frac{\left(P_{t}\right)^{2}+\left(Q_{t}\right)^{2}}{b}\right)^{2}}\left(\int_{0}^{t} u_{y y} Q_{s} d V_{s}-\int_{0}^{t} u_{y y} u_{y} Q_{s}^{2} d s\right) \mathcal{E}\right) .
$$

Substituting this into (3.119), and taking advantage of the $L^{p}$ estimate for $\mathcal{E}$ and the stress, one will obtain the local second energy estimate. The localness comes from the following exponential type inequality:

$$
R(t) \leq A+C \int_{0}^{t} \exp (\gamma R(s)) d s .
$$

If $t \in\left(0, \frac{1}{\gamma C}(\exp (-\gamma A)-\exp (-2 \gamma A))\right)$, then one has the local bounded result

$$
R(t) \leq 2 A \text {. }
$$

\section{General dumbbell models in high dimensions}

For another extension of the shear flow result, the high dimensional case is considered in [18]. The basic idea for proving the local existence of classical solution is to establish a contraction mapping to the following system:

$$
\begin{aligned}
\partial_{t} \boldsymbol{u}^{n+1}+\left(\boldsymbol{u}^{n} \cdot \nabla_{\boldsymbol{x}}\right) \boldsymbol{u}^{n+1}+\nabla_{\boldsymbol{x}} p^{n+1} & =\Delta_{\boldsymbol{x}} \boldsymbol{u}^{n+1}+\nabla_{\boldsymbol{x}} \cdot \tau^{n}, \\
\nabla_{\boldsymbol{x}} \cdot \boldsymbol{u}^{n+1} & =0 \\
\tau^{n} & =\mathbb{E}\left(\boldsymbol{F}\left(\boldsymbol{Q}^{n}\right) \otimes \boldsymbol{Q}^{n}\right), \\
\partial_{t} \boldsymbol{Q}^{n+1}+\left(\boldsymbol{u}^{n+1} \cdot \nabla_{\boldsymbol{x}}\right) \boldsymbol{Q}^{n+1} & =\kappa^{n+1} \boldsymbol{Q}^{n+1}-\boldsymbol{F}\left(\boldsymbol{Q}^{n+1}\right)+\dot{\boldsymbol{W}}(t) .
\end{aligned}
$$

The basic technique is drawn from the proof of a local classical solution for hyperbolic conservation laws,

$$
\boldsymbol{u}_{t}+\nabla_{\boldsymbol{x}} \cdot \boldsymbol{F}(\boldsymbol{u})=0 .
$$

Since the classical solution satisfies

$$
\frac{\partial \boldsymbol{u}}{\partial t}+\sum_{j=1}^{N} A_{j}(\boldsymbol{u}) \frac{\partial \boldsymbol{u}}{\partial x_{j}}=0
$$


the iteration approach will be:

$$
\frac{\partial \boldsymbol{u}^{k+1}}{\partial t}+\sum_{j=1}^{N} A_{j}\left(\boldsymbol{u}^{k}\right) \frac{\partial \boldsymbol{u}^{k+1}}{\partial x_{j}}=0 .
$$

We need to establish the contraction in the space $H^{m}(\Omega)$, where $m$ is sufficiently high so that it can be embedded into a continuous function space. Kato and Lax's insight to this problem is that this procedure may be simplified by showing

Step 1: boundedness in high norm;

Step 2: contraction in low norm.

By interpolation theory for Sobolev spaces, $\|v\|_{H^{s^{\prime}}} \leq C_{s}\|v\|_{L^{2}}^{1-\frac{s^{\prime}}{s}}\|v\|_{H^{s}}^{\frac{s^{\prime}}{s}}$, we have the inequality

$$
\left\|\boldsymbol{u}^{k}-\boldsymbol{u}^{l}\right\|_{H^{s^{\prime}}} \leq C_{s}\left\|\boldsymbol{u}^{k}-\boldsymbol{u}^{l}\right\|_{L^{2}}^{1-\frac{s^{\prime}}{s}}
$$

for the boundedness in high norm. If $s^{\prime}>\frac{d}{2}, d$ is the spatial dimension, then the Cauchy sequence $\left\{\boldsymbol{u}^{k}\right\}$ in $L^{2}$ will guarantee a Cauchy sequence in $H^{s^{\prime}}$, so the convergence in the continuous function space is proved! Now we are considering the local solution of parabolic type,

$$
\boldsymbol{u}_{t}+\nabla_{\boldsymbol{x}} \cdot \boldsymbol{F}\left(\nabla_{\boldsymbol{x}} \boldsymbol{u}\right)=0
$$

so that a higher order estimate is needed to guarantee the classical solution.

We will only outline the a priori estimates, for the rigorous proof will be folklore. The spatial domain will be assumed in a cube $D$, and the boundary condition is periodic for simplicity. The force $\boldsymbol{F}(\boldsymbol{Q})$ has the form $\gamma\left(Q^{2}\right) \boldsymbol{Q} . \gamma$ is sufficiently smooth, $\gamma^{\prime}\left(Q^{2}\right) \geq 0$, and the derivative of $\boldsymbol{F}$ satisfies $\left|\nabla_{\boldsymbol{Q}}^{m} \boldsymbol{F}(\boldsymbol{Q})\right| \leq C\left(1+|\boldsymbol{Q}|^{p}\right)(m=0,1,2,3,4)$ where $C$ is a constant and $p$ is a certain non-negative integer. Note that this assumption excludes the FENE force.

The a priori estimate for the linearized NSE

$$
\begin{aligned}
\boldsymbol{u}_{t}+\left(\boldsymbol{h} \cdot \nabla_{\boldsymbol{x}}\right) \boldsymbol{u}+\nabla_{\boldsymbol{x}} p & =\Delta_{\boldsymbol{x}} \boldsymbol{u}+\nabla_{\boldsymbol{x}} \cdot \tau, \quad \nabla_{\boldsymbol{x}} \cdot \boldsymbol{u}=0 \\
\nabla_{\boldsymbol{x}} \cdot \boldsymbol{h} & =0
\end{aligned}
$$

is standard. We have for $\alpha=0,1,2,3,4$,

$$
\begin{gathered}
\|\boldsymbol{u}(\cdot, t)\|_{H^{\alpha}}^{2} \leq e^{\int_{0}^{t}\|\boldsymbol{h}(\cdot, s)\|_{H^{4}} d s}\left(\left\|\boldsymbol{u}_{0}\right\|_{H^{\alpha}}^{2}+\int_{0}^{t}\|\tau(\cdot, s)\|_{H^{\alpha}}^{2} d s\right) \\
\int_{0}^{t}\|\boldsymbol{u}(\cdot, s)\|_{H^{\alpha+1}}^{2} d s \leq\left\|\boldsymbol{u}_{0}\right\|_{H^{\alpha}}^{2}+\int_{0}^{t}\left(\|\boldsymbol{h}(\cdot, s)\|_{H^{4}}\|\boldsymbol{u}(\cdot, s)\|_{H^{\alpha}}^{2}+\|\tau(\cdot, s)\|_{H^{\alpha}}^{2}\right) d s .
\end{gathered}
$$

We need to estimate $\|\tau\|_{H^{\alpha}}$ from the equation for $\boldsymbol{Q}$. We have the following estimates by direct calculation:

$$
\begin{aligned}
\|\tau\|_{L^{2}}^{2} & =C \int\left(\mathbb{E}\left(1+|\boldsymbol{Q}|^{p_{1}}\right)\right)^{2} d x \leq C \int\left(1+\mathbb{E}|\boldsymbol{Q}|^{2 p_{1}}\right) d \boldsymbol{x}, \\
\left\|\nabla_{\boldsymbol{x}} \tau\right\|_{L^{2}}^{2} & \leq C \int \mathbb{E}\left(1+|\boldsymbol{Q}|^{2 p_{2}}\right) \mathbb{E}\left|\nabla_{\boldsymbol{x}} \boldsymbol{Q}\right|^{2} d \boldsymbol{x}, \\
\left\|\nabla_{\boldsymbol{x}}^{2} \tau\right\|_{L^{2}}^{2} & \leq C \int \mathbb{E}\left(\left|\nabla_{\boldsymbol{x}} \boldsymbol{Q}\right|^{4}+\left|\nabla_{\boldsymbol{x}}^{2} \boldsymbol{Q}\right|^{2}\right) \mathbb{E}\left(1+|\boldsymbol{Q}|^{2 p_{3}}\right) d \boldsymbol{x}, \\
\left\|\nabla_{\boldsymbol{x}}^{3} \tau\right\|_{L^{2}}^{2} & \leq C \int \mathbb{E}\left(\left|\nabla_{\boldsymbol{x}} \boldsymbol{Q}\right|^{6}+\left|\nabla_{\boldsymbol{x}}^{2} \boldsymbol{Q}\right|^{2}\left|\nabla_{\boldsymbol{x}} \boldsymbol{Q}\right|^{2}+\left|\nabla_{\boldsymbol{x}}^{3} \boldsymbol{Q}\right|^{2}\right) \mathbb{E}\left(1+|\boldsymbol{Q}|^{2 p_{4}}\right) d \boldsymbol{x},
\end{aligned}
$$




$$
\begin{aligned}
\left\|\nabla_{\boldsymbol{x}}^{4} \tau\right\|_{L^{2}}^{2} \leq & C \int \mathbb{E}\left(\left|\nabla_{\boldsymbol{x}}^{4} \boldsymbol{Q}\right|^{2}+\left|\nabla_{\boldsymbol{x}}^{3} \boldsymbol{Q}\right|^{2}\left|\nabla_{\boldsymbol{x}} \boldsymbol{Q}\right|^{2}+\left|\nabla_{\boldsymbol{x}}^{2} \boldsymbol{Q}\right|^{4}+\left|\nabla_{\boldsymbol{x}}^{2} \boldsymbol{Q}\right|^{2}\left|\nabla_{\boldsymbol{x}} \boldsymbol{Q}\right|^{4}\right. \\
& \left.+\left|\nabla_{\boldsymbol{x}} \boldsymbol{Q}\right|^{8}\right) \mathbb{E}\left(1+|\boldsymbol{Q}|^{2 p_{5}}\right) d \boldsymbol{x} .
\end{aligned}
$$

Thus, the $L^{p}$ estimate for $\int_{D} \mathbb{E}\left|\nabla_{\boldsymbol{x}}^{m} \boldsymbol{Q}\right|^{p} d \boldsymbol{x}$ is needed. This may be accomplished by a "pyramid"-type estimate. In fact we prove:

1. $L^{\infty}$-estimate of $\mathbb{E}|\boldsymbol{Q}|^{m}$ for arbitrary $m$;

2. $L^{p}$-estimate of $\int \mathbb{E}\left|\nabla_{\boldsymbol{x}} \boldsymbol{Q}\right|^{p} d \boldsymbol{x}$ for arbitrary $p$;

3. $L^{p}$-estimate of $\int \mathbb{E}\left|\nabla_{\boldsymbol{x}}^{2} \boldsymbol{Q}\right|^{p} d \boldsymbol{x}$ for arbitrary $p \leq 6$;

4. $L^{p}$-estimate of $\int \mathbb{E}\left|\nabla_{\boldsymbol{x}}^{3} \boldsymbol{Q}\right|^{p} d \boldsymbol{x}$ for arbitrary $p \leq 3$;

5. $L^{p}$-estimate of $\int \mathbb{E}\left|\nabla_{\boldsymbol{x}}^{4} \boldsymbol{Q}\right|^{p} d \boldsymbol{x}$ for arbitrary $p \leq 2$.

The proof for the sup $\mathbb{E}|\boldsymbol{Q}|^{m}$ is done by using Lagrangian variables and a standard application of Ito's formula. The higher order derivative estimate is based on the following two points.

1. The leading order of $-\nabla_{\boldsymbol{x}}^{m} \boldsymbol{F}(\boldsymbol{Q})$ is

$$
-\nabla_{\boldsymbol{Q}} \boldsymbol{F} \cdot \nabla_{\boldsymbol{x}}^{m} \boldsymbol{Q}
$$

but

$$
-\left(\nabla_{\boldsymbol{Q}} \boldsymbol{F} \cdot \nabla_{\boldsymbol{x}}^{m} \boldsymbol{Q}\right) * \nabla_{\boldsymbol{x}}^{m} \boldsymbol{Q} \leq 0 .
$$

2. The leading order of the convective term is

$$
\int_{D}\left(\boldsymbol{u} \cdot \nabla_{\boldsymbol{x}}\right) R * R=0, \quad R=\nabla_{\boldsymbol{x}}^{m} \boldsymbol{Q},
$$

by integration by parts. Here $*$ denotes the Frobenius inner product of two tensors.

\section{Rod-like model under shear flow}

The analysis for the stochastic model of LCP is very rare, but it is closely related to interacting particle systems for its mean field physical nature. The basic technique [79] is applied in [49] to prove the well-posedness and numerical analysis for a coupled shear flow system.

The LCP equation under shear flow environment is:

$$
\begin{aligned}
\partial_{t} u & =u_{y y}+\tau_{y}, \\
\mathrm{~d} \Theta_{t}^{u} & =-\left(a\left(\Theta_{t}^{u}, \mathcal{L}\left(\Theta_{t}^{u}\right)\right)+u_{y} \sin ^{2} \Theta_{t}^{u}\right) \mathrm{d} t+\mathrm{d} W_{t}, \\
\tau(y, t) & =\mathbb{E}\left[\sin 2 \Theta_{t}^{u}+a\left(\Theta_{t}^{u}, \mathcal{L}\left(\Theta_{t}^{u}\right)\right) \cos ^{2} \Theta_{t}^{u}+u_{y} \sin ^{2} 2 \Theta_{t}^{u}\right] .
\end{aligned}
$$

Here we have simplified the equations $(2.54)$ and $(2.55)$ by setting $\boldsymbol{m}=(\cos \theta, \sin \theta, 0)$ with Maire-Saupe potential, and

$$
a(\theta, \mu)=\int_{-\infty}^{+\infty} \sin 2\left(\theta-\theta^{\prime}\right) \mu\left(\mathrm{d} \theta^{\prime}\right), \quad \forall \quad \theta \in \mathbb{R}, \mu \in \mathcal{M}
$$

with initial condition $\left.\Theta_{t}^{u}\right|_{t=0}=\Theta_{0}^{u}$. Here $\mathcal{M}$ is the set of all distributions on $\mathbb{R}$.

The main difference between (3.108), (3.109) and the equations here is that the latter form a nonlinear SDE, which means that the realization of each path depends 
on its own distribution: the whole paths' behavior! These kinds of systems have been studied by Kac, McKean, Oelschlager, Sznitman et al. [79, 39, 64, 68]. The propagation of chaos problem is considered in the literature, which is related to gas kinetic theory.

A direct idea to deal with this problem seems to apply the Girsanov transformation to eliminate the $u_{y}$ dependence in the equation for $\Theta_{t}^{u}$. Similar as in [38], but it doesn't work!

Define

$$
\mathrm{d} \Theta_{t}=-a\left(\Theta_{t}, \mathcal{L}\left(\Theta_{t}\right)\right) \mathrm{d} t+\mathrm{d} W_{t},
$$

where $\Theta_{t}$ is defined in $\left(\Omega, \mathcal{F}, \mathcal{F}_{t}, \mathbb{P}\right)$. Define

$$
\bar{W}_{t}=W_{t}-\int_{0}^{t} u_{y} \sin ^{2} \Theta_{s} d s
$$

the new measure $\mathbb{P}^{u}$ and the exponential martingale

$$
\frac{d \mathbb{P}^{u}}{d \mathbb{P}}=\mathcal{E}=\exp \left(\int_{0}^{t} u_{y} \sin ^{2} \Theta_{s} d W_{s}-\frac{1}{2} \int_{0}^{t}\left(u_{y} \sin ^{2} \Theta_{s}\right)^{2} d s\right) .
$$

Then $\bar{W}_{t}$ is a Brownian motion under $\mathbb{P}^{u}$, and formally the SDE becomes

$$
\begin{aligned}
\mathrm{d} \bar{\Theta}_{t} & =\left(-\int_{\Omega} \sin 2\left(\bar{\Theta}_{t}-\Theta_{t}\left(\omega^{\prime}\right)\right) \mathbb{P}\left(d \omega^{\prime}\right)\right) d t+u_{y} \sin ^{2} \bar{\Theta}_{t} d t+d \bar{W}_{t} \\
& =\left(-\int_{\Omega} \sin 2\left(\bar{\Theta}_{t}-\bar{\Theta}_{t}\left(\omega^{\prime}\right)\right) \mathcal{E}^{-1} \mathbb{P}^{u}\left(d \omega^{\prime}\right)\right) d t+u_{y} \sin ^{2} \bar{\Theta}_{t} d t+d \bar{W}_{t} .
\end{aligned}
$$

Thus $u_{y}$ enters the equation for $\bar{\Theta}_{t}$ as Radon-Nikodym derivative, by the nonlinearity of the problem. This issue may be overcome with an idea similar to that for the high dimensional case in the dumbbell model, i.e. to consider the $y$-dependence of $\Theta_{t}$ and applying standard PDE analysis to the coupled system.

The a priori estimates may be accomplished by showing the first and second energy estimates. We will assume that $\Theta_{t}^{u}$ is defined in $\left(\Omega, \mathcal{F}, \mathcal{F}_{t}, \mathbb{P}\right)$ space in the follows.

Step 1. Global in time first energy estimate:

$$
\frac{1}{2} \frac{d}{d t}\|u\|_{L_{y}^{2}}^{2}+\left\|\partial_{y} u\right\|_{L_{y}^{2}}^{2}=-\int_{0}^{1} \partial_{y} \tau u d y,
$$

and we have

$$
\begin{aligned}
\int_{0}^{t} \int_{0}^{1} \partial_{y} \tau u \mathrm{~d} y \mathrm{~d} s & =-\int_{0}^{t} \int_{0}^{1} \tau \partial_{y} u \mathrm{~d} y \mathrm{~d} s \\
& \leq 2 \int_{0}^{t} \int_{0}^{1}\left|\partial_{y} u\right| \mathrm{d} y \mathrm{~d} s-\int_{0}^{t} \int_{0}^{1}\left|\partial_{y} u\right|^{2} \mathbb{E}\left(\sin ^{2} 2 \Theta_{s}^{u}\right) \mathrm{d} y \mathrm{~d} s \\
& \leq \frac{1}{2}\left\|\partial_{y} u\right\|_{L_{t}^{2}\left(L_{y}^{2}\right)}^{2}+C
\end{aligned}
$$

where $C$ depends only on $T$. The proof utilizes the fact that the absolute value of the trigonometric function is less than 1 and the positivity of the last term.

Step 2: Local in time second energy estimate. 
The key issue for the second energy estimate is $\int_{0}^{1} \partial_{y} \tau \partial_{y y} u d y$. If we define $\Phi_{t}=$ $\partial_{y} \Theta_{t}^{u}$, and take advantage of

$$
\tau=\mathbb{E}\left[\sin 2 \Theta_{t}^{u}+\cos ^{2} \Theta_{t}^{u} \int_{\Omega} \sin 2\left(\Theta_{t}^{u}-\Theta_{t}^{u}\left(\omega^{\prime}\right)\right) \mathbb{P}\left(\mathrm{d} \omega^{\prime}\right)+\partial_{y} u \sin ^{2} 2 \Theta_{t}^{u}\right],
$$

the chain rule will give the expression for $\partial_{y} \tau=\partial_{y} \tau\left(\Theta_{t}^{u}, \Phi_{t}, u_{y}, u_{y y}\right)$. Moreover, the equation (3.134) for $\Theta_{t}$ gives:

$$
\begin{array}{r}
\mathrm{d} \Phi_{t}=-\left(\int_{\Omega} 2 \cos 2\left(\Theta_{t}^{u}-\Theta_{t}^{u}\left(\omega^{\prime}\right)\right)\left(\Phi_{t}-\Phi_{t}\left(\omega^{\prime}\right)\right) \mathrm{d} \mathbb{P}\left(\omega^{\prime}\right)\right. \\
\left.+\partial_{y y} u \sin ^{2} \Theta_{t}^{u}+\partial_{y} u \Phi_{t} \sin 2 \Theta_{t}^{u}\right) \mathrm{d} t .
\end{array}
$$

The Ito formula and Gronwall's inequality show that

$$
\left\|\Phi_{t}\right\|_{L_{y}^{2}\left(L_{\omega}^{2}\right)}^{2} \leq\left(\left\|\Phi_{0}\right\|_{L_{y}^{2}\left(L_{\omega}^{2}\right)}^{2}+4 \int_{0}^{t}\left\|\partial_{y y} u\right\|_{L_{y}^{2}}^{2} \mathrm{~d} s\right) \exp \left(4 \int_{0}^{t}\left(1+\left\|\partial_{y} u\right\|_{L_{y}^{\infty}}\right) \mathrm{d} s\right),
$$

where $\Phi_{0}$ is the initial value of $\Phi_{t}$. Substituting the estimate to the inequality of $\left\|\partial_{y} \tau\right\|_{L_{y}^{2}}^{2}$, one obtains the following estimate, similar to the one in [38]

$$
\|u\|_{L_{t}^{\infty}\left(\left[0, T^{\prime}\right], H_{y}^{1}\right)}+\|u\|_{L_{t}^{2}\left(\left[0, T^{\prime}\right], H_{y}^{2}\right)} \leq C, \quad \text { for } t \in\left(0, T^{\prime}\right)
$$

where $T^{\prime}$ depends only on $\left\|u_{0}\right\|_{H_{y}^{1}}, T$, and $\left\|\Phi_{0}\right\|_{L_{y}^{2} L_{\omega}^{2}}$.

\section{Numerical analysis of the models}

Numerical algorithms directly based on the kinetic theory for complex fluids have been developed since the 1970s. The simulations are quite computationally demanding in general, thus they will only become available after the developments of faster computers. For the deterministic version of micro models, one needs to solve a very high dimensional PDEs not only in position space $\boldsymbol{x}$ but also in configuration space $\boldsymbol{Q}$ or $\boldsymbol{n}$. At present, it is generally not feasible in three or higher dimensions. As another candidate, stochastic simulations have the virtue of being dimension-independent and parallel in nature, which is a very promising approach for multi-scale simulations of complex configurations (for example a polymer chain). Both procedures are currently computationally challenging. In order to simplify the problem further, closure approximations are made to form a deterministic PDEs for polymeric stress. They are very common in the chemical engineering community. Their use in numerical simulations of complex fluids is reviewed by Owens and Phillips [74] and Keunings [41]. The micro-macro numerical techniques, which include mathematical formulations and numerical methods of viscoelastic fluids, are reviewed by Keunings [42]. In this paper we will focus on the convergence analysis for multiscale models.

The stochastic (or Brownian dynamics) simulations for kinetic models of polymeric liquids have been applied to various models [72]. Laso and Öttinger seem to be the first to design the so-called CONNFFESSIT ( $\underline{\text { Calculation } \underline{\text { Of }} \text { Non-Newtonian }}$ Flow: Finite Elements and $\underline{S}$ tochastic SImulation Technique) [45]. In this method, a collection of $N$ dumbbells spread at each element are evolved independently according to (2.9) and (2.10). The polymeric stress is then calculated by ensemble averaging over the dumbbells inside each element. CONNFFESSIT is a Lagrangian method that follows the trajectories of the dumbbells. As such, it suffers from standard problems associated with Lagrangian methods, e.g. the local concentration and sparsity 
of dumbbells in different elements, which will deteriorate the accuracy of the stress. To overcome these difficulties, Hulsen et. al introduced the Brownian configuration fields (BCF) method [31, 73] based on the dynamics of configuration fields. Now the dumbbells are modeled by $N$ fields $\boldsymbol{Q}_{i}(\boldsymbol{x}, t), i=1, \ldots, N$. $\boldsymbol{Q}_{i}$ evolves independently according to (2.25), and the extra stress is again computed through ensemble averaging over the $N$ fields at each grid point. This Eulerian approach eliminates the local concentration problem and also reduces the noise in the results. It is further deeply analyzed and extended in [35].

For stochastic simulations, variance reduction is a central issue because the mean square error of Monte Carlo methods is related to the variance through

$$
\mathbb{E}\left(\langle X\rangle-\langle X\rangle_{N}\right)^{2}=\frac{\operatorname{Var}(X)}{N}
$$

for any random variable $X$, where $\operatorname{Var}(\mathrm{X})$ is the variance of $X$. Even if the time stepsize $\Delta t$ is very small and the sampling size $N$ is very large, the large variance will deteriorate the computational results. Some variance reduction ideas and techniques have been considered for polymeric fluids such as $[65,73,6,35,47,66]$ etc.. Special structures of the problems are utilized for different cases. Melchior et al. [65, 66] proposed a number of variance reduction methods for general SDEs based on importance sampling strategies and the idea of control variables. BCF and the Lagrangian particle method (LPM) of Halin et al. [30] are examples of variances reduced simulations based on the idea of correlated local ensembles of polymers. This idea is further developed in [6, 35]. T. Li et al. [47] consider the numerical issues for small Deborah number simulations. They apply the asymptotic analysis for the SDEs and the HMM idea[16] to overcome time scale issues. The zero Deborah number limit is obtained. More work on variance reduction is needed in the future.

For the deterministic simulation of kinetic models, Warner solved the FokkerPlanck equation under the condition of steady-state shearing flow and small amplitude oscillatory shearing flow of a FENE fluid in 1972 [81]. Fan [19, 20] improved the original idea of Warner by requiring that the probability density function be smooth at the origin, leading to more accurate results. Lozinski et al. [58, 59] introduced some fast spectral methods for the Fokker-Planck equations for some models of diluted and concentrated polymer solutions. The start-up of steady shear flow for diluted solutions of rod-like macromolecules was also treated with the Fokker-Planck equation using wavelets for the discretization in configuration space by Nayak [67]. $\mathrm{Yu}$ et. al. [83] proposed a finite-difference scheme that exploits the probabilistic nature of the equation by means of a discrete analog of the Chapman-Kolmogorov equation, so that the numerical solutions preserve the positivity and the unity of the probability distribution function. Spherical harmonics [45, 21] were chosen because an expansion in this basis cannot only describe the equilibrium spherical distribution function exactly, but also describe very accurately the small deviations from the equilibrium distribution that are produced by lower shear rate flow. The finite element method on the sphere [46] was developed based on spherical geodesic grids to solve the Fokker-Planck equation of rod-like model. The Fokker-Planck-based methods can be significantly more efficient than their stochastic counterparts for models in lower-dimensional configurations space.

Although the Fokker-Planck equations are analytically equivalent to SDEs, the numerical methods and numerical analysis based on these equations are very different. In this section, we will focus on the numerical analysis of stochastic and deterministic methods for some simple micro-macro models of polymeric fluids. 
4.1. Stochastic approach for the coupled micro-macro model. As the stochastic simulations become increasingly popular, interest in the numerical analysis of these methods has grown. The analysis for the Hookean dumbbell model in the shear flow is performed in [17] and [37] independently. It is generalized to the high dimensional Hookean dumbbell model in [49] and the rodlike model in shear flow [48]. The analysis for nonlinear dumbbells is still under investigation.

\section{Hookean dumbbell model under shear flow}

A standard time discretization and Monte Carlo discretization for (3.95)-(3.98) is as follows:

$$
\begin{aligned}
u^{n+1}-u^{n} & =u_{y y}^{n+1} \Delta t+\partial_{y}\left(\left\langle P^{n} Q^{n}\right\rangle_{N}\right) \Delta t, \\
P_{i}^{n+1} & =P_{i}^{n}+\left(u_{y}^{n+1} Q_{i}^{n}-\frac{P_{i}^{n}}{2}\right) \Delta t+d V_{i}^{n}, \\
Q_{i}^{n+1} & =Q_{i}^{n}-\frac{Q_{i}^{n}}{2} \Delta t+d W_{i}^{n},
\end{aligned}
$$

where $d V_{i}^{n}, d W_{i}^{n}$ are i.i.d. $N(0, \Delta t)$ random variables, and $i=1,2, \ldots, N$. Here and in the following we use $\langle\cdot\rangle_{N}$ to denote empirical averages, i.e.

$$
\left\langle P^{n} Q^{n}\right\rangle_{N} \triangleq \frac{1}{N} \sum_{i=1}^{N} P_{i}^{n} Q_{i}^{n} .
$$

Consider the truncated equation:

$$
u\left(t_{n+1}\right)-u\left(t_{n}\right)=u_{y y}\left(t_{n+1}\right) \Delta t+\partial_{y}\left\langle P_{t} Q_{t}\right\rangle\left(t_{n}\right) \Delta t+O\left(\Delta t^{2}\right) .
$$

Then we have the error equation

$$
e^{n+1}=e^{n}+\Delta t e_{y y}^{n+1}+\Delta t \partial_{y}\left(\left\langle P^{n} Q^{n}\right\rangle_{N}-\left\langle P_{t} Q_{t}\right\rangle\left(t_{n}\right)\right)+O\left(\Delta t^{2}\right)
$$

and a simple energy estimate shows that

$$
\begin{gathered}
\frac{1}{2}\left(\left\|e^{n+1}\right\|_{L_{y}^{2}}^{2}-\left\|e^{n}\right\|_{L_{y}^{2}}^{2}\right)+\Delta t\left\|e_{y}^{n+1}\right\|_{L_{y}^{2}}^{2} \leq \\
\Delta t \int_{0}^{1} \partial_{y}\left(\left\langle P^{n} Q^{n}\right\rangle_{N}-\left\langle P_{t} Q_{t}\right\rangle\left(t_{n}\right)\right) e^{n+1} d y+\Delta t\left\|e^{n+1}\right\|_{L_{y}^{2}}^{2}+O\left(\Delta t^{3}\right) .
\end{gathered}
$$

Let us introduce the auxiliary equation:

$$
\begin{aligned}
& \tilde{P}^{n+1}=\tilde{P}^{n}+\left(u_{y}\left(t_{n+1}\right) \tilde{Q}^{n}-\frac{\tilde{P}^{n}}{2}\right) \Delta t+d V^{n}, \\
& \tilde{Q}^{n+1}=\tilde{Q}^{n}-\frac{\tilde{Q}^{n}}{2} \Delta t+d W^{n},
\end{aligned}
$$

and its $n$-replica,

$$
\begin{aligned}
& \hat{P}_{i}^{n+1}=\hat{P}_{i}^{n}+\left(u_{y}\left(t_{n+1}\right) \hat{Q}_{i}^{n}-\frac{\hat{P}_{i}^{n}}{2}\right) \Delta t+d V_{i}^{n}, \\
& \hat{Q}_{i}^{n+1}=\hat{Q}_{i}^{n}-\frac{\hat{Q}_{i}^{n}}{2} \Delta t+d W_{i}^{n},
\end{aligned}
$$


where $d V_{i}^{n}, d W_{i}^{n}$ are the same as in equation (4.3) and (4.4).

Then we have

$$
\begin{aligned}
\left\langle P_{t} Q_{t}\right\rangle\left(t_{n}\right)-\left\langle P^{n} Q^{n}\right\rangle_{N} \leq & \left(\left\langle P_{t} Q_{t}\right\rangle\left(t_{n}\right)-\left\langle\tilde{P}^{n} \tilde{Q}^{n}\right\rangle\right)+\left(\left\langle\tilde{P}^{n} \tilde{Q}^{n}\right\rangle-\left\langle\hat{P}^{n} \hat{Q}^{n}\right\rangle_{N}\right) \\
& +\left(\left\langle\hat{P}^{n} \hat{Q}^{n}\right\rangle_{N}-\left\langle P^{n} Q^{n}\right\rangle_{N}\right) \\
= & E_{1}+E_{2}+E_{3},
\end{aligned}
$$

where $E_{1}$ is the time discretization error, $E_{2}$ is the sampling error, and $E_{3}$ is the discretization error induced by the velocity $u$.

It is not difficult to show that

$$
E_{1} \sim O(\Delta t), \quad \mathbb{E} E_{2} \sim O\left(\frac{1}{\sqrt{N}}\right)
$$

by a standard numerical SDE result[43] and the Law of Large Numbers.

For the $E_{3}$ term, we have

$$
\begin{aligned}
\int_{0}^{1}\left(\left\langle\hat{P}^{n} \hat{Q}^{n}\right\rangle_{N}-\left\langle P^{n} Q^{n}\right\rangle_{N}\right) e_{y}^{n+1} d y & =\int_{0}^{1}\left(\left\langle\left(\hat{P}^{n}-P^{n}\right) \hat{Q}^{n}\right\rangle_{N}\right) e_{y}^{n+1} d y \\
& =\int_{0}^{1}\left\langle E_{P}^{n} \hat{Q}^{n}\right\rangle_{N} e_{y}^{n+1} d y
\end{aligned}
$$

For the error of the equation for $P$, we have

$$
\begin{aligned}
E_{P, i}^{n+1} & =E_{P, i}^{n}+\Delta t\left(e_{y}^{n+1} \hat{Q}_{i}^{n}-\frac{E_{P, i}^{n}}{2}\right) \\
& =\left(1-\frac{\Delta t}{2}\right) E_{P, i}^{n}+\Delta t e_{y}^{n+1} \hat{Q}_{i}^{n} .
\end{aligned}
$$

Hence, squaring both sides we obtain

$$
\left(E_{P, i}^{n+1}\right)^{2} \leq\left(E_{P, i}^{n}\right)^{2}+2 \Delta t e_{y}^{n+1} \hat{Q}_{i}^{n} E_{P, i}^{n}+\frac{3}{2} \Delta t^{2}\left(e_{y}^{n+1}\right)^{2}\left(\hat{Q}_{i}^{n}\right)^{2}-\frac{1}{2} \Delta t\left(E_{P, i}^{n}\right)^{2} .
$$

Taking summation, we have

$$
\begin{aligned}
\left\langle\left\|E_{P}^{n+1}\right\|_{L_{y}^{2}}^{2}\right\rangle_{N} & +\left\|e^{n+1}\right\|_{L_{y}^{2}}^{2}+\Delta t\left\|e_{y}^{n+1}\right\|_{L_{y}^{2}}^{2} \leq(1+C \Delta t)\left(\left\langle\left\|E_{P}^{n}\right\|_{L_{y}^{2}}^{2}\right\rangle_{N}+\left\|e^{n}\right\|_{L_{y}^{2}}^{2}\right) \\
& +\Delta t^{2}\left\|e_{y}^{n+1}\right\|_{L_{y}^{2}}^{2}\left\langle\left(\hat{Q}^{n}\right)^{2}\right\rangle_{N}+C \delta t\left(\left\langle\left\|E_{3}\right\|_{L_{y}^{2}}^{2}\right\rangle_{N}+\Delta t^{2}\right) .
\end{aligned}
$$

Here $e_{y}^{n+1}$ and $\left\langle\left(\hat{Q}^{n}\right)^{2}\right\rangle_{N}$ are correlated random variables. Though $\mathbb{E}\left\langle\left(\hat{Q}^{n}\right)^{2}\right\rangle_{N}$ is bounded, the expectation cannot be taken inside! But the large deviation estimate guarantees $\Delta t\left\langle\left(\hat{Q}^{n}\right)^{2}\right\rangle_{N} \leq \frac{1}{2}$ for all $n$ after excluding a set of exponentially small measure. That gives the convergence of the type contained in [18],

$$
\left\|e^{n}\right\|_{L_{y}^{2}}^{2} \leq \Delta t^{2}+\frac{\xi}{N} \quad \text { in } \mathcal{A}^{c},
$$

where $\mathbb{E}|\xi| \leq$ Const., or in [37],

$$
\mathbb{E}\left(\left\|e^{n}\right\|_{L_{y}^{2}}^{2} \cdot \mathbf{1}_{\mathcal{A}^{c}}\right) \leq \Delta t^{2}+\frac{1}{N} .
$$


Here, $\mathcal{A}$ is a set of exponentially small probability, and $\mathcal{A}^{c}$ is its complement.

\section{Rod-like model under shear flow}

The numerical discretization of the equation (3.134) of $\Theta_{t}^{u}$ will be more interesting; we utilize the basic technique in [79]. For simplicity, we only introduce the estimate in the absence of flow. Now the equation is (3.137), and its discretization is

$$
\Theta_{t}^{j}=\Theta_{0}-\int_{0}^{t} \frac{1}{M} \sum_{k=1}^{M} \sin 2\left(\Theta_{s}^{j}-\Theta_{s}^{k}\right) \mathrm{d} s+W_{t}^{j}, \quad j=1,2, \cdots, M,
$$

in analogy to the Monte-Carlo method. Notice here that all of the $\Theta_{t}^{j}$ are coupled and the Monte-Carlo discretization $\frac{1}{M} \sum_{k=1}^{M} \sin 2\left(\Theta_{s}^{j}-\Theta_{s}^{k}\right)$ does not have the traditional meaning, but

$$
\frac{1}{M} \sum_{k=1}^{M} \sin 2\left(\Theta_{t}^{j}-\Theta_{t}^{k}\right) \stackrel{M \rightarrow \infty}{\longrightarrow} \int_{-\infty}^{+\infty} \sin 2\left(\Theta_{t}^{j}-\Theta^{\prime}\right) \mathcal{L}_{\Theta_{t}^{j}}\left(d \Theta^{\prime}\right)
$$

which means the correlation among $\Theta_{t}^{j}(j=1, \ldots, M)$ will disappear as $M \rightarrow \infty$. This is closely related to the so called "propagation of chaos" concept proposed by M. Kac. We consider the weaker form

$$
\left\|\mathbb{E} \sin 2 \Theta_{t}-\frac{1}{M} \sum_{j=1}^{M} \sin 2 \Theta_{t}^{j}\right\|_{L_{\omega}^{2}}^{2} \leq \frac{C}{M} .
$$

Let us introduce $M$ i.i.d. samples of $\Theta_{t}$

$$
\tilde{\Theta}_{t}^{j}=\Theta_{0}-\int_{0}^{t} a\left(\tilde{\Theta}_{s}^{j}, \mathcal{L}\left(\tilde{\Theta}_{s}^{j}\right)\right) \mathrm{d} s+W_{t}^{j}, \quad j=1,2, \cdots, M .
$$

Then

$$
\begin{aligned}
\left\|\mathbb{E} \sin 2 \Theta_{t}-\frac{1}{M} \sum_{j=1}^{M} \sin 2 \Theta_{t}^{j}\right\|_{L_{\omega}^{2}}^{2} \leq & \left\|\mathbb{E} \sin 2 \Theta_{t}-\frac{1}{M} \sum_{j=1}^{M} \sin 2 \tilde{\Theta}_{t}^{j}\right\|_{L_{\omega}^{2}}^{2} \\
& +\left\|\frac{1}{M} \sum_{j=1}^{M} \sin 2 \tilde{\Theta}_{t}^{j}-\frac{1}{M} \sum_{j=1}^{M} \sin 2 \Theta_{t}^{j}\right\|_{L_{\omega}^{2}}^{2},
\end{aligned}
$$

and by (4.17) and (4.20)

$$
\begin{aligned}
\left|\Theta_{t}^{j}-\tilde{\Theta}_{t}^{j}\right|^{2} & =\left|\int_{0}^{t} \frac{1}{M} \sum_{k=1}^{M} \sin 2\left(\Theta_{s}^{j}-\Theta_{s}^{k}\right)-a\left(\tilde{\Theta}_{s}^{j}, \mathcal{L}\left(\tilde{\Theta}_{s}^{j}\right)\right) \mathrm{d} s\right|^{2} \\
& \leq 2 t \int_{0}^{t}\left|\frac{1}{M} \sum_{k=1}^{M} \sin 2\left(\Theta_{s}^{j}-\Theta_{s}^{k}\right)-a\left(\tilde{\Theta}_{s}^{j}, \mathcal{L}\left(\Theta_{s}^{j}\right)\right)\right|^{2} d s .
\end{aligned}
$$

We have

$$
\begin{aligned}
\sin 2\left(\Theta_{t}^{j}-\Theta_{t}^{k}\right)-a\left(\tilde{\Theta}_{t}^{j}, \mathcal{L}\left(\tilde{\Theta}_{t}^{j}\right)\right)= & \left(\sin 2\left(\Theta_{t}^{j}-\Theta_{t}^{k}\right)-\sin 2\left(\tilde{\Theta}_{t}^{j}-\Theta_{t}^{k}\right)\right) \\
& +\left(\sin 2\left(\tilde{\Theta}_{t}^{j}-\Theta_{t}^{k}\right)-\sin 2\left(\tilde{\Theta}_{t}^{j}-\tilde{\Theta}_{t}^{k}\right)\right)+b_{j k}
\end{aligned}
$$


in which $b_{j k}=\sin 2\left(\tilde{\Theta}_{t}^{j}-\tilde{\Theta}_{t}^{k}\right)-a\left(\tilde{\Theta}_{t}^{j}, \mathcal{L}\left(\tilde{\Theta}_{t}^{j}\right)\right)$.

The Law of Large Numbers implies that

$$
\begin{aligned}
& \mathbb{E}\left[b_{j k} b_{j l}\right] \\
= & \mathbb{E}_{\left(\tilde{\Theta}_{t}^{j}, \tilde{\Theta}_{t}^{k}, \tilde{\Theta}_{t}^{l}\right)}\left[\left(\sin 2\left(\tilde{\Theta}_{t}^{j}-\tilde{\Theta}_{t}^{k}\right)-a\left(\tilde{\Theta}_{t}^{j}, \mathcal{L}\left(\tilde{\Theta}_{t}^{j}\right)\right)\right) .\right. \\
& \left.\cdot\left(\sin 2\left(\tilde{\Theta}_{t}^{j}-\tilde{\Theta}_{t}^{l}\right)-a\left(\tilde{\Theta}_{t}^{j}, \mathcal{L}\left(\tilde{\Theta}_{t}^{j}\right)\right)\right)\right] \\
= & \mathbb{E}_{\tilde{\Theta}_{t}^{j}}\left[\mathbb{E}_{\tilde{\Theta}_{t}^{k}}\left(\sin 2\left(\tilde{\Theta}_{t}^{j}-\tilde{\Theta}_{t}^{k}\right)-a\left(\tilde{\Theta}_{t}^{j}, \mathcal{L}\left(\tilde{\Theta}_{t}^{j}\right)\right)\right) \cdot\right. \\
& \left.\cdot \mathbb{E}_{\tilde{\Theta}_{t}^{l}}\left(\sin 2\left(\tilde{\Theta}_{t}^{j}-\tilde{\Theta}_{t}^{l}\right)-a\left(\tilde{\Theta}_{t}^{j}, \mathcal{L}\left(\tilde{\Theta}_{t}^{j}\right)\right)\right)\right] \\
= & \mathbb{E}_{\tilde{\Theta}_{t}^{j}}\left[\left(a\left(\tilde{\Theta}_{t}^{j}, \mathcal{L}\left(\tilde{\Theta}_{t}^{k}\right)\right)-a\left(\tilde{\Theta}_{t}^{j}, \mathcal{L}\left(\tilde{\Theta}_{t}^{j}\right)\right)\right)\left(a\left(\tilde{\Theta}_{t}^{j}, \mathcal{L}\left(\tilde{\Theta}_{t}^{l}\right)\right)-a\left(\tilde{\Theta}_{t}^{j}, \mathcal{L}\left(\tilde{\Theta}_{t}^{j}\right)\right)\right)\right] \\
= & \mathbb{E}_{\tilde{\Theta}_{t}^{j}}[0]=0 .
\end{aligned}
$$

The Lipschitz property of the sine function will give the desired estimate!

\section{High dimensional Hookean dumbbell model}

The convergence analysis of the BCF method is generalized to the high dimensional Hookean dumbbell model in [49]. The basic approach is very similar to the shear flow case presented before. But a stronger result is obtained for the analysis of the error of the polymeric stress $\langle\boldsymbol{Q} \otimes \boldsymbol{Q}\rangle$.

The first point in the proof is that the space should be discretized. In this case the inverse inequality

$$
\left\|\boldsymbol{u}^{n}\right\|_{L_{h}^{\infty}} \leq h^{-\frac{d}{2}}\left\|\boldsymbol{u}^{n}\right\|_{L_{h}^{2}}, \quad\left\|\tilde{\boldsymbol{Q}}^{n}\right\|_{L_{h}^{\infty}} \leq h^{-\frac{d}{2}}\left\|\tilde{\boldsymbol{Q}}^{n}\right\|_{L_{h}^{2}}
$$

can be applied, where $\tilde{\boldsymbol{Q}}^{n}$ is the numerical solution of equation (2.25) and $\boldsymbol{u}\left(t_{n}\right)$ denotes the exact solution. Let us consider the error of $\boldsymbol{Q}$,

$$
\boldsymbol{E}^{n}:=\boldsymbol{Q}^{n}-\hat{\boldsymbol{Q}}^{n},
$$

only with time discretization. Here the notations $\boldsymbol{Q}^{n}, \tilde{\boldsymbol{Q}}^{n}$ and $\hat{\boldsymbol{Q}}^{n}$ are similar to those in the shear flow case. $\boldsymbol{E}^{n}$ satisfies

$$
\frac{1}{\Delta t}\left(\boldsymbol{E}^{n+1}-\boldsymbol{E}^{n}\right)+\boldsymbol{u}^{n} \cdot \nabla \boldsymbol{E}^{n+1}+\boldsymbol{e}^{n} \cdot \nabla \hat{\boldsymbol{Q}}^{n+1}=\kappa^{n} \boldsymbol{E}^{n+1}+\nabla \boldsymbol{e}^{n} \hat{\boldsymbol{Q}}^{n+1}-\boldsymbol{E}^{n} .
$$

The term

$$
\int_{D} \boldsymbol{u}^{n} \cdot \nabla \boldsymbol{E}^{n+1} \cdot \boldsymbol{E}^{n+1} d \boldsymbol{x}=0
$$

similar to the continuous case. In order to estimate $\int_{D} e^{n} \cdot \nabla \hat{\boldsymbol{Q}}^{n+1} \cdot \boldsymbol{E}^{n+1} d \boldsymbol{x}$, one needs

$$
\left\|\left\langle\left|\nabla \hat{\boldsymbol{Q}}^{n+1}\right|^{2}\right\rangle_{N}\right\|_{L^{\infty}} \preceq \text { Const. }
$$

where " $\preceq$ " means " $\leq$ " after excluding a set of exponentially small probability, however this could be very difficult! It is easier to obtain the following $L^{2}$-type estimate,

$$
\left\|\left\langle\left|\nabla \hat{\boldsymbol{Q}}^{n+1}\right|^{2}\right\rangle_{N}\right\|_{L^{2}} \preceq \text { Const. }
$$


But it cannot be transfered back to the $L^{\infty}$ norm. This difficulty could be overcome by using the inverse inequality trick shown above in the spatially discrete case.

Even if the space is discretized, the inverse inequality makes the estimate of the type in [37] inapplicable. Now the term $\boldsymbol{u}^{n} \cdot \nabla \boldsymbol{E}^{n+1}$ becomes $\boldsymbol{u}^{n} \cdot \nabla_{h} \boldsymbol{E}^{n+1}$, and in this discrete case,

$$
\sum_{i j} \boldsymbol{u}^{n} \cdot \nabla_{h} \boldsymbol{E}^{n+1} \cdot \boldsymbol{E}^{n+1} \neq 0
$$

It only has the estimate

$$
\left|\sum_{i j} \boldsymbol{u}^{n} \cdot \nabla_{h} \boldsymbol{E}^{n+1} \cdot \boldsymbol{E}^{n+1}\right| \leq\left\|\nabla_{h} \boldsymbol{u}^{n}\right\|_{L_{h}^{\infty}}\left\|\boldsymbol{E}^{n+1}\right\|_{L_{h}^{2}}
$$

One demands

$$
\Delta t\left\|\nabla_{h} \boldsymbol{u}^{n}\right\|_{L_{h}^{\infty}} \preceq \text { Const. }
$$

This could be transferred to the $L_{h}^{2}$ estimate by an inverse inequality argument, but a convergence result of the type in [37] only gives

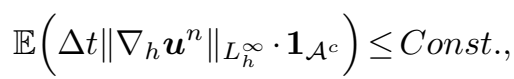

where $\mathcal{A}$ is a set of exponentially small probability. The expectation cannot be eliminated, which means a stronger result is needed.

The key point in [49] is to prove

$$
\left\|\left\langle\tilde{\boldsymbol{Q}}^{n} \otimes \tilde{\boldsymbol{Q}}^{n}\right\rangle-\left\langle\hat{\boldsymbol{Q}}^{n} \otimes \hat{\boldsymbol{Q}}^{n}\right\rangle_{N}\right\|_{L_{h}^{\infty}}^{2} \preceq \frac{1}{N^{1-\epsilon}},
$$

where $\epsilon$ is an arbitrarily small positive real number. This goal is achieved by observing that $\boldsymbol{Q}$ is a Gaussian process and the discrete $\hat{\boldsymbol{Q}}^{n}$ are i.i.d. Gaussian random variables.

Define the flow map

$$
\frac{d \boldsymbol{x}(\boldsymbol{\alpha}, t)}{d t}=\boldsymbol{u}(\boldsymbol{x}(\boldsymbol{\alpha}, t), t), \quad \boldsymbol{x}(\boldsymbol{\alpha}, 0)=\boldsymbol{\alpha}
$$

and let $\boldsymbol{Q}(\boldsymbol{\alpha}, t)=\boldsymbol{Q}(\boldsymbol{x}(\boldsymbol{\alpha}, t), t)$. Define the deformation tensor

$$
F(\boldsymbol{\alpha}, t)=\frac{\partial \boldsymbol{x}}{\partial \boldsymbol{\alpha}}, \text { i.e. } \quad F_{i j}=\frac{\partial x_{i}}{\partial \alpha_{j}},
$$

Then we can obtain the explicit solution

$$
\boldsymbol{Q}(\boldsymbol{x}, t)=e^{-t} F(\boldsymbol{x}, t) \boldsymbol{Q}_{0}(\boldsymbol{x})+F(\boldsymbol{x}, t) \cdot \int_{0}^{t} e^{s-t} F^{-1}(\boldsymbol{x}, s) \cdot d \boldsymbol{W}_{s} .
$$

If $\boldsymbol{Q}_{0}(\boldsymbol{x})$ is a spatially smooth Gaussian random field, then $\boldsymbol{Q}(\boldsymbol{x}, t)$ is a spatially smooth Gaussian process. A similar result holds for discrete $\tilde{\boldsymbol{Q}}^{n}$.

Based upon this observation, the excluded exponentially small sets may be clarified without ambiguity. Because the large deviation rate function for the random variables $\left(\tilde{Q}^{k}\right)^{2}$ and $\tilde{Q}^{k} \tilde{Q}^{l}(k \neq l)$ can be explicitly calculated, thus the inequality (4.33) can be obtained. The inverse inequality trick ensures a second order convergence result of the type in [57]. 
4.2. Deterministic approach for the coupled micro-macro model.

Solving directly the Fokker-Planck equation for the probability density instead of the stochastic differential equation can alleviate the three main disadvantages of the stochastic method, which are large CPU cost, huge memory requirements and the presence of statistical noise in the computed extra stress.

The spectral and finite element methods do not guarantee the positivity of the probability density function, which is often important for stability of numerical scheme, especially for numerical analysis. Du et al. [83, 11] presented a finite difference scheme by means of a discrete analog of the Chapman-Kolmogorov equation which has the positivity-preserving and the unity-preserving features of FENE type models. Zhang et al. [85] designed a positivity-preserving scheme for a rod-like model coupling shear flow and proved the convergence using the positivity-preserving property.

In this subsection, we want to show the convergence of a fully discretized scheme for equations (2.20), (2.21), (2.53) and (2.54) under the shear flow condition,

$$
\left\{\begin{array}{l}
u_{t}+c=\frac{\gamma}{R e} u_{y y}+\frac{1-\gamma}{D e R e} \tau_{y}, \\
\psi_{t}=\frac{1}{D e} \psi_{\theta \theta}+\frac{1}{D e}\left(\psi U_{\theta}\right)_{\theta}+u_{y}\left(\psi \sin ^{2} \theta\right)_{\theta}, \\
U=U_{0} \int_{0}^{2 \pi} \sin ^{2}\left(\theta-\theta^{\prime}\right) \psi\left(y, \theta^{\prime}, t\right) d \theta^{\prime}, \\
\tau=2\langle\sin \theta \cos \theta\rangle+\left\langle U_{\theta} \cos ^{2} \theta\right\rangle+\frac{D e}{2} u_{y} \cdot\left\langle\sin ^{2} \theta \cos ^{2} \theta\right\rangle,
\end{array}\right.
$$

with the backward Euler method in the temporal direction and the center difference method in the spatial ones, respectively. The discrete velocity is defined on a uniform mesh $\mathcal{T}_{h}$, where $h=1 / N$ is the space discretization step. The time interval $(0, T)$ is discretized with a uniform step size $\Delta t$. We will define the discrete functions $\bar{u}_{j+1 / 2}^{n}$ and $\bar{\psi}_{j, k}^{n}$ at $((j+1 / 2) h, n \triangle t)$ and $(j h, k \sigma, n \triangle t)$, respectively. We also let $y_{j}=j h, \theta_{k}=$ $k \sigma$, where $\sigma=2 \pi / M$ is the discretization configuration space step.

The problem (4.35) can be discretized in the following form:

$$
\begin{aligned}
\frac{\bar{u}_{j+1 / 2}^{n+1}-\bar{u}_{j+1 / 2}^{n}=}{\Delta t}= & \frac{\gamma}{R e} \frac{\left(D_{h} \bar{u}\right)_{j+1}^{n+1}-\left(D_{h} \bar{u}\right)_{j}^{n+1}}{h}+\frac{1-\gamma}{R e D e} \frac{\left(\bar{\tau}_{\alpha}\right)_{j+1}^{n}-\left(\bar{\tau}_{\alpha}\right)_{j}^{n}}{h} \\
& +\frac{1-\gamma}{2 R e} \frac{\left(D_{h} \bar{u}\right)_{j+1}^{n+1}\left(\bar{\tau}_{\beta}\right)_{j+1}^{n}-\left(D_{h} \bar{u}\right)_{j}^{n+1}\left(\bar{\tau}_{\beta}\right)_{j}^{n}}{h}-c \\
\frac{\bar{\psi}_{j, k}^{n+1}-\bar{\psi}_{j, k}^{n}}{\Delta t}= & \frac{1}{D e} \frac{\left(D_{\sigma} \bar{\psi}\right)_{j, k+1 / 2}^{n+1}-\left(D_{\sigma} \bar{\psi}\right)_{j, k-1 / 2}^{n+1}}{\sigma} \\
& +\frac{1}{D e} \frac{\left(\bar{\psi} \bar{U}_{\theta}\right)_{j, k+1 / 2}^{n+1}-\left(\bar{\psi} \bar{U}_{\theta}\right)_{j, k-1 / 2}^{n+1}}{\sigma} \\
& +\left(D_{h} \bar{u}\right)_{j}^{n} \frac{\left(\bar{\psi} \sin ^{2} \theta\right)_{j, k+1 / 2}^{n+1}-\left(\bar{\psi} \sin ^{2} \theta\right)_{j, k-1 / 2}^{n+1}}{\sigma}
\end{aligned}
$$

with initial and boundary conditions:

$$
\begin{aligned}
\bar{u}_{j+1 / 2}^{0} & =u_{0}((j+1 / 2) h), \quad j=0, \cdots, N-1 ; \\
\bar{\psi}_{j, k}^{0} & =\frac{1}{\sigma} \int_{(k-1 / 2) \sigma}^{(k+1 / 2) \sigma} \psi_{0}(j h, \theta) d \theta, j=0, \cdots, N ; \quad k=0, \cdots, M-1, \\
\bar{u}_{-1 / 2}^{n} & =-\bar{u}_{1 / 2}^{n}, \quad \bar{u}_{N+1 / 2}^{n}=-\bar{u}_{N-1 / 2}^{n}, \quad n=1,2, \cdots,
\end{aligned}
$$


where

$$
\begin{aligned}
\left(\bar{\tau}_{\alpha}\right)_{j}^{n} & =\sum_{k=0}^{M-1}\left(\sin \left(2 \theta_{k}\right)+\left(\bar{U}_{\theta}\right)_{j, k}^{n} \cos ^{2} \theta_{k}\right) \bar{\psi}_{j, k}^{n} \sigma \\
\left(\bar{\tau}_{\beta}\right)_{j}^{n} & =\sum_{k=0}^{M-1} \sin ^{2} \theta_{k} \cos ^{2} \theta_{k} \bar{\psi}_{j, k}^{n} \sigma \\
\left(\bar{U}_{\theta}\right)_{j, k}^{n} & =U_{0}\left[\sin 2 \theta_{k} \sum_{l=0}^{M-1} \cos 2 \theta_{l} \bar{\psi}_{j, l}^{n-1} \sigma-\cos 2 \theta_{k} \sum_{l=0}^{M-1} \sin 2 \theta_{l} \bar{\psi}_{j, l}^{n-1} \sigma\right] \\
\left(D_{h} \bar{u}\right)_{j}^{n} & =\frac{\bar{u}_{j+1 / 2}^{n}-\bar{u}_{j-1 / 2}^{n}}{h}, \quad\left(D_{\sigma} \bar{\psi}\right)_{j, k+1 / 2}^{n}=\frac{\bar{\psi}_{j, k+1}^{n}-\bar{\psi}_{j, k}^{n}}{\sigma} \\
\left(\bar{\psi} \sin ^{2} \theta\right)_{j, k+1 / 2}^{n}= & \frac{\left(\bar{\psi} \sin ^{2} \theta\right)_{j, k+1}^{n}+\left(\bar{\psi} \sin ^{2} \theta\right)_{j, k}^{n}}{2} .
\end{aligned}
$$

It follows from (4.37) that

$$
\sigma \sum_{l=0}^{M-1} \psi_{j, l}^{n}=1, \quad \text { for all } n \in \mathbb{N}
$$

provided that $\sigma \sum_{l=0}^{M-1} \psi_{j, l}^{0}=1$.

We define $u_{j+1 / 2}^{n}=u((j+1 / 2) h, n \Delta t), \psi_{j, k}^{n}=\psi(j h, k \sigma, n \Delta t)$ and define $\left(\tau_{\alpha}\right)_{j}^{n}$, $\left(\tau_{\beta}\right)_{j}^{n},\left(U_{\theta}\right)_{j, k}^{n}$ similarly. It is easy to verify that $u_{j+1 / 2}^{n}, \psi_{j, k}^{n}$ satisfy the difference equations (4.36) and (4.37) with the truncation errors $\mathcal{O}\left(\Delta t+h^{2}+\sigma^{2}\right)$. The numerical boundary condition (4.40) is also of second order accuracy in space.

The generic constant $C$ is assumed to be independent of the mesh size $h, \sigma$ and $\Delta t$. Suppose $\left\|u_{y}\right\|_{\infty} \leq C_{1}$ and define

$$
T^{*}=\sup \left\{t_{1} \in[0, T] ;\left\|D_{h} \bar{u}^{n}\right\|_{l^{\infty}} \leq C_{1}+1, n \Delta t \in\left[0, t_{1}\right]\right\}
$$

Then there exists an $\sigma_{0}$ such that

$$
\bar{\psi}_{j, k}^{n} \geq 0, \quad \sigma<\sigma_{0}, \quad n \Delta t<T^{*}
$$

by using the property of the $M$-matrix. The non-negativity property of the probability distribution and (4.46) yield

$$
\begin{aligned}
\left(\bar{\tau}_{\beta}\right)_{j}^{n} & \geq 0, \\
\left\|\bar{\tau}_{\alpha}^{n}\right\|_{l^{\infty}} & \leq C, \quad\left\|\tau_{\alpha}^{n}\right\|_{l^{\infty}} \leq C, \\
\left\|\bar{U}_{\theta}^{n}\right\|_{l^{\infty}} & \leq C .
\end{aligned}
$$

Set

$$
\begin{aligned}
e_{j+1 / 2}^{n} & =\bar{u}_{j+1 / 2}^{n}-u_{j+1 / 2}^{n}, \\
E_{j, k}^{n} & =\bar{\psi}_{j, k}^{n}-\psi_{j, k}^{n} .
\end{aligned}
$$


We then have

$$
\begin{aligned}
\frac{e_{j+1 / 2}^{n+1}-e_{j+1 / 2}^{n}=}{\Delta t}= & \frac{\gamma}{R e} \frac{\left(D_{h} e\right)_{j+1}^{n+1}-\left(D_{h} e\right)_{j}^{n+1}}{h} \\
& +\frac{1-\gamma}{R e D e} \frac{\left(\bar{\tau}_{\alpha}-\tau_{\alpha}\right)_{j+1}^{n}-\left(\bar{\tau}_{\alpha}-\tau_{\alpha}\right)_{j}^{n}}{h} \\
& +\frac{1-\gamma}{2 R e} \frac{\left(D_{h} e\right)_{j+1}^{n+1}\left(\bar{\tau}_{\beta}\right)_{j+1}^{n}-\left(D_{h} e\right)_{j}^{n+1}\left(\bar{\tau}_{\beta}\right)_{j}^{n}}{h} \\
& +\frac{1-\gamma}{2 R e} \frac{\left(D_{h} u\right)_{j+1}^{n+1}\left(\bar{\tau}_{\beta}-\tau_{\beta}\right)_{j+1}^{n}-\left(D_{h} u\right)_{j}^{n+1}\left(\bar{\tau}_{\beta}-\tau_{\beta}\right)_{j}^{n}}{h} \\
& +C\left(\Delta t+h^{2}+\sigma^{2}\right),
\end{aligned}
$$

and

$$
\begin{aligned}
\frac{E_{j, k}^{n+1}-E_{j, k}^{n}=}{\Delta t}= & \frac{1}{D e} \frac{\left(D_{\sigma} E\right)_{j, k+1 / 2}^{n+1}-\left(D_{\sigma} E\right)_{j, k-1 / 2}^{n+1}}{\sigma} \\
& +\frac{1}{D e} \frac{\left(E \bar{U}_{\theta}\right)_{j, k+1 / 2}^{n+1}-\left(E \bar{U}_{\theta}\right)_{j, k-1 / 2}^{n+1}}{\sigma} \\
& +\frac{1}{D e} \frac{\left(\psi\left(\bar{U}_{\theta}-U_{\theta}\right)\right)_{j, k+1 / 2}^{n+1}-\left(\psi\left(\bar{U}_{\theta}-U_{\theta}\right)\right)_{j, k-1 / 2}^{n+1}}{\sigma} \\
& +\left(D_{h} e\right)_{j}^{n+1} \frac{\left(\psi \sin ^{2} \theta\right)_{j, k+1 / 2}^{n+1}-\left(\psi \sin ^{2} \theta\right)_{j, k-1 / 2}^{n+1}}{\sigma} \\
& +\left(D_{h} \bar{u}\right)_{j}^{n+1} \frac{\left(E \sin ^{2} \theta\right)_{j, k+1 / 2}^{n+1}-\left(E \sin ^{2} \theta\right)_{j, k-1 / 2}^{n+1}}{\sigma} \\
& +C\left(\Delta t+h^{2}+\sigma^{2}\right) .
\end{aligned}
$$

Multiplying (4.54) with $e_{j+1 / 2}^{n+1}$ and summing the resulting equation with respect to $j$ yields

$$
\begin{gathered}
\frac{h}{2 \Delta t} \sum_{j=0}^{N-1}\left|e_{j+1 / 2}^{n+1}\right|^{2}+\frac{\gamma h}{2 R e} \sum_{j=0}^{N-1}\left|D_{h} e_{j}^{n+1}\right|^{2} \\
\leq \frac{h}{2 \Delta t} \sum_{j=0}^{N-1}\left|e_{j+1 / 2}^{n}\right|^{2}+C\left\|\bar{\tau}_{\alpha}^{n}-\tau_{\alpha}^{n}\right\|_{l^{2}([0,1])}^{2} \\
\quad+C\left\|\bar{\tau}_{\beta}^{n}-\tau_{\beta}^{n}\right\|_{l^{2}([0,1])}^{2}+C\left(\Delta t+h^{2}+\sigma^{2}\right)^{2} .
\end{gathered}
$$

Similarly, we obtain by using (4.54) that

$$
\begin{aligned}
& \frac{h \sigma}{2 \Delta t} \sum_{j, k}\left|E_{j, k}^{n+1}\right|^{2}+\frac{h \sigma}{2 D e} \sum_{j, k}\left|D_{\sigma} E_{j, k+1 / 2}^{n+1}\right|^{2} \\
\leq & \frac{h \sigma}{2 \Delta t} \sum_{j, k}\left|E_{j, k}^{n}\right|^{2}+C h \sum_{j=0}^{N-1}\left|D_{h} e_{j}^{n+1}\right|^{2}+C h \sigma \sum_{j, k}\left|E_{j, k}^{n+1}\right|^{2} \\
& +C\left\|\bar{U}_{\theta}^{n+1}-U_{\theta}^{n+1}\right\|_{l^{2}([0,1] \times[0,2 \pi])}^{2}+C\left(\Delta t+h^{2}+\sigma^{2}\right)^{2} .
\end{aligned}
$$


It is easy to verify that

$$
\begin{aligned}
\left\|\bar{\tau}_{\alpha}^{n}-\tau_{\alpha}^{n}\right\|_{l^{2}([0,1])}^{2} & \leq C h \sigma \sum_{j, k}\left|E_{j, k}^{n}\right|^{2} \\
\left\|\bar{\tau}_{\beta}^{n}-\tau_{\beta}^{n}\right\|_{l^{2}([0,1])}^{2} & \leq C h \sigma \sum_{j, k}\left|E_{j, k}^{n}\right|^{2} \\
\left\|\bar{U}_{\theta}^{n+1}-U_{\theta}^{n+1}\right\|_{l^{2}([0,1] \times[0,2 \pi])}^{2} & \leq C h \sigma \sum_{j, k}\left|E_{j, k}^{n+1}\right|^{2} .
\end{aligned}
$$

Combining (4.54)-(4.59) gives

$$
\begin{aligned}
& h \sum_{j=0}^{N-1}\left|e_{j+1 / 2}^{n+1}\right|^{2}+h \sigma \sum_{j, k}\left|E_{j, k}^{n+1}\right|^{2} \\
\leq & (1+C \Delta t)\left(h \sum_{j=0}^{N-1}\left|e_{j+1 / 2}^{n}\right|^{2}+h \sigma \sum_{j, k}\left|E_{j, k}^{n}\right|^{2}\right)+C\left(\Delta t+h^{2}+\sigma^{2}\right)^{2} .
\end{aligned}
$$

The above Gronwall-type inequality leads to

$$
h \sum_{j=0}^{N-1}\left|e_{j+1 / 2}^{n}\right|^{2}+h \sigma \sum_{j, k}\left|E_{j, k}^{n}\right|^{2} \leq C\left(\Delta t+h^{2}+\sigma^{2}\right)^{2} .
$$

\section{Conclusions}

As the enormous progress was made in computer technology and computational methods during the last few decades, computer simulations have become a very powerful and widely used tool in polymer science. Modelling and understanding the flow behavior of polymeric fluids on the kinetic level is not merely a great intellectual challenge but rather a matter of immense practical importance in plastics manufacture, processing of foods, and movement of biological fluids, etc.

The multiscale methods of complex fluids that couple the mesoscopic scale with the macroscopic scale of continuum mechanics have an important role to play. On the one hand, this approach is much more demanding in computing resources than conventional continuum simulations. On the other hand, multiscale techniques allow the direct use of kinetic models and thus avoid potentially inexact closure approximations. In most of the cases, the derivation of a macroscopic constitutive equation from kinetic theory involves closure approximations, such as decoupling or pre-averaging, etc. This difficult theoretical issue is bypassed when micro-macro models are used.

Multiscale modelling in polymeric fluids is a very active and rapidly expanding research area. We expect this field to continue to move forward rapidly, particularly with the advances in the mathematical analysis and numerical analysis of multiscale models that we highlighted in this review. As computing power increases and more efficient algorithms are developed, we will have the ability to simulate more realistic models, eventually to simulate industrial processing flows and to get more accurate predictions of the product properties.

Acknowledgement. The authors are very grateful to Professors Weinan E, Qiang Du, Chun Liu, Qi Wang and Hui Zhang for their helpful discussions. T. Li is partially supported by National Science Foundation of China 10401004 and the National Basic Research Program under grant 2005CB321704. P. Zhang is partially 
supported by the special funds for Major State Research Projects 2005CB321704 and National Science Foundation of China for Distinguished Young Scholars 10225103 and 20490222.

\section{REFERENCES}

[1] M. P. Allen and D. J. Tildesley, Computer Simulation of Liquids, Clarendon Press, Oxford, 1987.

[2] J. Barrett, C. Schwab and E. Süli, Existence of global weak solution for some polymeric flow models, Math. Models Methods Appl. Sci., 15, 939-983, 2005.

[3] J. Barrett and E. Süli, Existence of global weak solutions to kinetic models for dilute polymers, submitted to Multi. Model. Simul., 2006.

[4] R. B. Bird, O. Hassager, R. C. Armstrong and C. F. Curtiss, Dynamics of Polymeric Liquids; Vol. 1: Fluid Mechanics, 2nd edition, Wiley-Interscience, New York, 1987.

[5] R. B. Bird, O. Hassager, R. C. Armstrong and C. F. Curtiss, Dynamics of Polymeric Liquids; Vol. 2: Kinetic Theory, 2nd edition, Wiley-Interscience, New York, 1987.

[6] J. Bovin and M. Picasso, Variance reduction methods for CONFFESSIT-like simulations, J. Non-Newtonian Fluid Mech., 84, 191-215, 1999.

[7] P. Constantin, Nonlinear Fokker-Planck Navier-Stokes systems, Comm. Math. Sci., 3, 531-544, 2005.

[8] P. Constantin, I. Kevrekidis and E. S. Titi, Asymptotic states of a Smoluchowski equation, Arch. Rat. Mech. Anal., 174, 365-384, 2004.

[9] P. Constantin, I. Kevrekidis and E. S. Titi, Remarks on a Smoluchowski equation, Discrete Contin. Dynam. Sys., 11, 101-112, 2004.

[10] P. Constantin and J. Vukadinovic, Note on the number of steady states for a 2D Smoluchowski equation, Nonlinearity, 18, 441-443, 2005.

[11] Q. Du, C. Liu and P. Yu, FENE dumbbell model and its several linear and nonlinear closure approximations, Multi. Model. Simul., 4, 709-731, 2005.

[12] P. G. de Gennes, Scaling Concepts in Polymer Physics, Cornell University Press, Ithaca, 1979.

[13] P. G. de Gennes and J. Prost, The Physics of Liquid Crystal, Clarendon Press, Oxford, 1993.

[14] M. Doi and S. F. Edwards, The Theory of Polymer Dynamics, Oxford University Press, Oxford, 1986.

[15] M. Doi, Introduction to Polymer Physics, Clarendon Press, Oxford, 1996.

[16] W. E and B. Engquist, The heterogeneous multiscale methods, Comm. Math. Sci., 1, 87-132, 2003.

[17] W. E, T. Li and P. Zhang, Convergence of a stochastic method for the modeling of polymeric fluids, Acta Mathematicae Applicatae Sinica, English Series, 18, 529-536, 2002.

[18] W. E, T. Li and P. Zhang, Well-posedness of dumbbell model for the polymeric liquids, Comm. Math. Phys., 248, 409-427, 2004.

[19] X. J. Fan, Viscosity, first normal stress coefficient, and molecular stretching in dilute polymer solutions, J. Non-Newtonian Fluid Mech., 17, 125-144, 1985.

[20] X. J. Fan, Viscosity, first normal stress coefficient, and molecular stretching in concentrated solutions and melts, J. Non-Newtonian Fluid Mech., 17, 251-265, 1985.

[21] V. Faraoni, M. Grosso, S. Crescitelli and P. L. Maffettone, The rigid-rod model for nematic polymers: an analysis of the shear flow problem, J. Rheol., 43, 829-843, 1999.

[22] I. Fatkullin and V. Slastikov, A note on the Onsager model of nematic phase transitions, Comm. Math. Sci., 3, 21-26, 2005.

[23] I. Fatkullin and V. Slastikov, Critical points of the Onsager functional on a sphere, Nonlinearity, 18, 2565-2580, 2005.

[24] J. D. Ferry, Viscoelastic Properties of Polymers, 3rd edition, Wiley, New York, 1980.

[25] P. J. Flory, Principles of Polymer Chemistry, Cornell University Press, Ithaca, NY, 1953.

[26] P. J. Flory, The Statistical Mechanics of Chain Molecules, Interscience, New York, 1969.

[27] D. Frenkel and B. Smit, Understanding Molecular Simulation: From Algorithms to Applications, 2nd edition, Academic Press, San Diego, 2002.

[28] M. G. Forest, R. Zhou and Q. Wang, Full-tensor alignment criteria for sheared nematic polymers, J. Rheol., 47, 105-127, 2003.

[29] C. Guillope and J. C. Saut, Existence results for the flow of viscoelastic fluids with a differnetial costitutive law, Nonlinear Anal., 15, 849-869, 1990.

[30] P. Halin, G. Lielens, R. Keunings and V. Legat, The Lagrangian particle method for macroscopic and micro-macro viscoelastic flow computations, J. Non-Newtonian Fluid Mech., 79, 387-403, 1998. 
[31] M. A. Hulsen, A. P. G. van Heel and B. H. A. A. van den Brule, Simulation of viscoelastic flows using Brownian configuration fields, J. Non-Newtonian Fluid Mech., 70, 79-101, 1997.

[32] E. P. Hsu, Stochastic Analysis on Manifolds, American Mathematical Society, Providence, Rhode Island, 2002.

[33] R. Jordan, D. Kinderlehrer and F. Otto, The variational formulation of the Fokker-Planck equation, SIAM J. Math. Anal., 29, 1-17, 1998.

[34] B. Jourdain and T. Lelièvre, Convergence of a stochastic particle approximation of the stress tensor for the FENE-P model, CERMICS, 2004-263, report.

[35] B. Jourdain, C. Le Bris and T. Lelièvre, On a variance reduction technique for the micro-macro simulations of polymeric fluids, J. Non-Newtonian Fluid Mech., 122, 91-106, 2004.

[36] B. Jourdain, C. Le Bris and T. Lelièvre, Coupling PDEs and SDEs: the illustrative example of the multiscale simulation of viscoelasic flows, in Multiscale Methods in Science and Engineering, B. Engquist, P. Lötstedt, O. Runborg (Eds.), CSE, Springer, 44, 151-170, 2005.

[37] B. Jourdain, T. Lelièvre and C. Le Bris, Numerical analysis of micro-macro simulations of polymeric fluid flows: a simple case, Math. Models Methods Appl. Sci., 12, 1205-1243, 2002.

[38] B. Jourdain, T. Lelièvre and C. Le Bris, Existence of solution for a micro-macro model of polymeric fluid: the FENE model, J. Funct. Anal., 209, 162-193, 2004.

[39] M. Kac, Foundation of kinetic theory, Proc. Third Berkeley Symp. On Math. Stat. and Probab., Univ. of Calif. Press, 3, 171-197, 1956.

[40] I. Karatzas and S. E. Shreve, Brownian Motion and Stochastic Calculus, 2nd edition, SpringerVerlag, New York.

[41] R. Keunings, Finite element methods for integral viscoelastic fluids, in Rheology Reviews 2003, D. M. Binding and K. Walters (Eds.), British Society of Rheology, Aberystwyth, Wales, 167-195, 2003.

[42] R. Keunings, Micro-macro methods for the multiscale simulation of viscoelastic flow using molecular models of kenitic theory, in Rheology Reviews 2004, 67-98, D. M. Binding and K. Walters (Eds.), British Society of Rheology, Aberystwyth, Wales, 2004.

[43] P. E. Kloeden and E. Platen, Numerical Solution of Stochastic Differential Equations, SpringerVerlag, Berlin and New York, 1995.

[44] R. G. Larson, The Structure and Reology of Complex Fluids, Oxford University Press, Oxford, 1999.

[45] M. Laso and H. C. Öttinger, Calculation of viscoelastic flow using molecular models: the CONNFFESSIT approach, J. Non-Newtonian Fluid Mech., 47, 1-20, 1993.

[46] R. Li, C. Luo and P. Zhang, Numerical simulation of Doi model of polymeric fluids, Advances in Scientific Computing and Applications, Y. Lu, W. Sun and T. Tang (Eds.), 258-273, 2003.

[47] T. Li, E. Vanden-Eijnden, P. Zhang and W. E, Stochastic models of polymeric fluids at small Deborah number, J. Non-Newtonian Fluid Mech., 121, 117-125, 2004.

[48] T. Li, H. Zhang and P. Zhang, Local existence for the dumbbell model of polymeric fluids, Comm. PDE, 29, 903-923, 2004.

[49] T. Li and P. Zhang, Convergence analysis of the BCF methods for high dimensional Hookean dumbbell model, Multi. Model. Simul., 5, 205-234, 2006.

[50] T. Li, P. Zhang and X. Zhou, Analysis of $1+1$ dimensional stochastic models of liquid crystal polymer flows, Comm. Math. Sci., 2, 295-316, 2004.

[51] F. Lin and C. Liu, Existence of solutions for the Ericksen-Leslie system, Arch. Rat. Mech. Anal., 154, 135-156, 2000.

[52] F. Lin, C. Liu and P. Zhang, On hydrodynamics of viscoelastic fluids, Comm. Pure Appl. Math., 58, 1437-1471, 2005.

[53] F. Lin, C. Liu and P. Zhang, On a micro-macro model for polymeric fluids near equilibrium, Comm. Pure Appl. Math., 2007.

[54] P. L. Lions and N. Masmoudi, Global solutions for some Oldroyd models of non-Newtonian flows, Chin. Ann. of Math., 21B, 131-146, 2000.

[55] C. Liu and H. Liu, Boundary conditions for the microscopic FENE models, SIAM J. Appl. Math., submitted, 2006.

[56] H. Liu, H. Zhang and P. Zhang, Axial symmetry and classification of stationary solutions of Doi-Onsager equation on the sphere with Maier-Saupe potential, Comm. Math. Sci., 3, 201-218, 2005.

[57] D.-G. Long, Convergence of the random vortex method in two dimensions, J. Amer. Math. Soc., 1, 779-804, 1988.

[58] A. Lozinski and C. Chauviere, A fast solver for Fokker-Planck equation applied to viscoelastic 
flows calculations: 2D FENE model, J. Comp. Phys., 189, 607-625, 2003.

[59] A. Lozinski, C. Chauviere, J. Fan and R. G. Owens, A Fokker-Planck simulation of fast flows of melts and concentracted polymer solutions in complex solutions in complex geometries, J. Rheol., 47, 535-561, 2003.

[60] C. Luo, H. Zhang and P. W. Zhang, The structure of equilibrium solutions of the onedimensional Doi equation, Nonlinearity, 18, 379-389, 2005.

[61] P. L. Maffettone and S. Crescitelli, Bifurcation analysis of a molecular model for nematic polymers in shear flows, J. Non-Newtonian Fluid Mech., 59, 73-91, 1995.

[62] W. Maier and A. Z. Saupe, A simple molecular-statistics theory of the nematic liquid-crystalline state, Z. Naturforsch, 13a, 564-570, 1958; A simple molecular-statistics theory of the nematic liquid-crystalline phase, part I, 14a, 882-900, 1959; A simple molecular-statistics theory of the nematic liquid-crystalline phase, part II, 15a, 287-292, 1960.

[63] G. Marrucci and P. L. Maffettone, Description of the liquid crystalline phase of rodlike polymers at high shear rates, Macromolecules, 22, 4076-4082, 1989.

[64] H. P. McKean, Propagation of chaos for a class of nonlinear parabolic equations, Lecture series in differential equations, Catholic University, Washington D.C., 7, 41-57, 1967.

[65] M. Melchior and H. C. Öttinger, Variance reduced simulations of stochastic differential equations, J. Chem. Phys., 103, 9506-9509, 1995.

[66] M. Melchior and H. C. Öttinger, Variance reduced simulations of polymer dynamics, J. Chem. Phys., 105, 3316-3331, 1996.

[67] R. Nayak, Molecular simulation of liquid crystal polymer flow: a wavelet finite element analysis, Ph.D thesis, MIT, 1998.

[68] K. Oelschlager, A martingale approach to the Law of Large Numbers for weakly interacting stochastic processes, Ann. Prob., 12, 458-479, 1984.

[69] B. Oksendal, Stochastic Differential Equations: An Introduction with Applications, 5th edition, Springer-Verlag, Berlin and New York, 1998.

[70] L. Onsager, The effects of shape on the interaction of colloidal particles, Ann. N.Y. Acad. Sci., 51, 627-659, 1949.

[71] A. Onuki, Phase Transition Dynamcis, Cambridge University Press, Cambridge, 2002.

[72] H.C. Öttinger, Stochastic Processes in Polymeric Liquids, Springer-Verlag, Berlin and New York, 1996.

[73] H. C. Öttinger, B. H. A. A. van den Brule and M. A. Hulsen, Brownian configuration fields and variance reduced CONFFESSIT, J. Non-Newtonian Fluid Mech., 70, 255-261, 1997.

[74] R. G. Owens and T. N. Phillips, Computational Rheology, Imperial College Press, London 2002.

[75] M. Renardy, Local existence of solutions of the dirichlet initial-boundary value problem for incompressible hypoelastic materials, SIAM J. Math. Anal., 21, 1369-1385, 1990.

[76] M. Renardy, An existence theorem for model equations resulting from kinetic theories of polymer solutions, SIAM J. Math. Anal., 22, 313-327, 1991.

[77] F. Schmid, Self-consistent-field theories for complex fluids, J. Phys.: Condens. Matter, 10, 8105-8138, 1998.

[78] J. K. C. Suen, Y. L. Joo and R. C. Armstrong, Molecular orientation effects in viscoelasticity, Ann. Rev. Fluid Mech., 34, 417-444, 2002.

[79] A. S. Sznitman, Topics in propogation of chaos, S. Albeverio ed., Pitman Research Notes in Mathematics, 124, 167-251, 1985.

[80] H. Tanaka, Viscoelastic phase separation, J. Phys.: Condens. Matter, 12, 207-264, 2000.

[81] H. R. Warner, Kinetic theory and rheology of dilute suspensions of finitely extensible dumbbells, Ind. Eng. Chem. Fundam., 11, 379-387, 1972.

[82] Qi Wang, A hydrodynamic theory for solutions of nonhomogeneous nematic liquid crystalline polymers of different configurations, J. Chem. Phys., 116, 9120-9136, 2002.

[83] P. Yu, Q. Du and C. Liu, From micro to macro dynamics via a new closure approximation to the FENE model of polymeric fluids, Multi. Model. Simul., 3, 895-917, 2005.

[84] H. Zhang and P. Zhang, A theoretical and numerical study for the rod-like model of polymeric fluid, J. Comp. Math., 22, 319-330, 2004.

[85] H. Zhang and P. Zhang, Local existence for the FENE dumbbell model of polymeric fluids, Arch. Rat. Mech. Anal., 181, 373-400, 2006.

[86] H. Zhang and P. Zhang, Global existence of the solution to the rod-like model of polymeric fluid, preprint, 2004.

[87] H. Zhou, Y. Zhang and Z. Ouyang, Bending and base-stacking interactions in double-stranded DNA, Phys. Rev. Lett., 82, 4560-4563, 1999.

[88] H. Zhou, H. Wang, M. Forest and Q. Wang, A new proof on axisymmetric equilibria of a three-dimensional Smoluchowski equation, Nonlinearity, 18, 2815-2825, 2005. 